\title{
A MEASUREMENT OF $\pi^{\circ}$ PRODUCTION * AT LOW TRANSVERSE MOMENTUM
}

\author{
$\mathrm{By}$ \\ Giuseppe Ballocehi \\ Submitticd \\ in Partial Fullfillment \\ of the \\ Requirements for the Degree of

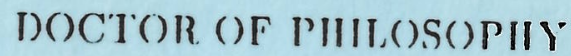

\author{
Supervised by \\ Prof. Thomas Ferbel \\ Department of Physics and Astronomy \\ University of Rochester \\ Ruchester, New York \\ 1989
}

*Work supported by the U.S. Department of Energy Contract No. DE-AC02-76ER13065. 
$\Lambda$ e povr Luigin ed Baloch (1887-1979) e a la povra Disola ed Bunun (1889-1988) 


\section{VITAE}

\section{The author}

attended the University of Bologna where he received a Laurea in Physics in Dccember 1982. During 1982-1983, the author was associated with the INFN in Bologna.

He undertook graduate studies in Physics at the University of Rochester in September 1983. During his studies at the University of Rochester, the author received support from a rescarch assistantship and a Rush Rhees fellowship. The work presented in this thesis was carried out under the supervision of Professor Thomas Ferbel. 


\section{ACKNOWIEDGMENTS}

I wish to thank all the individuals who made this experiment possible. The list of collaborators is too long to be reported here; I would like to thank in particular all the people in the collaboration for many stimulating discussions. In particular I acknowledge the advice I received from Carl Bromberg, George Ginther, Phil Gutierrez, Joey IIuston, Fred Lobkowicz, Paul Slaltery and Marek Zielinski.

This research was supported in part by the U.S. Department of Energy and the National Science Foundation.

I wish to thank my advisor, Professor Thomas Ferbel for his help and advice. I would also like to thank Mrs. Betty Cook for her help in dealing with university matters throughout my graduate career. I have benefitted from discussions with many theorists, in particular Rolf Baier, Patrick Aurençhe, Ed Berger, Roberto Odorico, Jeff Owens, Frank Paige and Tjobiorn Sjostrand. 


\title{
ABSTRAC'T
}

\section{A MEASUREMENT OF $\pi^{0}$ PRODUCTION AT LOW TRANSVERSE MOMENTUM}

\author{
By
}

\section{Giuseppe Ballocchi}

This thesis describes the measurement of $\pi^{0}$ production at low transverse momentum $\left(p_{T}\right)$ in $530 \mathrm{GeV}$ pion-nucleon collisions (center of mass energy of 31.7 $\mathrm{GeV}$ ), utilizing the E706 spectrometer at Fermilab. Electromagnetic showers were detected using a large liquid argon calorimetcr, and charged tracks were measured in a charged particle spectrometer, which included a silicon strip system for vertex identification, a magnet and multiwire proportional chambers.

A measurent of the differential cross section for $\pi^{0}$ production in the $p_{T}$ range $0.6-2.2 \mathrm{GeV} / \mathrm{c}$ is given for Be and $\mathrm{Al}$ targets. The atomic dependence of the measurement is also discussed. 


\section{Contents}

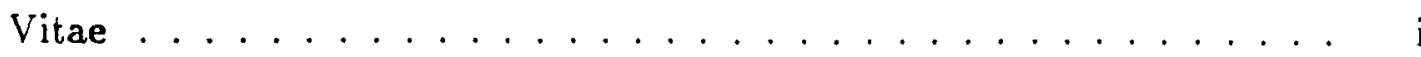

Aknowledgements ................ ii

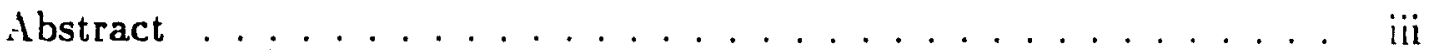

List of Tables . . . . . . . . . . . . . . . . . t

List of Figures . . . . . . . . . . . . . . . .

$\begin{array}{llr}1 & \text { Introduction } & 9\end{array}$

1.1 General considerations . . . . . . . . . . . . . . . 9

1.2 Theoretical models . . . . . . . . . . . . . . . . 10

1.3 Kinematics variables $\ldots \ldots \ldots \ldots \ldots$

1.4 Scaling and limiting fragmentation . . . . . . . . 12

1.5 Experimental measurements of low $p_{T}$ neutral pion produc-

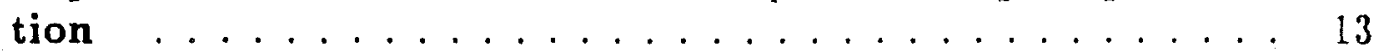

1.6 Atomic dependence of inclusive hadronic cross sections . . 16

2 Experimental apparatus $\quad 17$

2.1 General considerations . . . . . . . . . . . . . 17

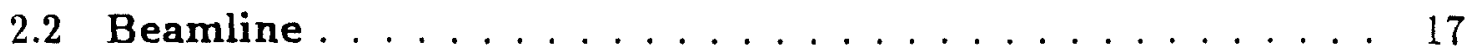

2.3 Targets ......................... 19

2.4 Charged particle detection $\ldots \ldots \ldots \ldots \ldots$

2.5 Liquid argon calorimeter . . . . . . . . . . . . . . . . 20

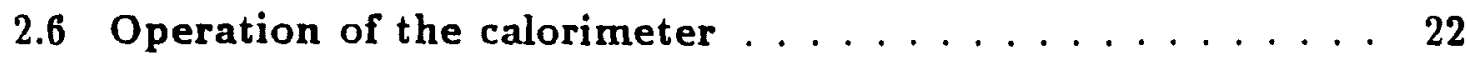

2.7 Calorimeter readout electronics $\ldots \ldots \ldots \ldots \ldots$ 


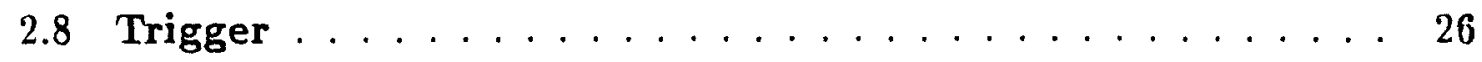

2.9 Forward calorimeter $\ldots \ldots \ldots \ldots \ldots \ldots$

2.10 On-line monitoring and data acquisition $\ldots \ldots \ldots 31$

3 Event reconstruction 32

3.1 General considerations . . . . . . . . . . . . . 32

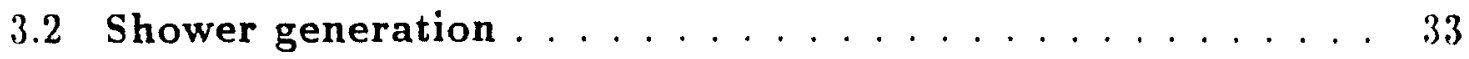

3.3 Shower reconstruction $\ldots \ldots \ldots \ldots \ldots \ldots$

3.4 Groups and peaks . . . . . . . . . . . . . 36

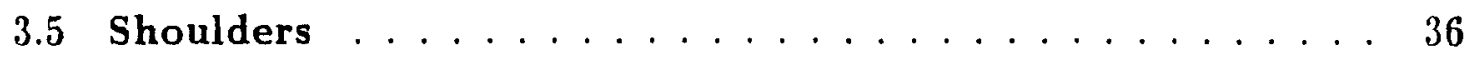

3.6 Splitting of peaks $\ldots \ldots \ldots \ldots \ldots \ldots \ldots$

3.7 Correlation of $\mathbf{r}$ and $\phi$ views $\ldots \ldots \ldots \ldots$

3.8 Prediction of $\mathbf{r}$ from the width of the shower in $\phi \ldots \ldots 41$

3.9 Walker mass $\ldots \ldots \ldots \ldots \ldots \ldots$

3.10 Testing the reconstructor using Monte Carlo events . . . . . 18

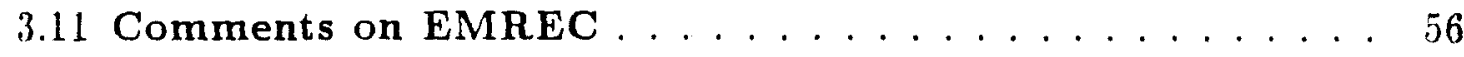

3.12 Track reconstruction and vertex determination $\ldots \ldots \ldots 56$

4 Data analysis $\quad 58$

4.1 General considerations . . . . . . . . . . . 58

4.2 Vertex definition $\ldots \ldots \ldots \ldots \ldots \ldots \ldots$

4.3 Pedestal and gain corrections . . . . . . . . . . 59

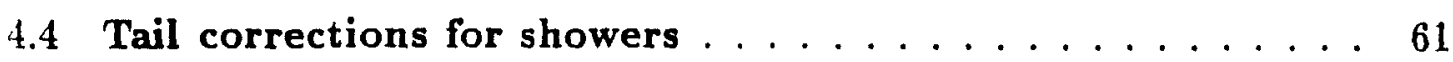

4.5 Definition of the $\pi^{0} \operatorname{signal} \ldots \ldots \ldots 63$ 
46 Background subtraction $\ldots \ldots \ldots \ldots \ldots \ldots$

4.7 Determination of the incident beam . . . . . . . 71

5 Monte Carlo corrections $\quad 73$

5.1 General organization $\ldots \ldots \ldots \ldots \ldots \ldots$

$5.2 \pi^{0}$ generation and decay $\ldots \ldots \ldots \ldots \ldots \ldots$

5.3 Calculation of the Monte Carlo corrections . . . . . . . . it

5.4 Comparison of the Monte Carlo model with the data . . . 70

6 Results and conclusions $\quad 80$

6.1 General considerations . . . . . . . . . . . . . . 80

6.2 Cross section normalization . . . . . . . . . . . 80

6.3 Inclusive cross sections for $\pi^{0}$ production . . . . . . . . 81

6.4 Atomic dependence of inclusive hadronic cross sections . . 87

6.5 Sources of systematic error . . . . . . . . . . . . 87

6.6 Conclusions . . . . . . . . . . . . . . . . 92

Bibliography . . . . . . . . . . . . . . . 99 


\section{List of Tables}

2.1 Target parameters. . . . . . . . . . . . . . . . 19

3.1 Shower shape parameters . . . . . . . . . . . . . 34

3.2 Parameters for the determination of the shower position . . . . . 39

4.1 Number of triggers . . . . . . . . . . . . . . . 59

4.2 Dependence of the $\pi^{0}$ mass on the EMLAC quadrant . . . . . . 6 66

4.3 Fitted values for the $\pi^{0}$ mass and width $\ldots \ldots \ldots 66$

4.4 Cuts for Al data $\ldots \ldots \ldots \ldots \ldots \ldots$

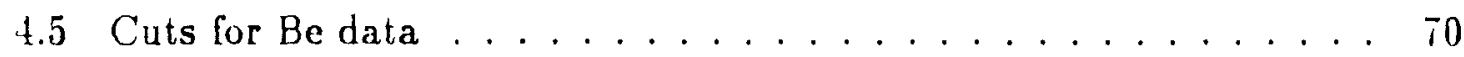

4.6 Interaction counters for Run 2375 (Al target) $\ldots \ldots \ldots 72$

5.1 Number of peaks per "group" for Run $2379(\mathrm{Be}) \ldots \ldots \ldots \ldots$ it

$6.1 \frac{1}{\pi \Delta y} \frac{d \sigma}{d p_{T}^{2}}\left(m b /(G e V / c)^{2}\right)$ (per nucleus) for $\pi^{0}$ production in collisions at $530 \mathrm{GeV},-0.25<y_{c m}<0.25 \ldots \ldots \ldots \ldots 2$

$6.2 \frac{1}{\pi \Delta y} \frac{d \sigma}{d p_{T}^{2}}\left(m b /(G e V / c)^{2}\right)$ (per nucleus) for $\pi^{0}$ production in collisions at $530 \mathrm{GeV}, 0.25<y_{\mathrm{cm}}<0.75 \ldots \ldots \ldots \ldots 2$

6.3 The value of the power $\alpha$ in $A^{\alpha}$ for $\pi^{0}$ production in collisions at 530 $\mathrm{GeV},-0.25<y_{\mathrm{cm}}<0.25 \ldots \ldots \ldots \ldots \ldots$ 
6.4 The value of the power $\alpha$ in $A^{\alpha}$ for $\pi^{0}$ production in collisions at 530 $\mathrm{GeV}, 0.25<y_{c m}<0.75 \ldots \ldots \ldots \ldots \ldots$

6.5 The value of the power $\alpha$ in $A^{\alpha}$ for $\pi^{0}$ production in collisions at 530 $\mathrm{GeV}$ as a function of $p_{T},-0.25<y_{\mathrm{cm}}<0.75 \ldots \ldots \ldots$

6.6 The value of the power $\alpha$ in $A^{\alpha}$ for $\pi^{0}$ production in collisions at 530 $\mathrm{GeV}$ as a function of center of mass rapidity, $0.7 \mathrm{GeV} / \mathrm{c}<p_{T}<2.1$ $\mathrm{GeV} / \mathrm{c} \ldots \ldots \ldots \ldots \ldots \ldots \ldots$

6.7 Integrated inclusive cross sections (mbarns) per nucleus for $\pi^{0}$ production in $\pi^{-} B e$ and $\pi^{-} A l$ interactions at $530 \mathrm{GeV} \ldots \ldots .4$

6.8 Values of the shape parameters in the fits to the inclusive cross sections for $\pi^{0}$ production in collisions at $530 \mathrm{GeV},-0.25<y_{\mathrm{cm}}<0.75$ 


\section{List of Figures}

$1.1 d \sigma / d p_{T}^{2}$ distribution for $\pi^{0}$ production from $\mathrm{NA} 22 \ldots \ldots \ldots \ldots$

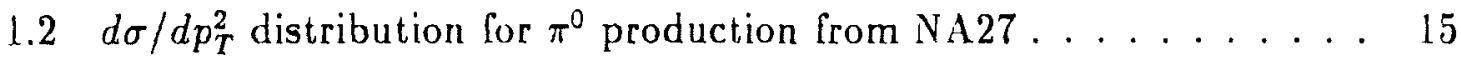

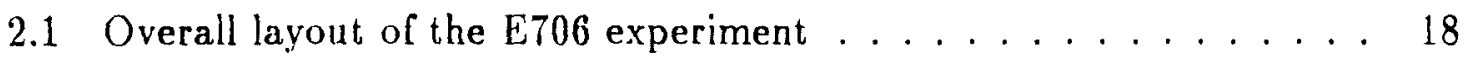

2.2 An exploded view of the EMLAC stack . . . . . . . . . 23

$2.3 \quad$ E706 on-line system and LAC readout electronics . . . . . . . 25

2.4 E706 LAC amplifier module . . . . . . . . . . . 27

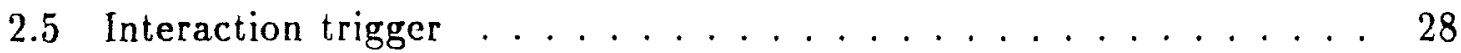

3.1 Some representative "groups" of showers . . . . . . . . . . 37

3.2 Energy correlation between $\mathrm{r}$ and $\phi$ view $\ldots \ldots \ldots \ldots \ldots \ldots$

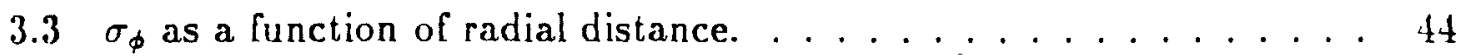

3.4 Resolution in $\mathrm{r}$ prediction from shower width in $\phi . \ldots \ldots \ldots$

3.5 Distribution of the square of the width of showers . . . . . . . . 46

3.6 Walker mass distribution . . . . . . . . . . . . . . 47

3.7 Position resolution for Monte Carlo photons . . . . . . . . . . 49

3.8 Energy resolution for Monte Carlo photons . . . . . . . . . 50

3.9 Two-photon mass distribution for single $\pi^{0}$ events $\ldots \ldots \ldots 1$ 
3.10 Reconstructed asymmetry. . . . . . . . . . . . . . . 52

3.11 Two-photon mass distribution for multi- $\pi^{0}$ events. . . . . . . . 54

3.12 Two-photon mass distribution for multi- $\pi^{0}$ events, for $p_{T}>5 \mathrm{GeV} / \mathrm{c} \quad 55$

$4.1 \mathrm{z}$ distribution of primary interaction vertices. . . . . . . 60

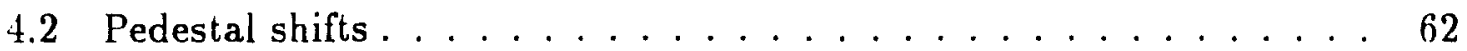

4.3 Energy below threshold . . . . . . . . . . . . . . 64

$4.4 \gamma \gamma$ invariant mass distribution for Be data. . . . . . . . 68

$4.5 \gamma \gamma$ invariant mass distribution for Al data. . . . . . . . 69

5.1 Monte Carlo weights versus $p_{T}$ and rapidity $\ldots \ldots \ldots \ldots$

5.2 Asymmetry distribution for $\pi^{0}$ production from the Monte Carlo prediction for negative data . . . . . . . . . . . 77

$5.3 \gamma \gamma$ mass distribution for Monte Carlo $\pi^{0}$ 's at low $p_{T} \ldots \ldots \ldots 79$

$6.1 \frac{1}{\pi \Delta y} \frac{d \sigma}{d p_{T}^{2}}$ for $\pi^{0}$ production in $\pi^{-} B e$ collisions at $530 \mathrm{GeV},-0.25<$ $y_{c m}<0.25 \ldots \ldots \ldots \ldots \ldots \ldots \ldots$

$6.2 \frac{1}{\pi \Delta y} \frac{d \sigma}{d p_{T}^{2}}$ for $\pi^{0}$ production in $\pi^{-} B e$ collisions at $530 \mathrm{GeV}, 0.25<y_{c m}<$ $0.75 \ldots \ldots \ldots \ldots \ldots \ldots \ldots \ldots \ldots$

$6.3 \frac{1}{\pi \Delta y} \frac{d \sigma}{d p_{T}^{2}}$ for $\pi^{0}$ production $\pi^{-} A l$ collisions at $530 \mathrm{GeV},-0.25<y_{c m}<$ $0.25 \ldots \ldots \ldots \ldots \ldots \ldots \ldots \ldots \ldots \ldots$

$6.4 \frac{1}{\pi \Delta y} \frac{d \sigma}{d p_{T}^{2}}$ for $\pi^{0}$ production in $\pi^{-} A l$ collisions at $530 \mathrm{GeV}, 0.25<y_{\mathrm{cm}}<$ $0.75 \ldots \ldots \ldots \ldots \ldots \ldots \ldots \ldots \ldots \ldots$

6.5 The value of the power $\alpha$ in $A^{\alpha}$ for $\pi^{0}$ production, $-0.25<y_{\mathrm{cm}}<0.2588$

6.6 The value of the power $\alpha$ in $A^{\alpha}$ for $\pi^{0}$ production, $0.25<y_{\mathrm{cm}}<0.75 \quad 89$

6.7 Fit to $\frac{1}{\pi \Delta y} \frac{d \sigma}{d p_{T}^{2}}$ for $\pi^{0}$ production in $\pi^{-} A l$ collisions, $-0.25<y_{c m}<0.7596$ 
6.8 Fit to $\frac{1}{\pi \Delta y} \frac{d \sigma}{d p_{T}^{2}}$ for $\pi^{0}$ production in $\pi^{-} .11$ collisions, $-0.25<y_{c m}<0.7597$ 


\section{Chapter 1}

\section{INTRODUCTION}

\subsection{General considerations}

The results presented in this thesis come from Fermilab experiment E706. The main goal of this experiment is the measurement of direct photon yields in the transierse momentum $\left(p_{T}\right)$ range of $4-10 \mathrm{GeV} / \mathrm{c}$, with incident $\pi / K / p$ beams at $500-600 \mathrm{GeV}$, as well as the study of associated jets produced in such reactions. This thesis will present a measurement of the inclusive cross section for $\pi^{0}$ production at lower $p_{T}$ $(0.6-2.2 \mathrm{GeV} / \mathrm{c})$ in $\pi^{-} B e$ and $\pi^{-} A l$ interactions.

Data on neutral mesons decaying into photons, at center of mass energies larger than $\sqrt{s}=15 \mathrm{GeV}$ are scarce compared to data on inclusive charged mesons and neutral strange particle production; any new measurement is therefore of value for testing models of parlicle production and of use in gauging backgrounds for other experiments at high energy.

This thesis is divided into six chapters. We first continue with general issues of phenomenology and recent data. Chapters $2-4$ concern the experimental apparatus and analysis techniques. Chapter 5 deals with a simulation of the detector, and Chapter 6 provides a discussion of the results and conclusions. 


\subsection{Theoretical models}

Since the time of Yukawa [1] little progress has been made in the understanding at a fundamental level of low momentum transfer hadronic interactions; even if quantum chromodynamics (QCD) [2] emerged as a candidate theory of strong interactions, a perturbative solution for $Q C D$ can be found only at high $Q^{2}\left(Q^{2}\right.$ is the square of an appropriate momentum transfer which characterizes the collision).

It is hoped that an understanding of low $Q^{2}$ phenomena will eventually come from new mathematical techniques which will encompass the non-perturbative region in QCD (or in other theories). In the meanwhile, even if high $Q^{2}$ processes get the most attention, it is also important to gather experimental data in the soft region.

The present theoretical description of low $p_{T}$ physics remains at the level of ad hoc phenomenology $[3,4,5,6]$, which will not be described in detail here. These models are naturally conceived with the intent of explaining existing data. They typically replace the strong interaction problem with assumptions regarding the precollisional structure of hadrons, the rearrangement of the partons into a final state and the subsequent transformation of this system into hadrons.

\subsection{Kinematics variables}

The quantity of fundamental importance in the description of the scattering of two particles is the cross section. 'The cross section for a process measures the probability for the occurrence of a particular rcaction. To gain an understanding of the dynamics of strong interactions, one could study as many exclusive channels as are accessible to experimental investigation; this approach however is not manageable in general because many exclusive channels are too complicated to be reconstructed experimentally and the particle multiplicitics are large.

In the following we will deal with inclusive single-particle reactions of the kind [7]:

$$
A+B \rightarrow C+A N Y T I I N G
$$


Reaction (1.1), which gives the production of a specific particle C, accompanied by any other particles produced in a collision of particles $A$ and $B$, can be described by a Lorentz-invariant function characterizing the production process: this is a differential invariant single-particle production cross section, a quantity proportional to the probability for emitting a specific particle $\mathrm{C}$ into a Lorentzinvariant element of momentum space:

$$
\frac{E_{c} d^{3} \sigma\left(p_{c}, s_{a . b}\right)}{d p_{c}^{3}}
$$

In equation (1.2) we have explicitly indicated all the possible kinematic dependence that the quantities of physical interest might have. The total cross section for reaction (1.1) can depend on the energy of the scattering system: $s_{a, b}$, or simply $s$, is the square of the total energy available in the center of mass frame of the scattering:

$$
s_{a, b}=\left(p_{a}+p_{b}\right)^{2}=\left(E_{a}+E_{b}\right)^{2}-\left(\overrightarrow{p_{a}}+\overrightarrow{p_{b}}\right)^{2}
$$

The variables $\vec{p}_{c}, E_{c}, p_{c}$ are the vector momentum, total energy and four-momentum of particle $\mathrm{C}$ and $d^{3} p_{c}$ is the differential element of 3 -momentum space into which particle $\mathrm{C}$ is emitted.

Since we used only unpolarized incident projectile and target particles, we do not expect to observe any azimuthal dependence in the production of particle $\mathrm{C}$. We assume therefore that the dependence on $p_{c}$ will reduce to a dependence on the longitudinal momentum $\left(p_{1}\right)$ and the transverse momentum $\left(p_{T}\right)$ of particle $\mathrm{C}$.

The $p_{T}$ variable is invariant under a Lorentz transformation along the line of collision between the incident particles. Instead of using the longitudinal and transverse momentum, one can use the normalized variables:

$$
x_{F}=\frac{p_{l}}{p_{l, \max }}
$$

where $p_{l}$ is the longitudinal mornentum in the center of mass frame, and $p_{l, \max }$ is its maximum value $\left(p_{l, \max }=\frac{\sqrt{3}}{2}\right)$; and

$$
x_{T}=\frac{p_{T}}{p_{T, \max }}
$$

where $p_{T}$ is the tranverse momentum in the center of mass frame, and $p_{T, m a x}$ is its maximum value $\left(p_{T, \max }=\frac{\sqrt{3}}{2}\right)$. 
Another useful variable is the rapidity:

$$
y=\frac{1}{2} \ln \frac{E+p_{l}}{E-p_{l}}
$$

The rapidity is additive under Lorentz transformations along the collision axis. The pseudorapidity variable is more convenient than the rapidity from the experimental point of view. It is defined as :

$$
\eta=-\ln \left(\tan \frac{\theta}{2}\right)
$$

( $\theta$ is the angle at which particle $\mathrm{C}$ is emitted with respect to the line of collision between the incident particles). The pseudorapidity approximates the rapidity for ultrarelativistic particles.

\subsection{Scaling and limiting fragmentation}

In this section the concepts of scaling and limiting fragmentation $[8,9]$ will be introduced.

Experimental evidence suggests that total cross sections for various incident channels exhibit rather small changes at high energies. One can study whether all inclusive invariant cross sections approach energy independent forms (hypothesis of limiting fragmentation). This implies that at fixed $p_{T}$ the cross section for Reaction (1.1) at any value of $p_{l}$ (as measured in either the rest frame of $\mathrm{A}$ or B) will approach a finite energy-independent limit; because at fixed $p_{T}$ the energy of particle $\mathrm{C}$ as measured in either the rest frame of particle $A$ or $B$ is determined by the mass and the $p_{l}$ value of particle $C$, the limit should be valid for the invariant cross section for Reaction (1.1) as well as for the more standard non Lorentz-invariant cross section; that is

$$
E d \sigma / d p^{3}\left(p_{l}, p_{T}, s_{a b}\right) \rightarrow E d \sigma / d p^{3}\left(p_{l}, p_{T}\right)
$$

or

$$
d \sigma / d p^{3}\left(p_{l}, p_{T}, s_{a b}\right) \rightarrow d \sigma / d p^{3}\left(p_{l}, p_{T}\right)
$$

(the above limits are valid for $s_{a b} \rightarrow \infty$ for any finite $p_{T}$ and $p_{l}$ of particle $\mathrm{C}$ as measured in the rest frames of the target or of the projectile particle). 
Feynman suggested that hadronic particle emission at very high energies should be similar to the radiation of photons by an accelerating charge; for fixed $p_{T}$, the probability for the emission of particle $\mathrm{C}$ in the range of momentum $d p_{l}$ in a collision between particles $A$ and $B$ should have the energy dependence typical of Bremsstrahlung processes:

$$
d \sigma \propto(1 / E) d p_{1}
$$

If we assume also that the fraction of incident longitudinal momentum $x$ carried away by any produced particles should become $s$-independent, we obtain the Feynman scaling hypothesis (for $s_{a b} \rightarrow \infty$ for all $p_{T}$ and $p_{l}$ values):

$$
E d \sigma / d p^{3}\left(p_{l}, p_{T}, s_{a b}\right) \rightarrow E d \sigma / d p^{3}\left(x, p_{T}\right)
$$

where the above quantities are given in the CM frame.

\subsection{Experimental measurements of low $p_{T}$ neutral pion production}

The most recent determinations of $\pi^{0}$ yields came from the NA22 Collaboration at CERN [10], which measured inclusive $\pi^{0}$ production in $\pi^{+} p, K^{+} p$ and pp interactions at $250 \mathrm{GeV}$ and from the NA27 Collaboration at CERN [11], which measured the inclusive $\pi^{0}$ and $\eta^{0}$ production in $\pi^{-} p$ at $360 \mathrm{GeV}$. The $d \sigma / d p_{T}^{2}$ distribution for $\pi^{0}$ 's from NA22 for $x_{F}\left(\pi^{0}\right)>0.025$ is given in Fig. [1.1]; the distribution is well fitted with a sum of two exponentials.

The $d \sigma / d p_{t}^{2}$ distribution for $\pi^{0}$ s from NA27 is given in Fig. 1.2. Since the $p_{t}$ range of NA27 extends to higher values than the range for experiment NA22, they chose to fit the distribution in Fig. 1.2 with a function of the form:

$$
d \sigma / d p_{T}^{2}=\frac{C}{\left(p_{T}^{2}+\delta^{2}\right)^{n}}
$$

where $\delta=0.64 \pm 0.02 \mathrm{GeV} / \mathrm{c}$ and $n=4.03 \pm 0.07$.

Earlier studies of $\pi^{0}$ production for $K^{+} p$ interactions [12,13,14] and for $\pi^{+} p$ interactions $[15,16,17,18,19,20,21,22]$ at lower energies either suffer from low statistics or small acceptance. 
Figure 1.1: $d \sigma / d p_{T}^{2}$ distribution for $\pi^{0}$ production at $250 \mathrm{GcV}$ from NA22 for $x_{F}\left(\pi^{0}\right)>0.025$

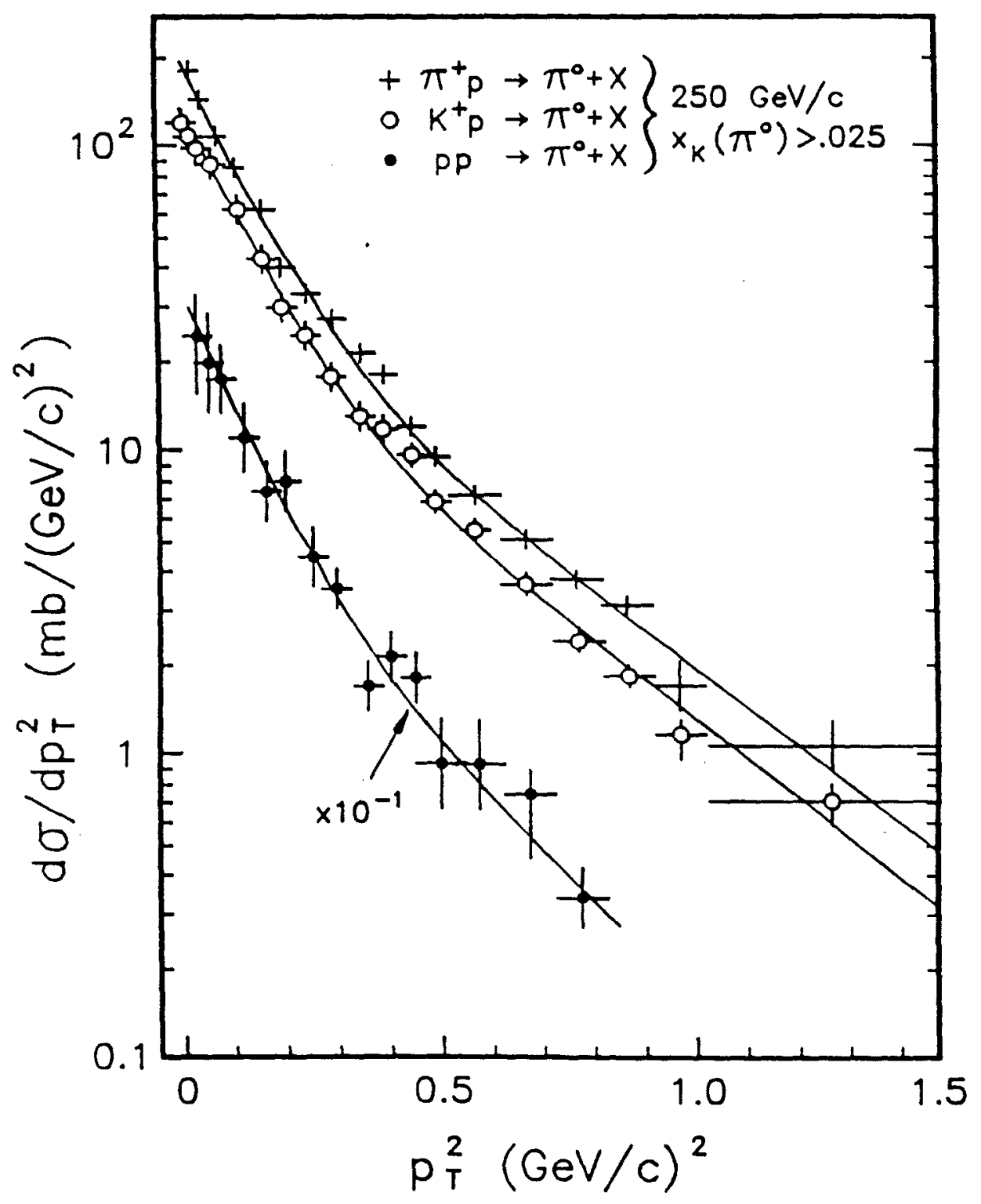


Figure 1.2: $d \sigma / d p_{T}^{2}$ distribution for $\pi^{n}$ production from NA27 for $x_{F}\left(\pi^{0}\right)>0.01$ at $360 \mathrm{GeV}$

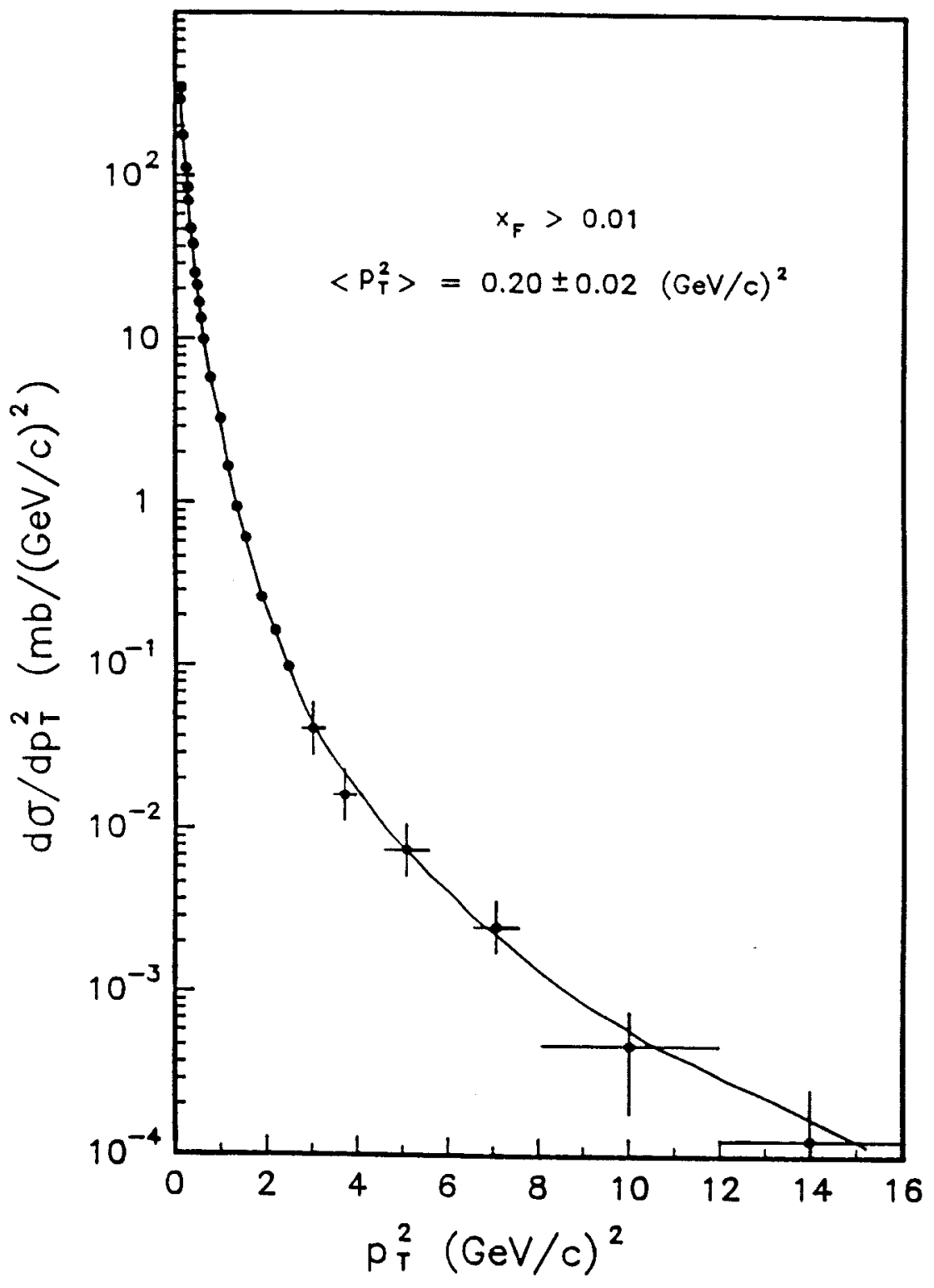




\subsection{Atomic dependence of inclusive hadronic cross sections}

High energy hadron-nucleus scattering reactions probe the space-time development of the hadronic production process. Invariant inclusive hadronic cross sections from reactions in different nuclei (different atomic number A) are conventionally parametrized to display the $A$ dependence as a single power $\alpha$ of $A$ [23]:

$$
E d \sigma / d p^{3}\left(A, x_{F}, \overrightarrow{p_{T}}\right)=A^{\alpha\left(x_{F}, \vec{T}\right)} E d \sigma / d p^{3}\left(1, x_{F}, \overrightarrow{p_{T}}\right)
$$

The power $\alpha$ depends in general on $x_{F}$ and $\overrightarrow{p_{T}}$, where the dependence on $x_{F}$ and $\overrightarrow{p_{T}}$ may also vary with $A$. Some data have been analyzed using a series in powers of $A^{1 / 3}$ to fit the cross section:

$$
d \sigma / d y=A^{2 / 3}\left[a(y)+A^{1 / 3} b(y)+A^{2 / 3} c(y)\right]
$$

In the previos equation the cross section is either at fixed $\overrightarrow{p_{T}}$ or has been integrated over $\overrightarrow{p_{T}}$ and is expressed in terms of the rapidity y. The total absorption cross section exhibits an $A^{2 / 3}$ dependence, as can be expected from geometric shadowing considerations [24]. Naively one may imagine that in hard collisions nucleons would behave as free particles. It has been observed however $[25,26,27]$ that the power $\alpha$ is a function of $p_{T}$ and for all particle types grows to be larger than one at large $p_{T}$, implying that nucleons must behave in a cooperative manner (i.e. a rescattering of the outgoing state may occur). A number of models have been proposed to explain the features of hadron-nucleus collisions at high energy $[28,29,30,31,32]$ but the theoretical understanding is still limited. 


\section{Chapter 2}

EXPERIMENTAL APPARATUS

\subsection{General considerations}

This chapter describes the apparatus used in the experiment, with particular emphasis on the electromagnetic calorimeter; the data acquisition procedure is also mentioned.

Figure [2.1] shows the overall layout of the experiment, which was designed to cover as much of the total solid angle as possible.

A right-handed coordinate system with the positive $z$-axis directed downstream along the beam line, the positive $y$-axis vertically upward and with the origin located $12 \mathrm{~cm}$ downstream of the midpoint of the target segments will be used in the following discussion.

\subsection{Beamline}

The experiment used the Meson West (MW) beamline at Fermilab [33]. The beam is a high resolution and high intensity secondary beam with mass definition provided by a Cherenkov counter. The MW beam line is made up of two stages: it has a first focus, a parallel section to accommodate the Cherenkov counter, and a final focus. 
Figure 2.1: Overall layout of the E706 experiment

a)

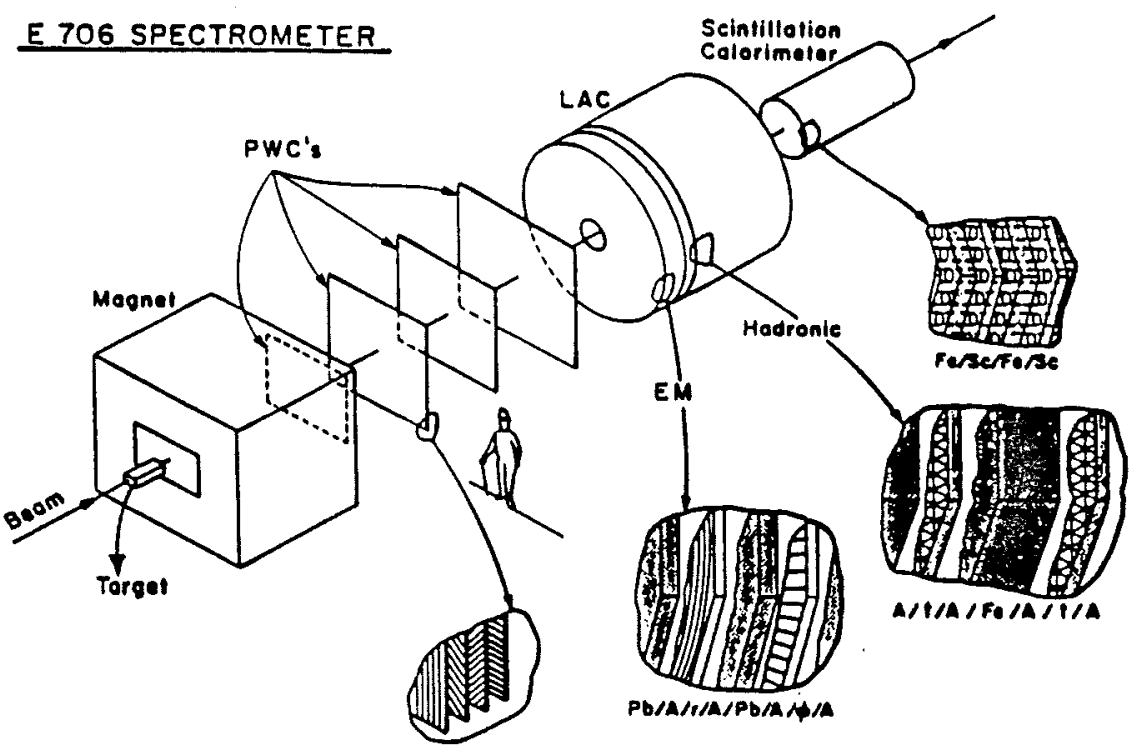

b) TARGET AND SILICON MICROSTRIP SYSTEM

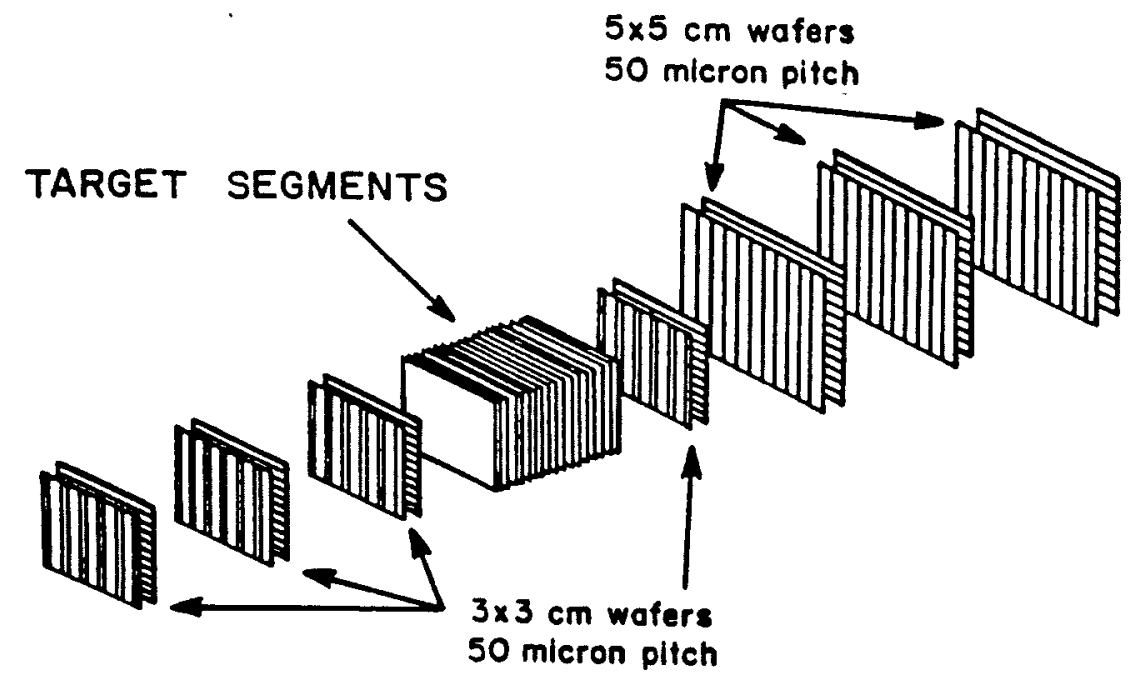


Table 2.1: Target parameters.

\begin{tabular}{|l|c|c|c|}
\hline Parameters & $\mathrm{Be}$ & $\mathrm{Ml}$ & $\mathrm{Si}$ \\
\hline length $(\mathrm{cm})$ & 4.00 & 0.95 & 0.42 \\
\hline atomic number & 9.01 & 26.98 & 28.09 \\
\hline density $\left(\mathrm{g} / \mathrm{cm}^{3}\right)$ & 1.85 & 2.70 & 2.33 \\
\hline radiation length $(\%)$ & 11.3 & 11.7 & 4.4 \\
\hline$\pi$ interaction length $(\%)$ & 6.8 & 1.8 & 0.7 \\
\hline p interaction length $(\%)$ & 9.2 & 2.3 & 0.9 \\
\hline
\end{tabular}

During most of the E706 data taking, we ran with a momentum bite of about $\pm 5 \%$ and a typical intensity of $50 \times 10^{6}$ particles over a 20 sec spill time.

\section{$2.3 \quad$ Targets}

We used nuclear targets because they are compact and easy to handle and they are interesting in their own right. The choice of target material was based on considerations of radiation length and interaction length. If a photon from a $\pi^{0}$ converts to an $e^{+} e^{-}$pair within a target, the charged particles can undergo multiple Coulomb scattering, and mimic the production of two photons, if the opening angle is appreciable. To avoid converting too many $\pi^{0}$ 's, the scaling properties of the radiation length and the interaction length as functions of atomic weight and atomic number suggest the use of relatively low $\mathrm{Z}$ material for targets.

The target was split into several segments. We used a $10 \%$ absorption length carbon target for most of our standard direct photon data taking; however, all of the interaction triggers which comprise the data for this thesis, were taken with beryllium and aluminum targets, chosen to have the same radiation lengths. The characteristics of the targets and of the silicon material constituting the main silicon detectors (SSD's) are given in Table [2.1]. 


\subsection{Charged particle detection}

A large-aperture magnet, with a maximum field integral of about $1 \mathrm{Tm}$, provided excellent acceptance for charged particles.

In the region in front of the magnet we have an array of silicon strip detectors (SSD) $[34,35,36]$, while downstream of the magnet we have a tracking system consisting of 16 planes of multiwire proportional chambers (MWPC) [37].

The beam enters the segmented production target through three pairs of crossed (X-I) SSD planes. Immediately downstream of the target are four additional sets of $\mathrm{X}-\mathrm{Y}$ modules for monitoring the charged hadrons produced in a collision. Hence, there are a total of 14 SSD planes which have approximately 6000 readout channels, and a pitch (width of each strip) of $50 \mu$. At this time, the SSD outputs are not instrumented for providing pulse height information.

The SSD system has to establish which hadrons emerging from the target are due to secondary interactions in the target. The size of the system is large enough so that its readout does not shadow the downstream liquid argon calorimeter, nor the acceptance aperture that is set by the magnet opening. The first four doublets are $3 \mathrm{~cm} \mathrm{X} 3 \mathrm{~cm}$ and the last three are $5 \mathrm{~cm} X 5 \mathrm{~cm}$, in transverse dimensions, and the silicon wafers are all approximately $300 \mu$ thick.

The MWPC's have high rate capability ( $>1 \mathrm{MHz}$ interaction rate ) and good angular segmentation $(<1 \mathrm{mr})$. We have 16 planes packaged into four modules with an $x, y, u\left(+37^{\circ}\right), v\left(-53^{\circ}\right)$ orientation of the sense wires in each module. The wires are $20 \mu$ gold plated tungsten. Mylar sheets, spray painted with graphite, form the cathodes of the chambers. The four modules have $1.62 \mathrm{~m} \mathrm{X} 1.21 \mathrm{~m}, 2.03$ $\mathrm{m} \mathrm{X} 2.03 \mathrm{~m}, 2.03 \mathrm{~m} \mathrm{X} 2.03 \mathrm{~m}, 2.44 \mathrm{~m} \mathrm{X} \mathrm{2.44} \mathrm{m} \mathrm{transverse} \mathrm{dimensions,} \mathrm{and} \mathrm{wire}$ spacings of $2.54 \mathrm{~mm}$.

\subsection{Liquid argon calorimeter}

The specifications and characteristics of the calorimeter can be summarized as follows: 
1. Good acceptance for both photons and hadrons.

2. Energy resolution of $<15 \% \sqrt{\text { Energy }}(\mathrm{GeV})$ and a position resolution $<0.1$ $\mathrm{cm}$ for photons or electrons. The two-particle resolution is particularly important for distinguishing single photons from photon pairs due to decays of high energy $\pi^{0}$ s and is about $1 \mathrm{~cm}$.

3. Hadron energy resolution of $100 \% \sqrt{\text { Energy }}(\mathrm{GeV})$, with a position resolution of a few centimeters.

4. Very good discrimination between photon showers and those initiated by neutral hadrons.

5. Ability to sustain an interaction rate of $1 \mathrm{MHz}$.

The liquid argon calorimeter ( $\mathrm{LAC})[38,39]$ is located $9 \mathrm{~m}$ from the target region. It measures the position and energy of photons, electrons and hadrons. It consists of an electromagnetic calorimeter section (EMLAC) and a hadron calorimeter (HALAC), in a common cryogenic enclosure.

The photon detector is subdivided into four mechanically independent quadrants, held together by the overall support structure. Each quadrant consists of 66 layers in which $2 \mathrm{~mm}$ lead plates are separated by $2.5 \mathrm{~mm}$ gaps of liquid argon from copper-clad G-10 readout boards. Each readout board comprises an octant of the detector. Alternate boards are used to read out the $r$ cooordinate and the $\phi$ coordinate in a polar coordinate system. The $\mathrm{r}$ coordinate readout consists of 256 concentric radial strips in each octant; the strips are focussed in tower fashion towards the target. The $\phi$ coordinate strips are subdivided into inner and outer segments; in each octant there are 48 inner $\phi$ strips and 96 outer $\phi$ strips.

Each lead plate is connected to a negative high voltage supply through $200 \mathrm{nF}$ of capacitance (two $100 \mathrm{nF}$ capacitors used in parallel and a $10 \Omega$ series resistor). This reduces voltage sagging during the beam spill.

The support structure of each quadrant, as well as the additional space needed for readout connectors and G-10 spacers (to separate the boards from each other and from the lead) produce dead areas both for incident photons and hadrons. Reconstruction is essentially impossible for photons that hit the support structure or the G-10 spacers, and for all hadrons that hit the stainless steel support plates; however, hadrons that hit the G-10 spacers can be detected. 
The electromagnetic calorimeter is segmented into two sections; the first $1 / 3$ of the detector ( 22 layers, 10 radiation lengths) is read out via the front readout boards, the last $2 / 3$ ( 44 layers, 20 radiation lengths) is read out via the back readout planes. Figure [2.2] displays some of the above mentioned details.

In contrast to the photon detector, the hadron detector [40] has a tower readout structure. Each tower consists of a series of triangular pads. The hadron detector is not described in detail here, since it was not used in this analysis.

Both the photon and the hadron calorimeters are suspended from the cover plate of a $17 \mathrm{ft}$ diameter upright cylindrical cryostat. The cover plate is attached to a moveable gantry that moves on rails perpendicular to the beam direction; the beam can be swept vertically by a pitching magnet located at $848.6 \mathrm{ft}$. from the primary target; we can therefore sweep the beam across almost any portion of the calorimeter for calibration and testing.

\subsection{Operation of the calorimeter}

The calorimeter is operated as an ionization chamber; there is therefore no electron multiplication of the signal.

Electromagnetic showers are generated in the the lead plates; charged particles in the shower deposit energy in the argon (active medium) by ionizing the atoms; the shower energy is then measured by collecting the ionization charge. For our geometry, about $20 \%$ of the shower energy is deposited in the liquid argon.

The main advantages of using liquid argon as the active medium are:

- It provides a uniform response over the active area.

- It allows a stable operation over long periods of time.

- It is not electronegative and it has a high electron mobility $(\approx 5 \mathrm{~mm} / \mu \mathrm{s}$ at $1 \mathrm{kV} / \mathrm{mm}$ ); in addition, many electronegative impurities are frozen out in liquid argon. This means that the ionization electrons are not absorbed in the medium.

- It is dense $\left(\approx 1.4 \mathrm{gr} / \mathrm{cm}^{3}\right)$, which means that it is easy to obtain large signals; 
Figure 2.2: In exploded view of the EMLAC stack. The front rcadout boards are shown in front of the stack

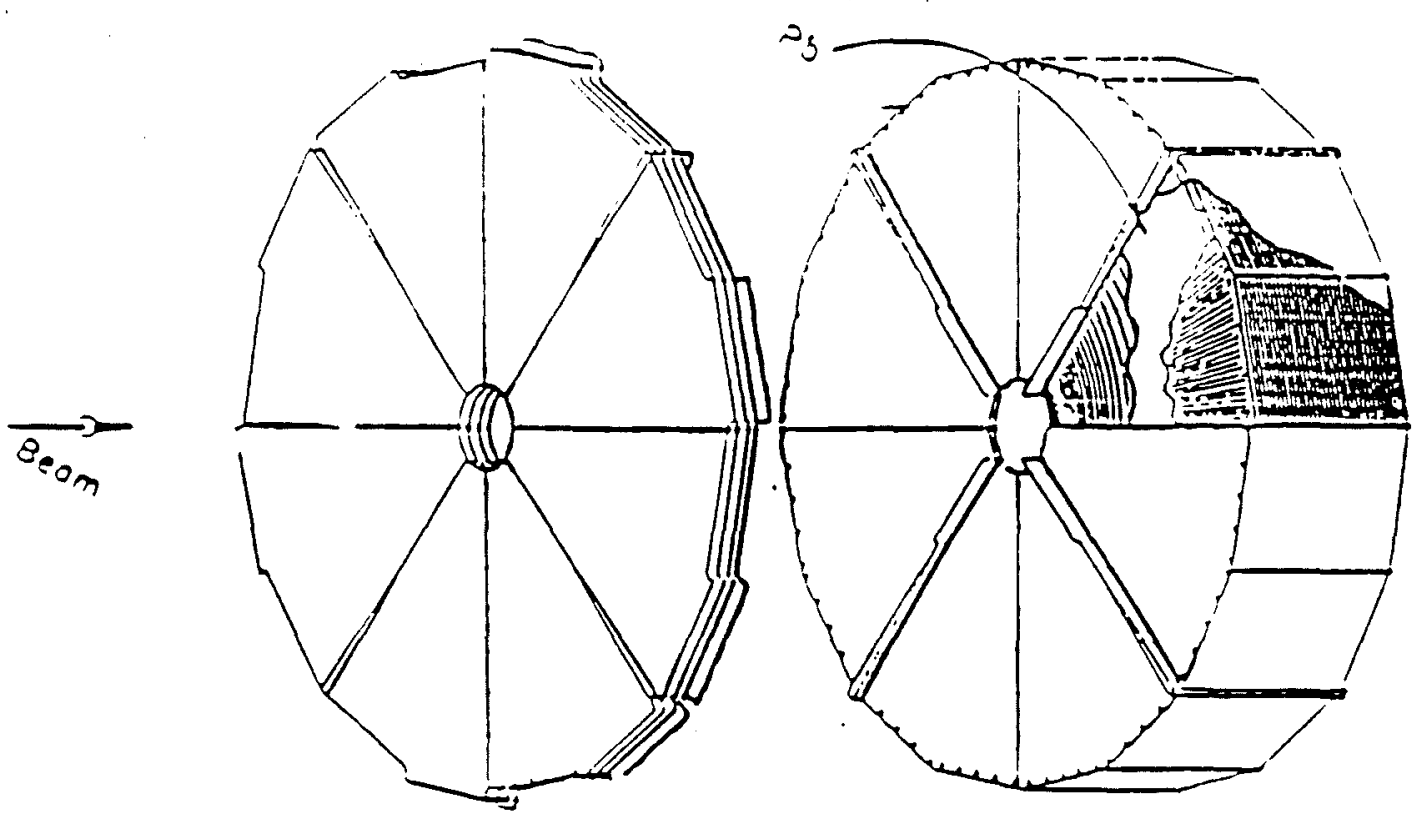


- It is reasonably inexpensive, not difficult to purify and non-flammable.

The main problem of liquid argon is cryogenics; that is, the containment vessel must be maintained at liquid argon temperature $\left(86 \mathrm{~K}^{0}\right)$; nevertheless, the cryostat can be cooled using liquid nitrogenon, which is inexpensive.

The charge collected in the device is due entirely to the electron signal, because the electron drift velocity is 3-4 orders of magnitude greater than that of the positive ions [41]. Since we hold the lead plates at a negative potential, the ionization electrons drift toward the copper-clad boards, which are at ground potential. The collected charge $Q$ can be computed from requirements of energy conservation [42]. The entire signal is not usually observed because some of the drifting electrons recombine with positive ions, and some are trapped by electronegative impurities before reaching the G-10 board.

\subsection{Calorimeter readout electronics}

Figure [2.3] shows the general hard ware scheme for the the LAC readout electronics together with the E706 on-line system, which will be described in Section [2.10], and the EMLAC trigger electronics, which will be introduced in Section [2.8].

The RABBIT system $[43,44]$ developed at Fermilab, is used to read out the signals from the calorimeter. It consists of crate controllers called EWE's, Before After Timers (BAT's) and Front End Electronic Devices (FEED's). It has two buses which allow simultaneous access to two channels within a given crate. Each crate has two EWE's, one for each bus. The EWE performs a threshold test to determine whether or not to digitize a particular signal and subtracts pedestals from detected signals.

The ability to avoid digitizing and reading out a channel if it was below threshold was essential for limiting the amount of information to record per event. The threshold had to be set to a rather large value: the impact of it will be discussed in Chapt. 4. The EWE's are controlled through hard-wired ECL processors (MX's) [45] which can perform a typical instruction (addition) in 125 ns. The MX can also be used to apply linear corrections to the data and can serve as an event buffer. 
Figure 2.3: F,706 on-line system and I,dC, readout electronics

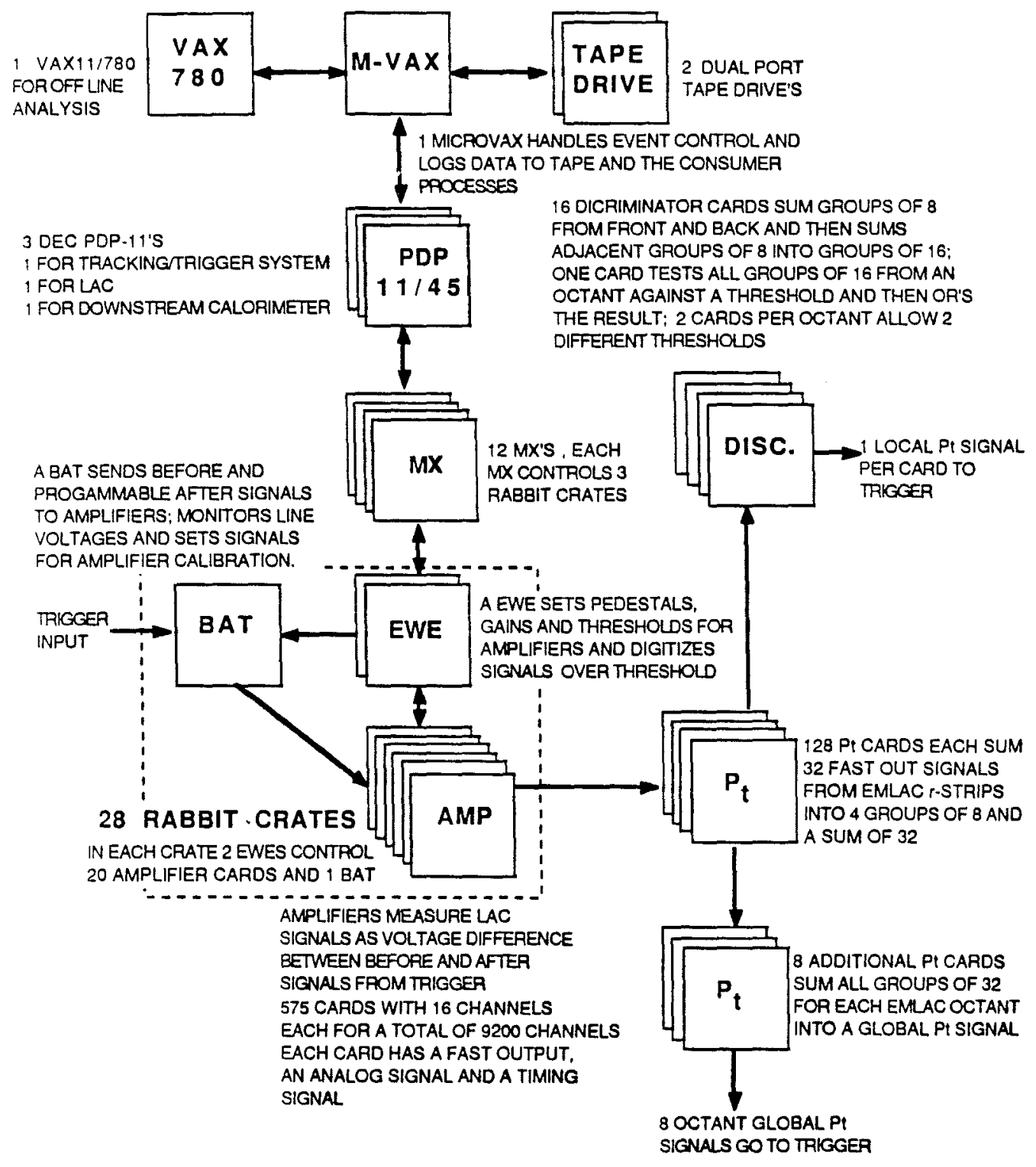


There are two amplifier cards in each rabbit rate. The amplifier is a FEED. Each amplifier card [46] (see Fig. [2.4]) has 16 channels. The output of each channel of the amplifier goes into a $800 \mathrm{~ns}$ delay line to make the signal arrive in time with the trigger information. In addition, a fast output is provided for trigger purposes; it is obtained through delay line differentiation: the output is subtracted from a delayed ( $180 \mathrm{~ns}$ ) copy of itself. The output of each $800 \mathrm{~ns}$ delay line is sampled by two sample-and-hold circuits, each of which is closed by, a different timing signal: the Before and After timing signals generated by the BAT. The Before is timed so that the first sample-and-hold circuit samples the line just before the signal arrives, while the After is timed so that the second sample-and-hold samples the line at the peak of the signal, allowing the EWE to form a difference and thereby subtract the baseline.

Four neighboring channels are also added and fed into a time-to-voltage converter (TVC) sample-and-hold circuit. The TVC start is generated when the sum of four amplifiers exceeds a computer settable threshold; the trigger signal stops the ramp; when the sum of the four amplifiers exceeds this threshold again before the trigger signal arrives, a second TVC records the information for this new pulse. The second TVC is particularly important for high intensity running. The TVC information allows us to remove out of time showers from the reconstructed events.

\subsection{Trigger}

In a dedicated hadronic experiment it is usually necessary to have a trigger to select only a small fraction (typically tens of $\mathrm{Hz}$ ) of the total beam-target interaction rate (which is usually in the MIII range). The data presented in this thesis involve no selectivity and were therefore taken with a low intensity beam (the beam rate varied between 5 and $12 \mathrm{KHz}$ in different runs). We used a special interaction trigger (Fig. [2.5]), with the purpose of collecting unbiased interactions.

A BEAM signal for the trigger is formed by requiring the coincidence of 2 beam counters (BA and $B B$ ) located upstream of the SSD beam chambers, gated by the beam gate BM_GATE (the beam gate is open during the beam spill):

$\mathrm{BEAM}=\mathrm{BM}=\mathrm{BA}$.AND. BB .AND. BM_GATE

An interaction signal INT occurs whenever one of the interaction counters 
Figure 2. I: li706 IdC, amplifier module

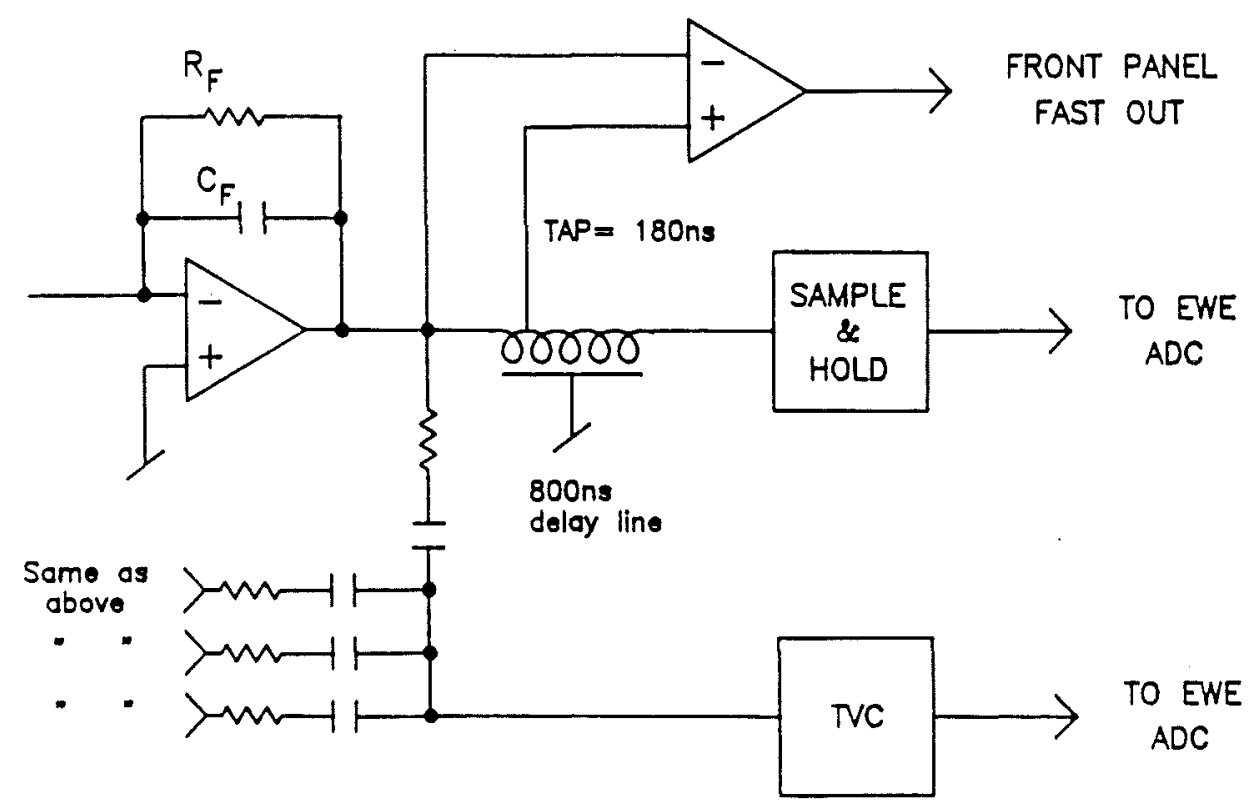


Figure 2.5: Interaction lrigger

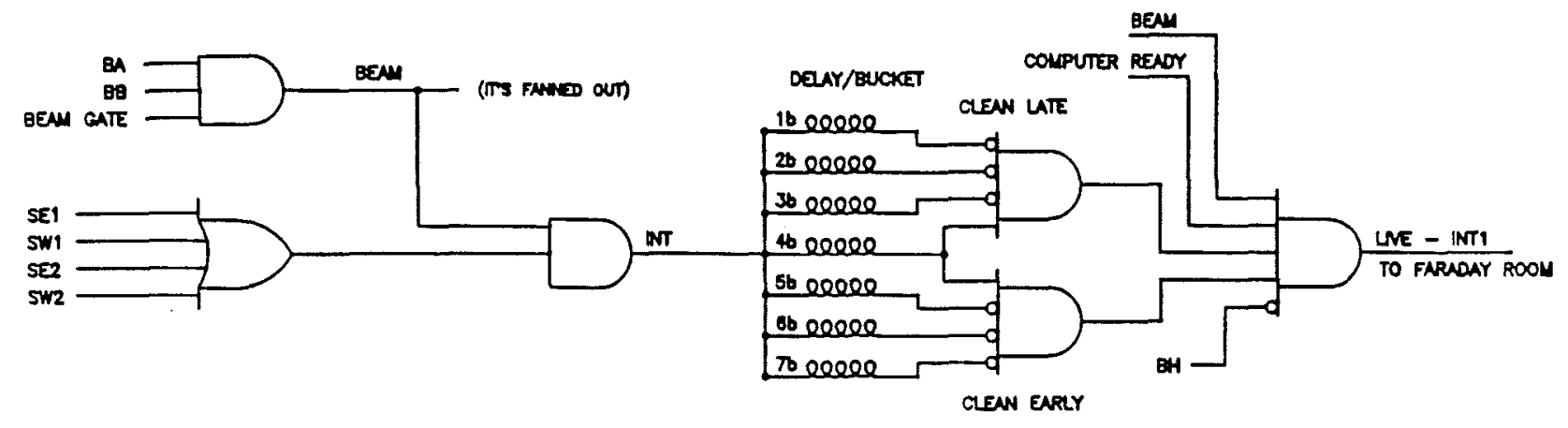

IN FARADAY ROOM

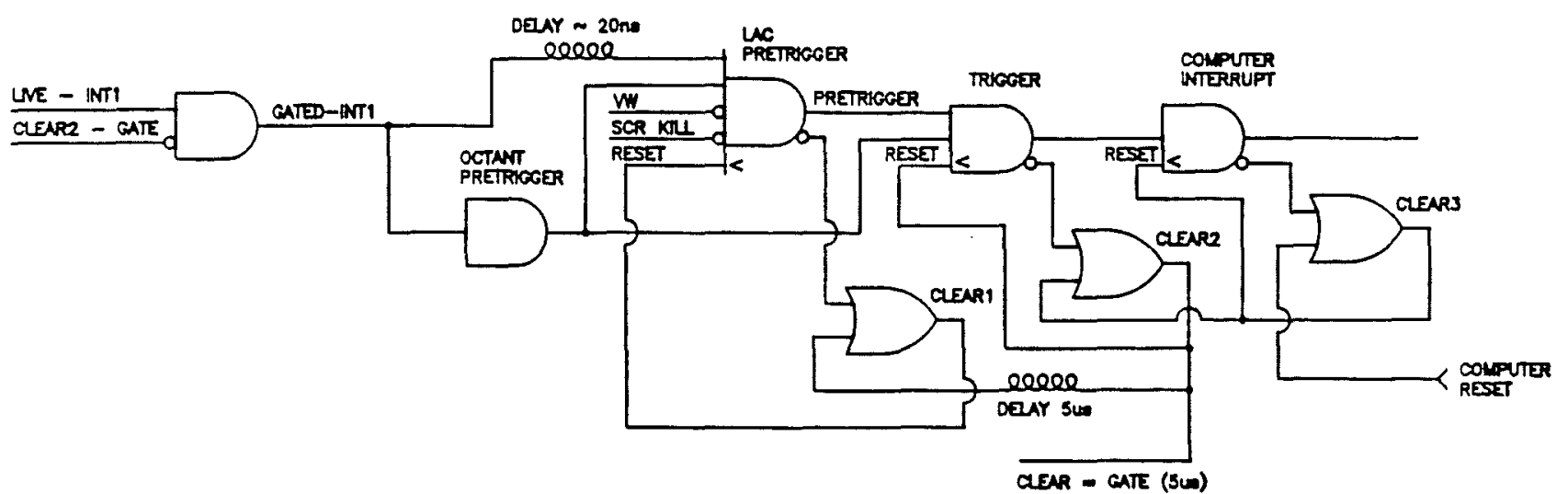


fires in coincidence with the BEAM signal. There are 4 interaction counters: SW1 and SE1, which are located upstream of the magnet, and SW2 and SE2, which are located downstream of it.

INT=BEAM .AND. (SE1.OR.SW1.OR.SE2.OR.SW2)

A clean interaction CLNINT is then defined requiring that no other interaction occurs within \pm 3 buckets of the Fermilab accelerator of (a bucket corresponds to $18.8 \mathrm{~ns})$.

\section{Defining:}

CLEAN-EARLY=INT .AND. (NO INT IN NEXT 3 BUCKETS)

CLEAN_LATE=INT .AND. (NO INT IN PREVIOUS 3 BUCKETS)

we get:

CLNINT $=$ CLEAN_EARLY .AND. CLEAN_LATE

The beam halo counter $\mathrm{BH}$ is used to veto events in which additional particles close to the beam (in the "halo" region) are in time with the beam particle; a signal INT1 is formed from the anticoincidence of BH and CLNINT:

INT $1=$ CLNINT. AND. .NOT. BH

Since it is possible to accept events only when the readout computers are not busy, a signal LIVE-INT1 is defined as the coincindence of INT1 and the computer ready signal.

The standard trigger selects events that appear to have a large amount of $p_{T}$ in a given octant of the electromagnetic calorimeter, with a large fraction of the signal confined to a small spatial region. To obtain the $p_{T}$ in a given region, the pulse height in that channel of the calorimeter is multiplied by the sine of the angle relative to the beam direction. This operation is performed in the main part of the LAC trigger system, using the $p_{T}$ attenuator and discriminator boards, which were designed specifically for the experiment [47]. Each of the $p_{T}$ attenuator boards receives as input 32 'fast out' signals from the signal amplifiers, and produces 4 analog sums of 8 outputs and a single analog sum of 32 outputs. Each of the discriminator boards receives the 'sum-of- 8 ' from the attenuator modules, adds corresponding front and back signals from the LAC, and forms overlapping sums of 
16 adjacent analog signals which go to discriminators. These boards are combined to generate the octant and quadrant $p_{T}$ trigger.

The global $p_{T}$ trigger is susceptible to coherent noise (noise common to all LAC channels, such as pickup from magnet power supplies, radio stations, etc); consequently, we insisted on substantial local deposition of energy in addition to the global $p_{T}$ requirement.

The GATED_INT1 signal, used to strobe the trigger, is formed as a coincidence of LIVE_INT1 and CLEAR2_GATE. The CLEAR2_GATE was closed for $5 \mu$ shenever the BAT had to be reset (that happened when there vas a LAC pretrigger but it fired no trigger, or when there was a computer reset signal).

A LAC PRETRIGGER signal was defined as a coincidence of GATED_INT1 and of a signal from the $p_{T}$ boards, with the anticoincidence of VETO WALL, SCRKILL and EARLY PT. 'To prevent out of time events from contributing to the $p_{T}$ trigger, the EARLY PT was set when there was a signal in the $p_{T}$ board within $300 \mathrm{~ns}$ of the interaction. The VETO WALL was set when both veto walls were hit, indicating that a muon or some particle registered in the counters on the veto wall within $\pm 70 \mathrm{~ns}$ of the interaction. The SCRKILL vetoed any pretrigger if a sizeable and narrow noise spike occurred within $\pm 15 \mu$ s.

The special interaction trigger was implemented by modifying the logic tables of the the standard trigger (the trigger logic was computer-settable). The LAC PRETRIGGER definition was modified so that the the PRETRIGGER did not require any signal from the $p_{T}$ boards; this also implies that the EARLY PT veto was not taken into account. Only one PRETRIGGER in ten was allowed to give a trigger, thus making the trigger rate more manageable.

\subsection{Forward calorimeter}

The forward hadron calorimeter [48] is designed to cover the forward (projectile) beam-jet region, which is not covered by the liquid argon calorimeter. It can provide the capability for studying correlations between the fragmentation of the beam jet and the characteristics of the triggering collision at large $p_{T}$.

The calorimeter consists of three identical units composed of alternating layers 
of steel and acrylic scintillator, for a total of about 11 proton absorption lengths. The read out is done via $B B Q$ wavelength-shifter rods [48] that run parallel to the beam direction. A flash analog-to-digital converter (ADC) system operating at $100 \mathrm{MHz}$ is used to digitize the signals from the photomultipliers tubes coupled to each rod. The calorimeter is capable of operating at rates of up to $10 \mathrm{MHz}$. The purpose of this device is to measure the total energy and the overall $p_{T}$ of all hadrons produced at angles $<20 \mathrm{mr}$. Since the showers from the individual hadrons overlap greatly at small angles, we do not attempt to obtain individual hadron information.

\subsection{On-line monitoring and data acquisition}

The general hardware scheme for the E706 on-line system was shown in Fig. [2.3].

We use the VAXONLINE system software provided by the Data Acquisition Sofware group of the Fermilab computing department [49]. The on-line software contains programs (known as consumer processes) to produce event dumps, histograms, plots and event displays for the purpose of monitoring the experiment during data acquisition. The event rate is determined by threshold set for the $p_{T}$ trigger and it is limited by the time required by the on-line computer system to record the data. 


\section{Chapter 3}

EVENT RECONSTRUCTION

\subsection{General considerations}

This chapter describes the reconstruction package (MAGIC version 2.06) used to process the data presented in this thesis; since development work on the experiment is still in progress, this chapter is not an up-to-date description of the latest E706 off-line software.

The photon reconstruction algorithm (EMREC) is explained in detail; the track reconstruction (PLREC) and the vertex finding algorithm are also introduced. For the terminology related to the experimental apparatus used here, we refer the reader to Chapt. 2. All the plots shown in this chapter are produced using Monte Carlo generated events, unless explicitly stated to the contrary.

The E706 offline package is a library of subroutines driven by the main program MAGIC. The package is written in FORTRAN 77, makes use of the standard CERN PROGRAM LIBRARY software and it is maintained using the PATCHY [50] file management system. Since FORTRAN 77 lacks dynamic data structuring facilities, we use the CERN ZEBRA [51] data management system in the E706 software. ZEBRA allows not only a truly dynamic creation of data structures at execution time, but it also has the added advantage of providing the ability to manipulate those structures, to write them to an external storage medium, and to recover them intact. ZEBRA provides a significant extension to the power of FORTRAN, gener- 
ally at an insignificant cost in terms of execution-time overhead. The unit of storage (continuos area of storage of a given length) is called a bank. The basic connections of data structures are the links (structural and reference links). Data banks can be organized in linear structures or in tree structures.

\subsection{Shower generation}

We used two different Monte Carlo packages during the development of EMREC:

1. A package using a shower parametrization derived from the EGS (ElectronGamma-Shower) program [52], assuming a simplified geometry for the calorimeter. We made use of it to simulate in full detail the development of electromagnetic showers. The EGS system of computer codes is a general purpose package for the Monte Carlo simulation of the coupled transport of electrons and photons in an arbitrary geometry.

2. A program (E706 GEANT) using the GEANT [53] package, which reproduces in detail the geometry of the detector. In GEANT we may generate data according to a shower parametrization, or simulate in full detail the development of the shower.

The results presented in this chapter were obtained using the first package described above. E706 GEANT, with the shower parametrization option, was used for all the other Monte Carlo calculations shown in this thesis.

We will now describe in detail the shower parametrization based on the EGS program; the parametrization used in E706 GEANT is conceptually similar. The energy deposited by a photon in the liquid argon of the EMLAC is obtained from a shower parametrization based on EGS:

$$
P_{i}=E F_{i}(x)
$$

where $P_{i}$ is the signal in strip $i, E$ is the incident energy of the showering particle, and $F_{i}(x)$ is the fraction of energy that is deposited in strip $i ; x$ is the transverse distance of the center of the strip $i$ from the center of the shower. The formula used for the differential form of the shower shape is

$$
F(x)=A_{1} e^{-x^{2} / 2 s^{2}}+A_{2} e^{-a x}+A_{3} e^{-b x}
$$


Table 3.1: Shower shape parameters

\begin{tabular}{|c|c|c|c|c|c|c|}
\hline Parameters & $A_{1}$ & $A_{2}$ & $A_{3}$ & $s$ & $a$ & $b$ \\
\hline front section & 3.2 & 1.3 & 0.05 & 0.14 & 3.2 & 0.86 \\
\hline back section & 1.5 & 0.65 & 0.08 & 0.22 & 1.5 & 0.52 \\
\hline
\end{tabular}

The values of the parameters are listed in Table [3.1] (values are given in inches and $F(x)$ is normalized to unity).

The shower shapes are found to be essentially energy independent. The distribution in the fraction of the total energy that is deposited in the back section of the EMLAC $(\epsilon=E b a c k / E)$ was also parametrized using EGS:

$$
f(\epsilon)=B_{1} \exp \left(\epsilon-\epsilon_{1}\right)^{2} / 2 s_{1}^{2}+B_{2} \exp \left(\epsilon-\epsilon_{2}\right)^{2} / 2 s_{2}^{2}
$$

At $100 \mathrm{GeV} B_{1}=10.7, \epsilon_{1}=0.45, s_{1}=0.045, B_{2}=34.6, \epsilon_{2}=0.30, s_{2}=0.041$. The distribution function $f(\epsilon)$ increases approximately as the logarithm of E. $f(\epsilon)$ was normalized to unity.

In generating showers, losses and discontinuities at the boundaries between the inner $\phi$ and the outer $\phi$ strips and between the octant boundaries were taken into account.

In simulating the detector resolution, the pulse heights were smeared strip by strip according to the effective detector resolution:

$$
\sigma_{E}=\sqrt{(0.1)^{2}+(0.14)^{2} E}
$$

where the first term represents the $0.1 \mathrm{GeV}$ incoherent electronic noise per channel, and the second term is due to fluctuations in the sampling of the shower ( $\mathrm{E}$ is expressed in $\mathrm{GeV}$ ). Preliminary results from calibration runs show that the detector resolution is reasonably consistent with our assumptions.

\subsection{Shower reconstruction}

We used two different algorithms to reconstruct showers. 
1. In the first one (known as "front/back" reconstruction) shower positions and energies are reconstructed independently in each view (left $r$ and right $r$, relative to the centers of quadrants, and inner and outer $\phi$ ), for each quadrant separately for the front and back sections. Showers in the $\mathrm{r}$ and $\phi$ views are correlated by matching their energy quadrant by quadrant; photons in the front and back sections are then combined.

2. In the second one (known as "sum" reconstruction) the pulse height for each strip in the front section is added to the pulse height for the corresponding strip in the back section; this procedure avoids the need of combining front and back photons and appears to give a better energy determination.

Both reconstruction algorithms were used on the data presented in this thesis. The results described in this chapter were obtained using the front/back reconstruction; however, the final results were obtained using the sum reconstruction.

For historical reasons, we use the following (rather misleading) terminology: a "gamma" is a reconstructed shower in one view ( $\mathrm{r}$ or $\phi$ ), and can be thought of as a photon candidate that becomes a true photon after it is correlated with a gamma in the orthogonal view.

The overall photon reconstruction flow proceeds independently for each quadrant in the following sequential steps:

1. "Group" and "Peak" finding in a given view.

2. "Shoulder" finding in a peak.

3. Splitting of peaks inside a group to find all gammas.

4. Correlation of gammas between $\mathbf{r}$ and $\phi$ views to find photons.

5. Matching of front and back photons (only for the front/back reconstruction).

These procedures will now be described in more detail. 


\subsection{Groups and peaks}

Each quadrant is divided into 4 views: left $r$, right $r$, inner and outer $\phi$ consisting of 256, 256, 96 and 192 channels, respectively. The program scans the input pulseheight data bank for groups of contiguous channels that contain signals above a certain threshold, which is set equal to the zero suppression threshold (see Sect. [4.4]). This consecutive group of channels is called a "group" if it satisfies the following criteria:

1. There are at least 3 strips above threshold for left $r$, right $r$ or inner $\phi, 2$ for outer $\phi$ (the outer $\phi$ strips are wider).

2. The sum of the neighboring signals must be greater than $0.75 \mathrm{GeV}$.

3. The average signal in the group must be larger than $0.15 \mathrm{GeV}$.

4. The largest pulse height in the group must be greater than $0.3 \mathrm{GeV}$.

Groups can consist of single isolated peaks, or two or more peaks with the minimum between peaks above some preset threshold (Fig. 3.1). Each group is scanned for local peaks and valleys. A peak is considered significant if the difference between peak and valley energies, divided by the expected pulse height fluctuation in the peak $\sigma_{P}=\sqrt{(0.1)^{2}+(0.14)^{2} P}$ (where $P$ is energy in the peak and $\mathrm{GeV}$ units are used) is larger then 2.5 .

\subsection{Shoulders}

At high laboratory energies $(E>150 \mathrm{GeV}$ ), a sizeable fraction of the decay photons from $\pi^{0}$ 's start to coalesce in $\mathrm{r}$ and/or $\phi$ views. At $150 \mathrm{GeV}$ the minimum separation between the two photons at the LAC (for symmetric $\pi^{0}$ 's decay) is $1.6 \mathrm{~cm}$; for highly asymmetric decays, the low energy photon can appear as a small secondary maximum (shoulder) on the primary peak (Fig. [3.1]c). The usual peak finding algorithm generally does not reconstruct the two energy depositions as separate peaks, because it usually cannot find any significant local maximum for the lower 
Figure 3.1: Some representative "groups" of showers (the fit to the pulse height spectra is shown): a) Single peak group b) Double peak group c) Single peak group with a shoulder
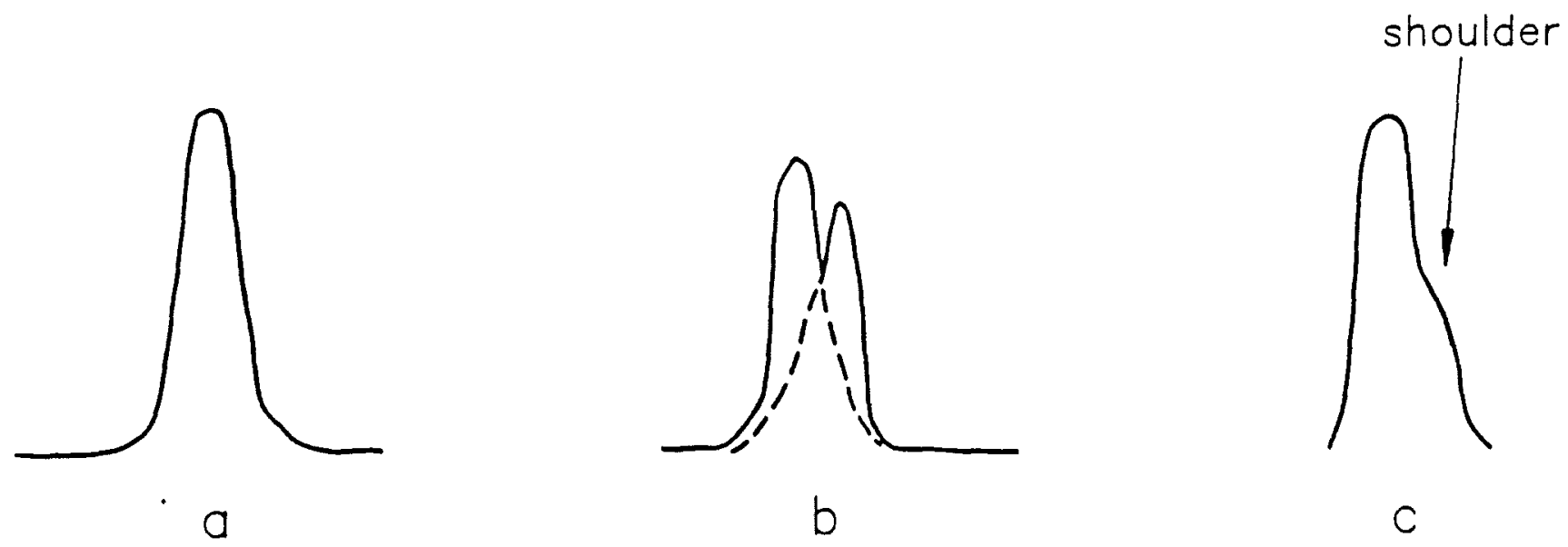
energy shower. However, the shoulder-finding algorithm attempts to separate such peaks.

This algorithm, which is not directly relevant for low $p_{T} \pi^{0}$ 's, searches for a local maximum in the logarithmic derivative of the energy difference between adjacent strips. Here, again, a shoulder is considered significant if the difference between shoulder and valley signals, divided by the expected fluctuation in the shoulder, is larger than some prescribed value. Shoulders in strips adjacent to the primary peak are not allowed because, whenever the center of shower is between two strip, a single shower can appear as a peak with a shoulder next to it. We search for shoulders only in single-peak groups, and, to avoid dealing with very ambigous (and difficult) configurations, only one shoulder is allowed per peak. Once a shoulder is found, it is fed into the splitting routine to separate it from the primary peak.

The program is designed to minimize the generation of artificial shoulders; in fact, when the program was run on 1000 single-photons of 5,7 , and $10 \mathrm{GeV} / \mathrm{c} p_{T}$, no shoulders were found. The shoulder finding algorithm was also run on generated single $\pi^{0}$ 's of 5,7 , and $10 \mathrm{GeV} / \mathrm{c} p_{T}$, and an additional $1 \%, 3 \%$ and $6 \% \pi^{0} \rightarrow \gamma \gamma$ events were found at these energies, respectively. This algorithm is, of course, also capable of separating two single photons that have accidentally merged and appear as one peak with a shoulder next to it.

\subsection{Splitting of peaks}

The shower positions $r$ and $\phi$ are calculated as: $r=r_{0}+\delta r, \phi=\phi_{0}+\delta \phi$ where $r$ is the position of the shower, $r_{0}$ is the position of the center of the peak channel, and $\delta \boldsymbol{r}($ or $\delta \phi)$ is a small correction given by:

$$
\delta r=\alpha A+\beta A^{3}
$$

where $A=\left(P_{3}-P_{1}\right) / P_{2}$, and $\alpha$ and $\beta$ are fit parameters. The parameters are obtained from a fit to the distribution of $\delta r$ as a function of $A$ for Monte Carlo generated showers. The values of A and B are given in Table [3.2]. This method is effective because electromagnetic showers have well known shape characteristics.

The energy is calculated by summing the pulse heights of a number of strips $N\left(N=N_{0}+E_{3} 0.2\right)$ in the group around the peak strip $\left(N_{0}=10\right.$ for the front 
Table 3.2: Parameters for the determination of the shower position

\begin{tabular}{|c|c|c|}
\hline Parameters & $\alpha$ & $\beta$ \\
\hline$\Gamma$ views & 0.39 & -0.002 \\
\hline inner $\phi$ view & 0.40 & -0.001 \\
\hline outer $\phi$ view & 0.60 & -0.001 \\
\hline
\end{tabular}

section, $N_{0}=15$ for the back section as well as for the sum reconstruction, and $E_{3}$ is the sum of the pulse heights in the three central strips expressed in $\mathrm{GeV}$ ). The number $N$ increases slightly with energy because the number of strips above threshold increases with energy.

When there are multiple peaks in a group (overlapping showers), the peaks are first split, and the energies and positions of the split peaks are calculated using the corrected pulse array. The splitting is done using the $\chi^{2}$ minimization algorithm described below.

If $P_{i}$ is the pulse height in strip $i, F_{i j}$ is the fraction of energy in strip $i$ due to shower $j$ and $E_{j}$ is the true energy of shower $j$, then we can write the $\chi^{2}$ function to be minimized as follows:

$$
\chi^{2}=\sum_{i}\left(P_{i}-\sum_{j} E_{j} F_{i j}\right)^{2}
$$

Setting

$$
\frac{\partial \chi^{2}}{\partial E_{k}}=0
$$

requires for each $k$

$$
\sum_{i}\left(P_{i}-\sum_{j} E_{j} F_{i j}\right) F_{i k}=0
$$

or

$$
\sum_{j} E_{j} \sum_{i} F_{i j} F_{i k}=\sum_{i} P_{i} F_{i k}
$$

Defining: $A_{j k}=\sum_{i} F_{i j} F_{i k}=A_{k j}$ and $B_{k}=\sum_{i} P_{i} F_{i k}$ we can write:

$$
B_{k}=\sum_{j} E_{j} A_{j k}
$$


which gives:

$$
E_{j}=A_{j k}^{-1} B_{k}
$$

The splitting of overlapping showers is then performed by inverting a symmetric matrix of dimension $M$ (where $M$ is the number of showers); the inversion provides all the individual energies $E_{j}, j=1, \ldots, M$. The true signal due to any individual shower $j$, can be calculated by subtracting the contributions of neighboring showers from an observed pulse height:

$$
P_{i j}^{t r u e}=P_{i}-\sum_{k} E_{k} F_{i k},(k \neq j)
$$

The position of a shower can also be calculated using these true signals for the three central strips. To correct for energy shifts due to the new shower positions, the process is iterated, and the solution with the lowest $\chi^{2}$ is then accepted as best.

\subsection{Correlation of $\mathrm{r}$ and $\phi$ views}

Showers reconstructed in $\mathrm{r}$ and $\phi$ views are correlated to match their energies. The quantity

$$
\Delta C=\left(\left|E_{r}-E_{\phi}\right|\right) / \sigma_{C}
$$

where $\sigma_{C}=\sqrt{(n 0.1)^{2}+(0.14)^{2}\left(E_{r}+E_{\phi}\right)}$, is the fluctuation expected in $E_{r}-E_{\phi}(n$ is the number of strips used in the energy calculation and all energies are expressed in $\mathrm{GeV}$ ) is the quality factor used in the correlation.

The correlation algorithm proceeds in the following steps:

1. If there are any showers which span the inner/outer $\phi$ boundary or the octant boundaries, we attempt to correlate them first, with the requirement that the shower positions on either side of the the boundary match within a tolerance $\Delta d$. There are three iterations; $\Delta d$ is initially $1 \mathrm{~cm}$ for the front section and the sum reconstructor and $2 \mathrm{~cm}$ for the back section, then $\Delta d$ is incremented by its initial value in the two additional iterations. For each of these iterations in $\Delta d$ three iterations in $\Delta C=1$ are allowed, starting with $\Delta C=1$ and incrementing it each time by 1 . 
2. The remaining showers are correlated separately in each correlation region (inner $\phi$-left $\mathrm{r}$, outer $\phi$-left $\mathrm{r}$, inner $\phi$-right $\mathrm{r}$ and outer $\phi$-right $\mathrm{r}$ ). Seven iterations are allowed, starting with $\Delta C=1$, and incrementing it each time by 1 ; the procedure is stopped if the maximum uncorrelated energy per view is smaller than some prescribed value.

For each of the previous two steps, the following operations are performed:

1. All $\mathrm{r}$ and $\phi$ combinations are checked for a possible correlation, and the best match is accepted if it passes the $\Delta C$ cut (and the $\Delta d$ cut for a correlation at a boundary).

2. Showers that coalesce in one view, but are separated in the other ( $1 \mathrm{r}$ and $2 \phi$, or $2 \mathrm{r}$ and $1 \phi$ correlations) are split into two, with the energy being assigned from the view that has an obvious split. A fit, with several iterations, is then performed to obtain the best split.

Figure [3.2] shows the energy correlation between $E_{r}$ and $E_{\phi}$ for $7 \mathrm{GeV} / \mathrm{c} p_{T}$ Monte Carlo single photons. $E_{r}-E_{\phi}$ provides a measure of the effective resolution of the LAC.

In the front/back reconstruction we finally combine photons from the front and back sections; this is not needed in the sum reconstruction. To combine front and back photons, photons found in the front section are projected to the back section, and any photons that are found within $4 \mathrm{~cm}$ of the projected center are combined with the photon in the front section. The position is determined using the front section information and the energy is obtained from a sum of front and back energies.

\subsection{Prediction of $\mathrm{r}$ from the width of the shower in $\phi$}

Since the width of a $\phi$ strip changes with $\mathbf{r}$ it is possible to predict an average value of $r$ from the width of the shower observed in the $\phi$ view. We calculate 
Figure 3.2: Energy correlation between $r$ and $\phi$ view for the front section of the $\mathrm{LAC}$ for $7 \mathrm{GeV} / \mathrm{c} p_{T}$ Monte Carlo single photons.

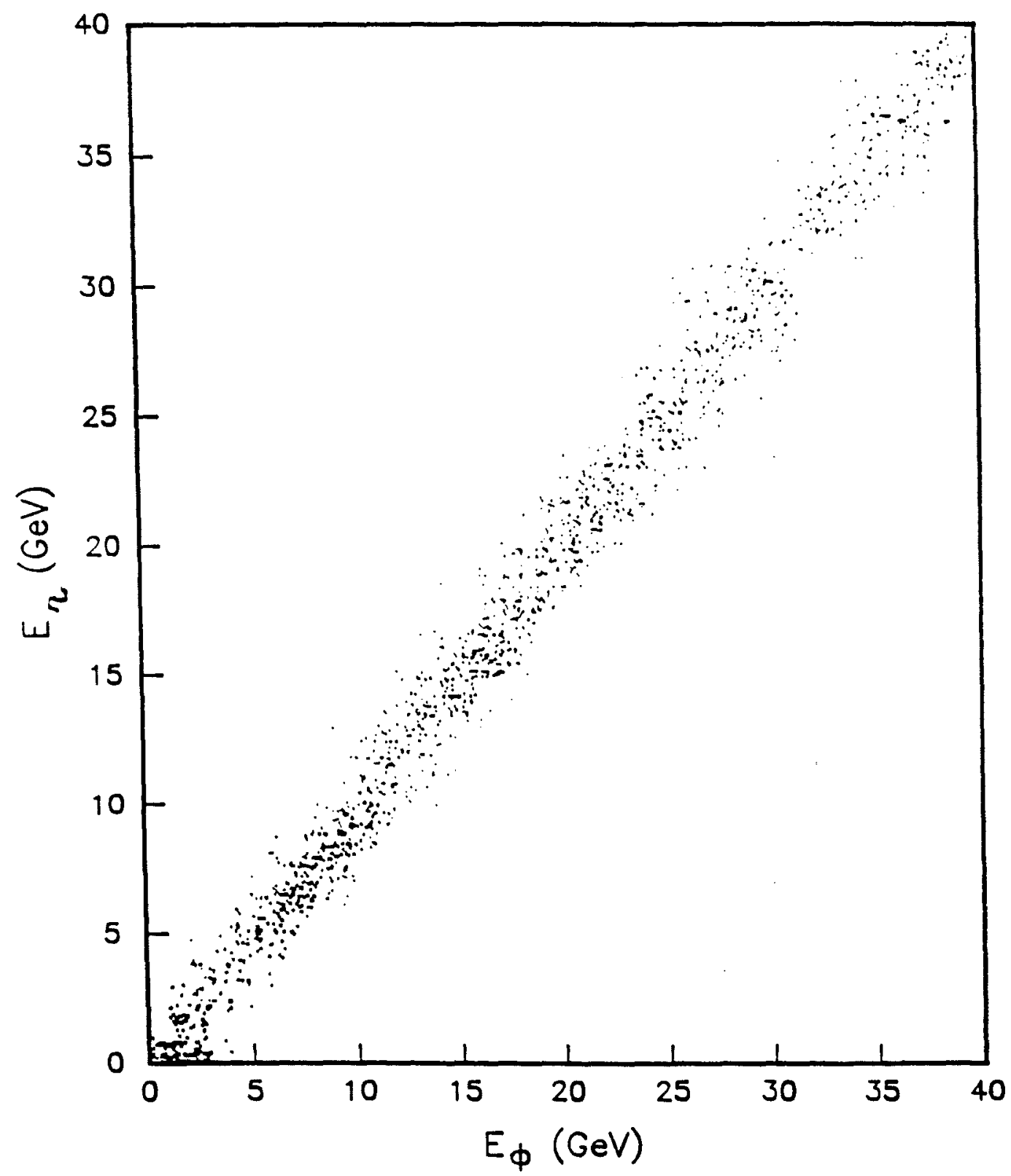


the root-mean square width in $\phi$ as follows: $\sigma_{\phi}=\sqrt{\left(\left\langle x^{2}\right\rangle-\langle x\rangle^{2}\right)}$, where $\langle x\rangle^{2}=\sum x_{i}^{2} P_{i} / \sum P_{i},\langle x\rangle=\sum x_{i} P_{i} / \sum P_{i}, x_{i}$ refers to strip numbers, and the sums are taken over the central five strips of a shower in the $\phi$ view. Figure [3.3) shows the variation of $\sigma_{\phi}$ (in units of number of strips) as a function of the radial distance $r$ for $100 \mathrm{GeV}$ Monte Carlo showers; the discontinuity at $r=40 \mathrm{~cm}$ corresponds to the inner $\phi$ /outer $\phi$ boundary, and is due to the change in width of $\phi$ strips at that radius.

The average value of $r$ for any shower can be determined by table interpolation. Figure [3.4] shows the difference between the predicted and the generated position in $r$ for single showers near $r=100 \mathrm{~cm}$, and indicates that the accuracy of the prediction is about $\pm 5 \mathrm{~cm}$ for $100 \mathrm{GeV}$ single showers. The error on the prediction is found to be dependent on $r$ and on shower energy. This predicted value of $r$ is used to impose a loose cut for the correlation of showers between the $r$ and $\phi$ views.

\subsection{Walker mass}

The width of a shower has long been known to reflect the mass of the particle that produced it. The Walker mass relation [54] can be used to calculate the mass of multiphoton showers that merge completely in both views. For a $\pi^{0}$ this relation becomes:

$$
\sigma_{\pi^{0}}^{2}=\sigma_{s}^{2}+d^{2} M_{\pi^{0}}^{2} / E_{\pi^{0}}^{2}
$$

where $\sigma_{s}$ and $\sigma_{\pi^{0}}$ are the root-mean square widths of a single photon shower and of a $\pi_{0}$ respectively, and $d$ is the distance of the detector from the interaction vertex.

Figure [3.5] shows the square of the width of the shower in $\mathrm{cm}^{2}$; the $\sigma^{2}=$ $\sigma_{r}^{2}+\sigma_{\phi}^{2}$ distribution is for all reconstructed photons from the decay of $10 \mathrm{GeV} / \mathrm{c} p_{T}$ single $\pi^{0}$ 's; the peak on the left is due to the isolated photons, whereas the peak on the right is due to merged $\pi^{0}$ 's. Although separating $\gamma$ 's from $\pi^{0}$ 's at this energy (about $200 \mathrm{GeV}$ ) is not trivial, there is, nevertheless, a clear valley between the two peaks. $\quad M_{\pi^{0}}^{2}$, calculated using the above formula for $10 \mathrm{GeV} / \mathrm{c} p_{T} \pi_{0}$ 's, with $\sigma^{2}>1.0$, is shown in Fig. [3.6]. The separation $\gamma / \pi^{0}$ is remarkably good: a broad $\pi^{0}$ peak is observed around $0.140 \mathrm{GeV} / \mathrm{c}^{2}$; the lower peak is caused by isolated single photons. We can define as $\pi^{0}$ 's those showers whose Walker mass is between 0.085 and $0.185 \mathrm{GeV} / \mathrm{c}^{2}$. To ascertain the fraction of single photons that would pass this 

Figure 3.3: $\sigma_{b}$ (in units of number of strips) as a function of radial distance for 100
GeV Monte Carlo generated photon showrers.

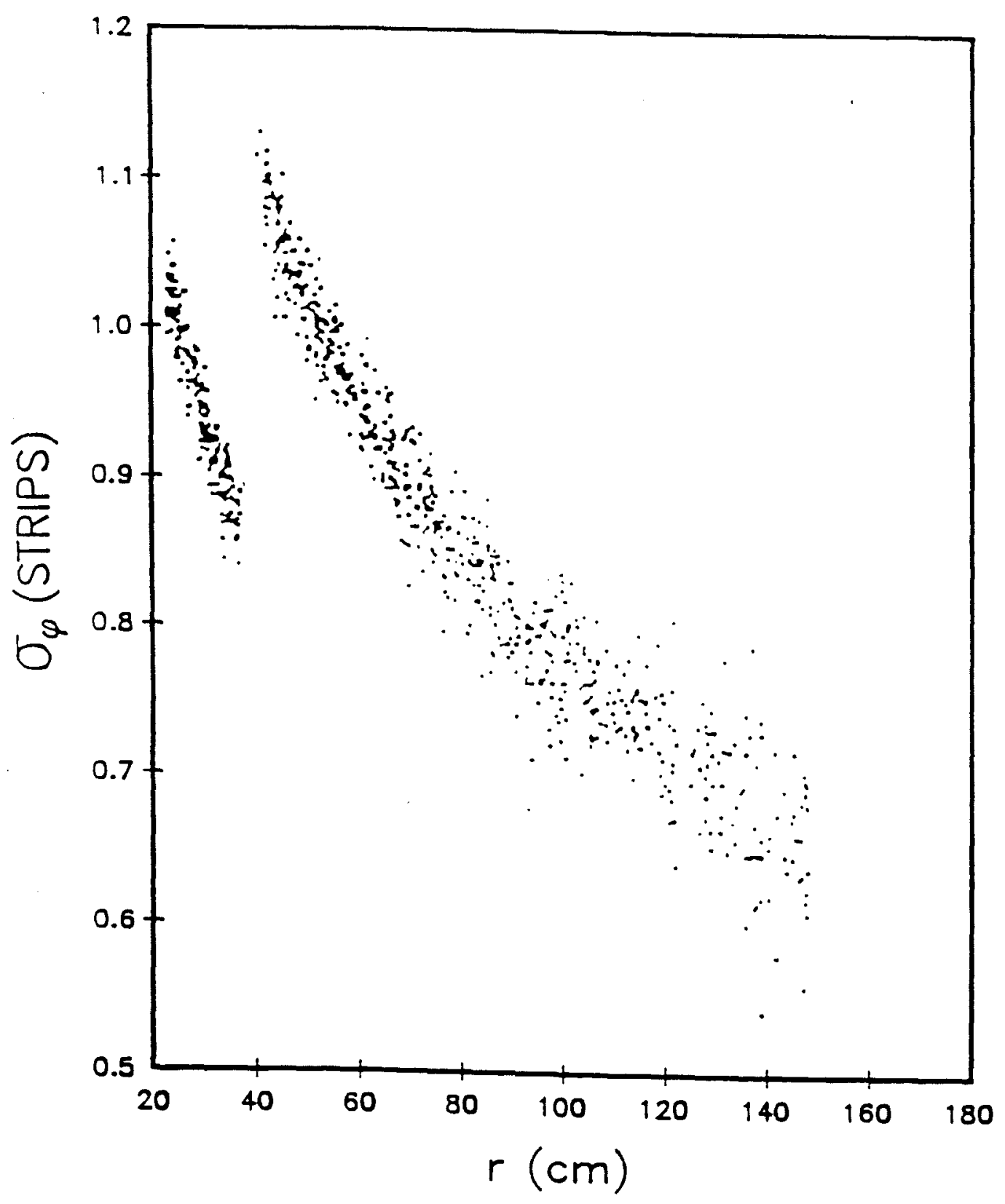


Figure 3.t: Resolution in r prediction from shower width in ofor 100 (iel photons generated near $r=100 \mathrm{~cm}$

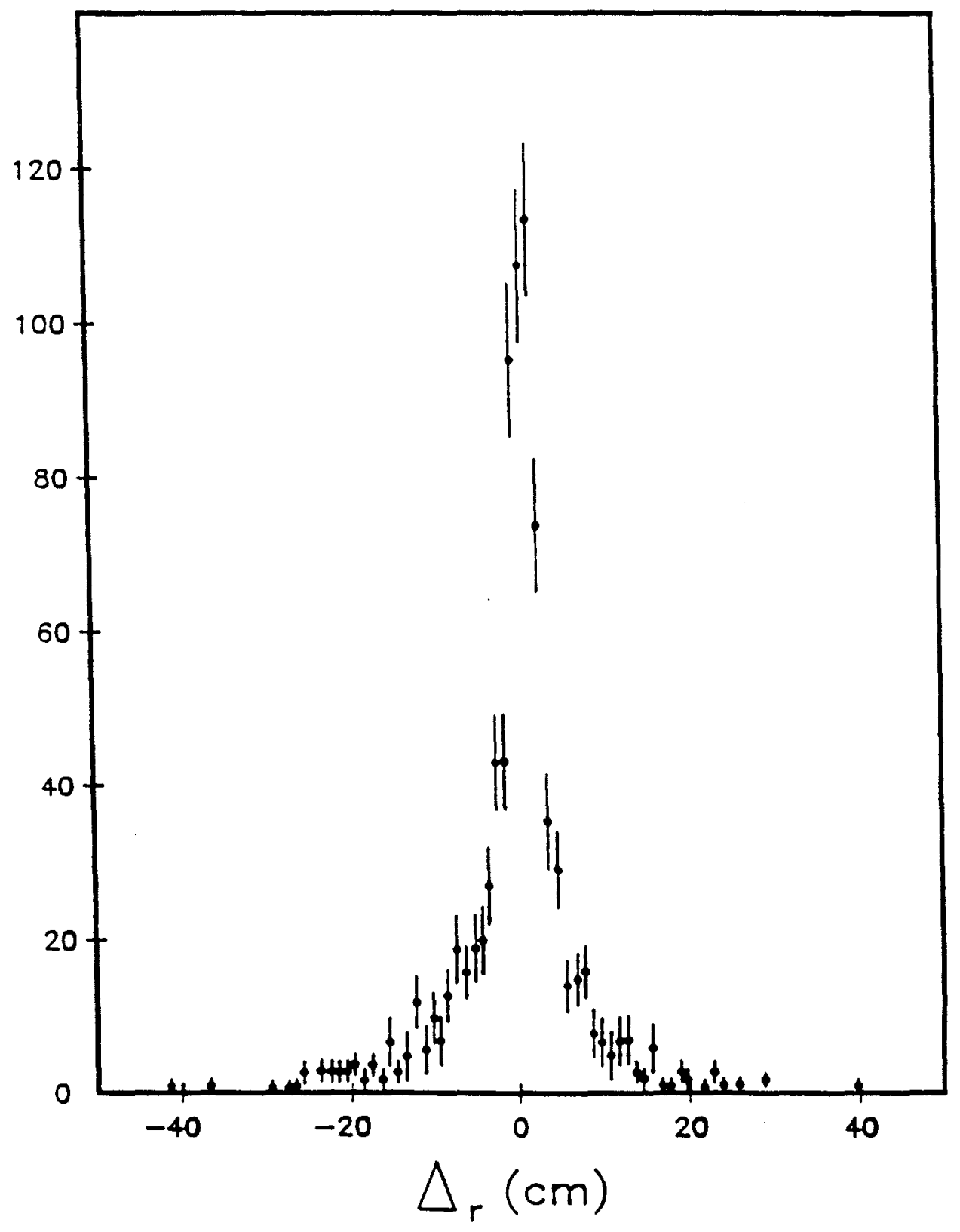



Figure 3.5: Distribution of of the square of the width of showers for $10 \mathrm{GeV} / \mathrm{c} p_{T}$
single $\pi^{0}$ erents

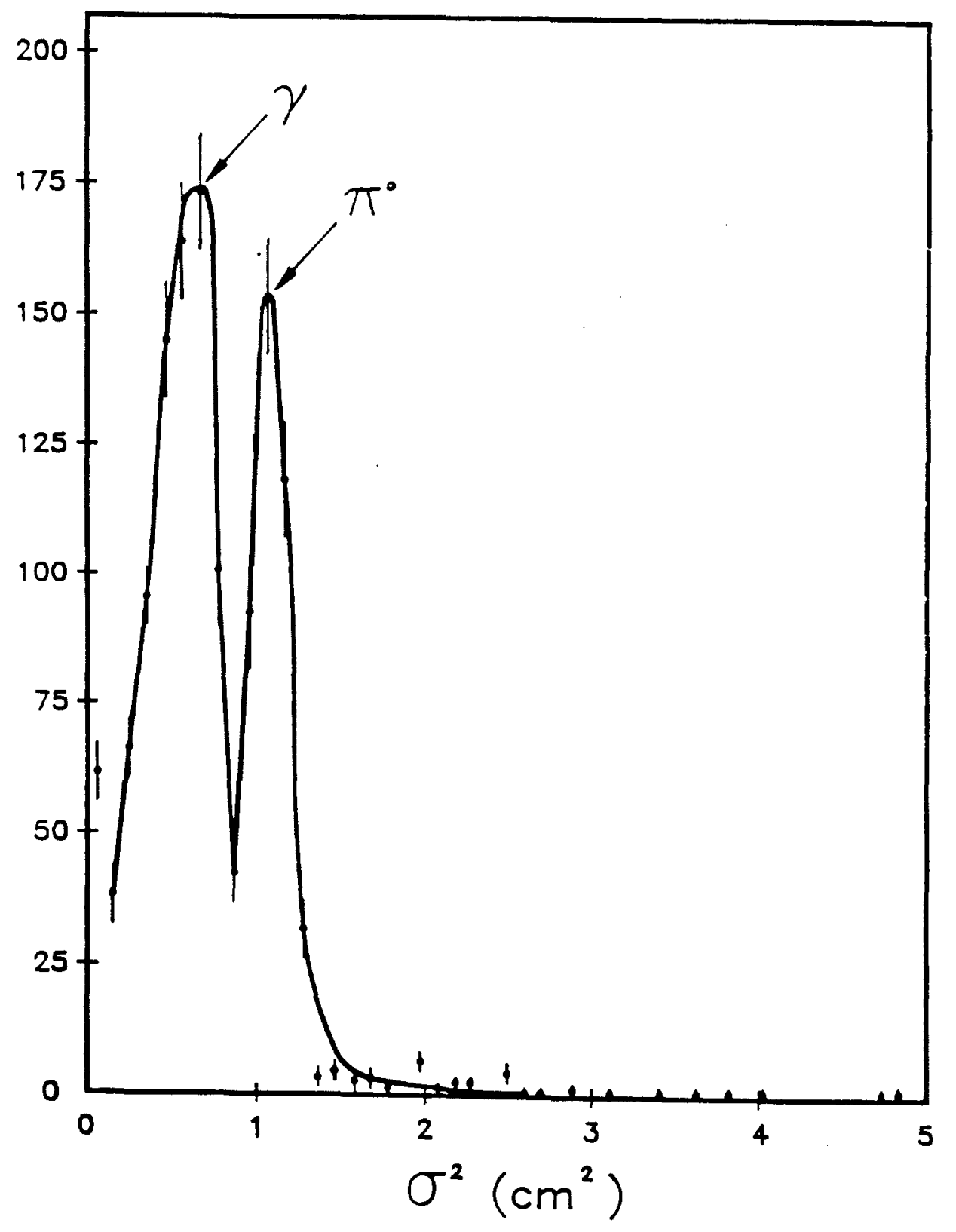

Ballocchi, Giusepp Ballocchi, 
Figure 3.6: Walker mass distribution calculated for $\sigma^{2}>1 \mathrm{~cm} \mathrm{~m}^{2}$. For single $\pi^{0}$ events with $p_{T}=10(\mathrm{reV} / \mathrm{c}$

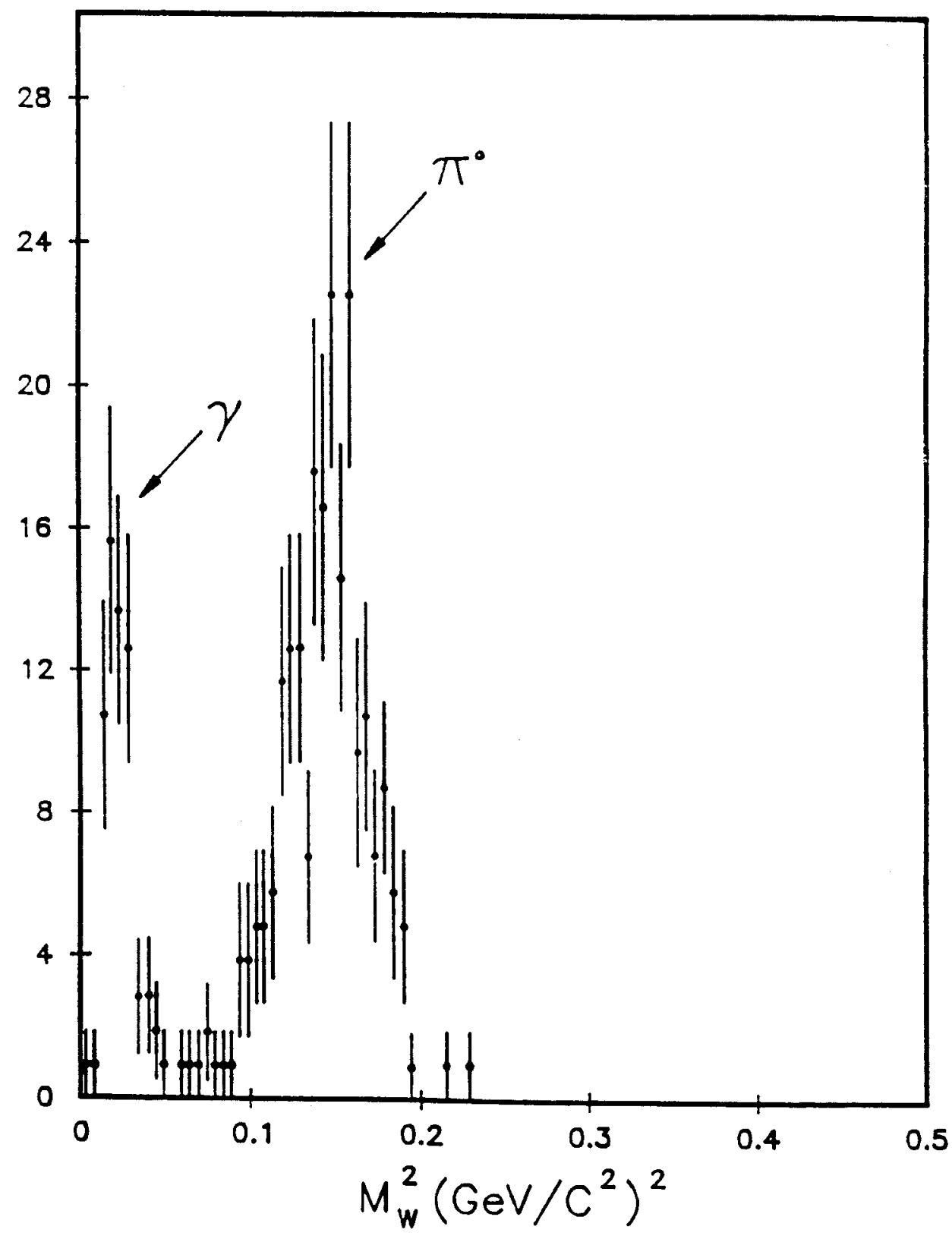


criterion, we ran the program on single photon events of 5,7 and $10 \mathrm{GeV} / \mathrm{c} p_{T}$, and conclude that the probability of labeling a photon as a $\pi^{0}$ is typically less than 1.0\%. Also for single $\pi^{0}$ of $p_{T}=5,7,10 \mathrm{GeV} / \mathrm{c}$, using the Walker mass allows to reconstruct $1 \%, 5 \%$, and $17 \%$ more $\pi^{0}$ 's, respectively.

\subsection{Testing the reconstructor using Monte Carlo events}

The following section describes the results of some detailed Monte Carlo studies of EMREC. These studies provided an early indication of the overall efficiency of the reconstruction package.

- Single photon events. Single photons of $7 \mathrm{GeV} / \mathrm{c} p_{T}$ were generated uniformly in rapidity within the acceptance of the $\mathrm{LAC}$, then reconstructed and finally compared to the generated parameters. The average position resolution in the $r$-view is $\sigma_{r} \approx 0.25 \mathrm{~mm}$ (see Fig. [3.7]); we expect the position resolution to be worse for real data, because of the shape of the real showers may vary more due to the dependence on position in the calorimeter and because of noise contributions.

The achieved energy resolution is shown in Fig. [3.8]; it depends of course on the energy smearing introduced in the Monte Carlo generator.

The efficiency for reconstructing these isolated photons is about $99 \%$. From the positions of the photon in the front and the back sections of the LAC the origin (direction) of these photons can be measured typically to an accuracy of about $5 \mathrm{mr}$.

- Single $\pi^{0}$ events. Single $\pi^{0}$ 's with fixed values of $p_{T}=5,7$ and $10 \mathrm{GeV} / \mathrm{c}$ were generated with uniform rapidity within the acceptance of the LAC; these were decayed into two photons and then reconstructed. Figure [3.9] shows the reconstructed two-photon mass, for $\pi^{0}$ 's with $p_{T}=7 \mathrm{GeV} / \mathrm{c}$; the peak is at the correct value, and $\sigma_{\pi^{0}}=7.5 \mathrm{MeV} / \mathrm{c}^{2}$.

Figure [3.10] shows the reconstruction efficiency as a function of the asymmetry variable $\mathrm{A}$ in $\pi^{0}$ 's decay $\left(A=\left(\left|E_{1}-E_{2}\right|\right) /\left(E_{1}+E_{2}\right)\right.$, where $E_{1}$ and $E_{2}$ are the energies of the decay photons). 

Figure 3.7: Radial position resolution for Monic ('arlo photons in the front section
of the FALAC:

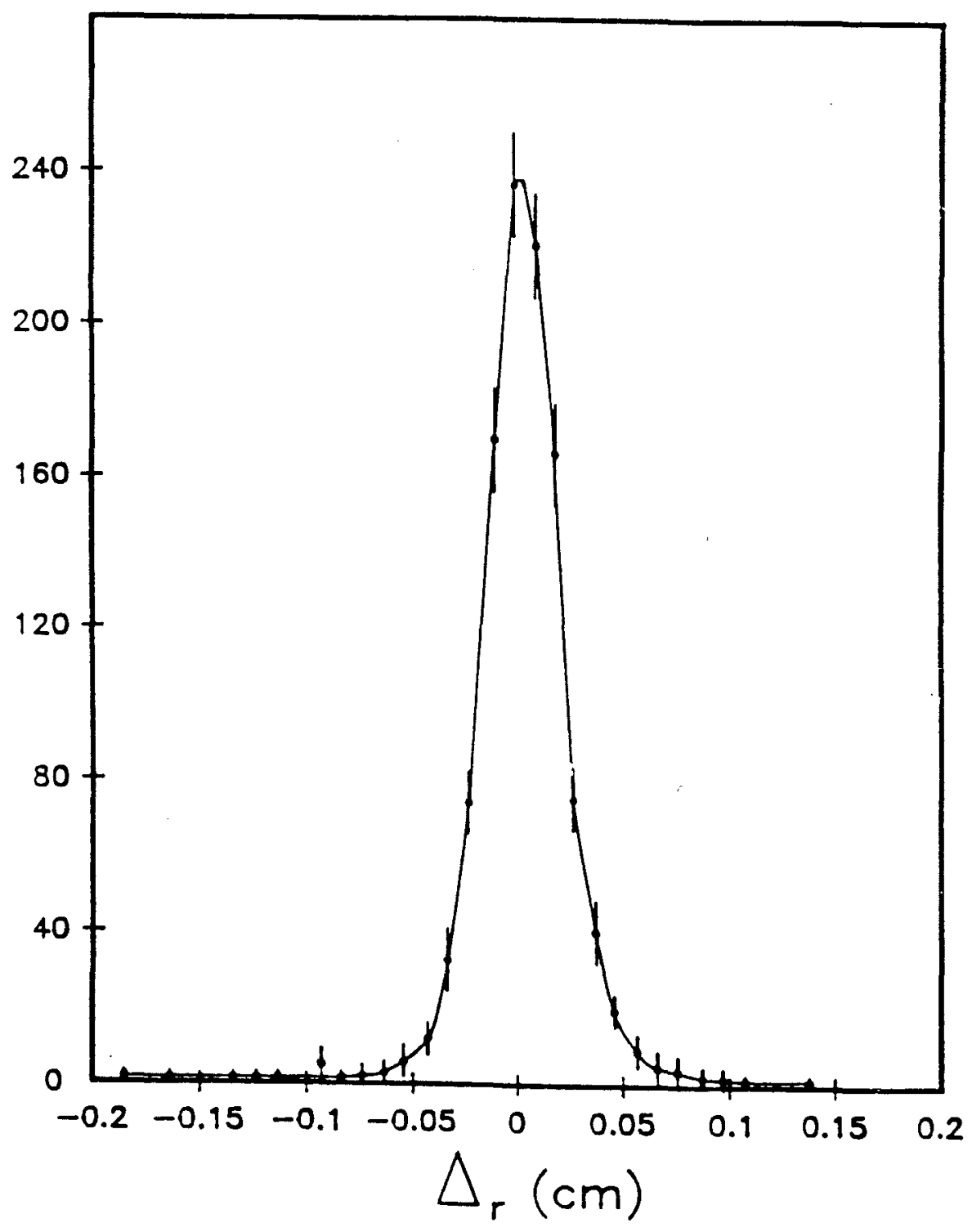


Figure 3.8: Vincrgy resolution for Monte ('arto photons

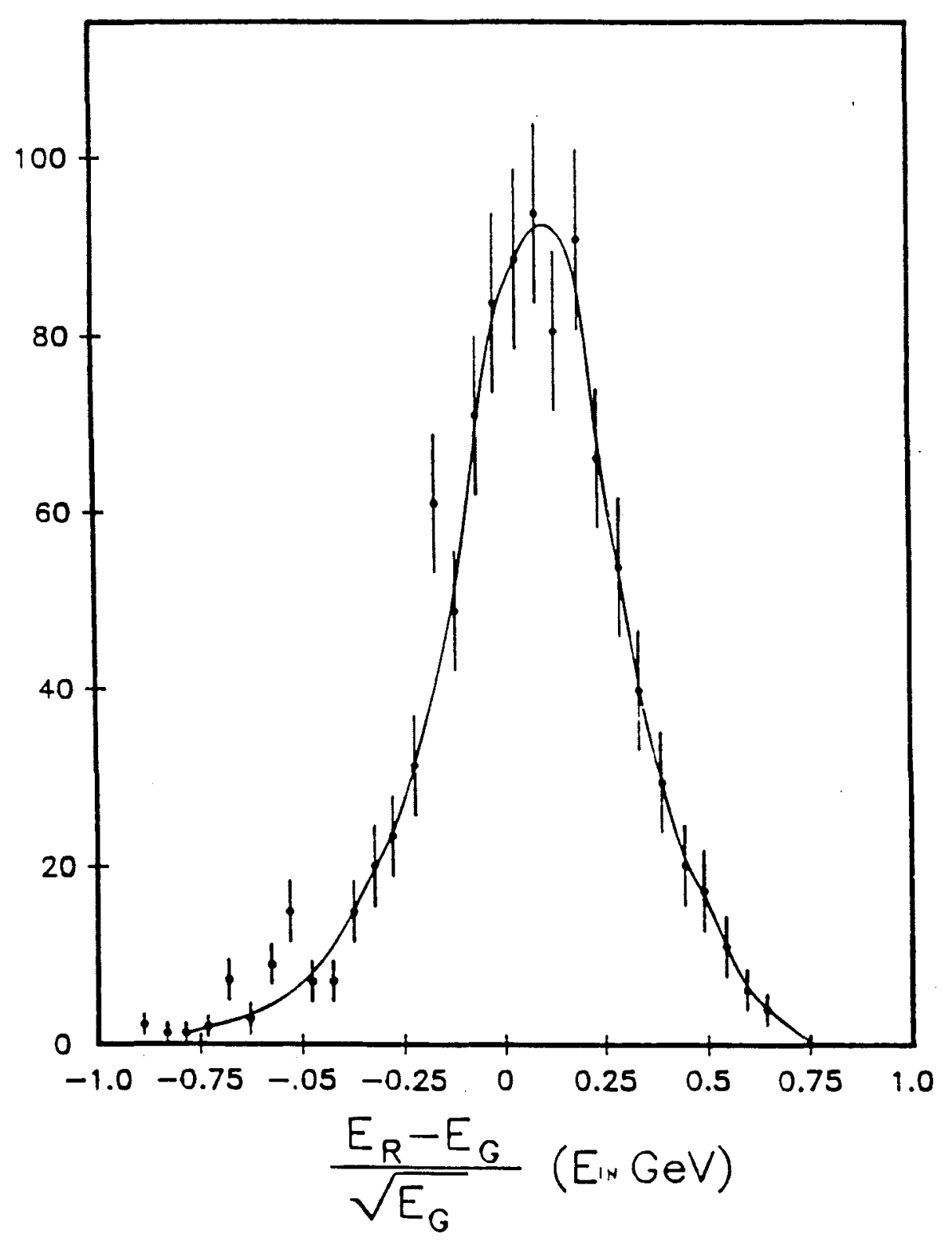


Figure 3.9: Two-photon mass distribution for $T$ ( $\mathrm{iel} / \mathrm{C}$ pre single $\pi^{0}$ events

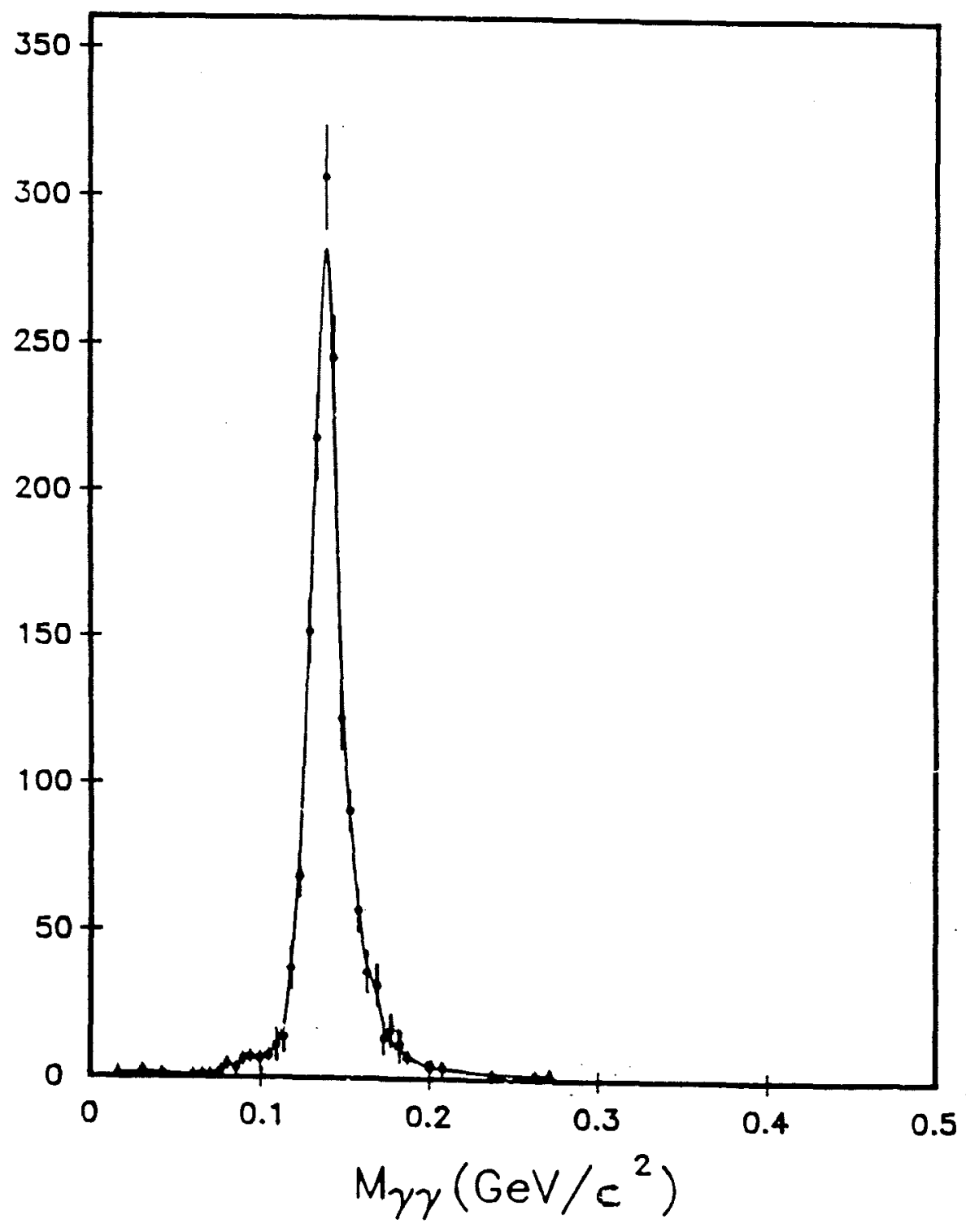


Figure 3.10: Reconstruction efficiency versus asymmetry for $7 \mathrm{GeV} / \mathrm{c} p_{T} \pi^{0}$ 's

$\omega$

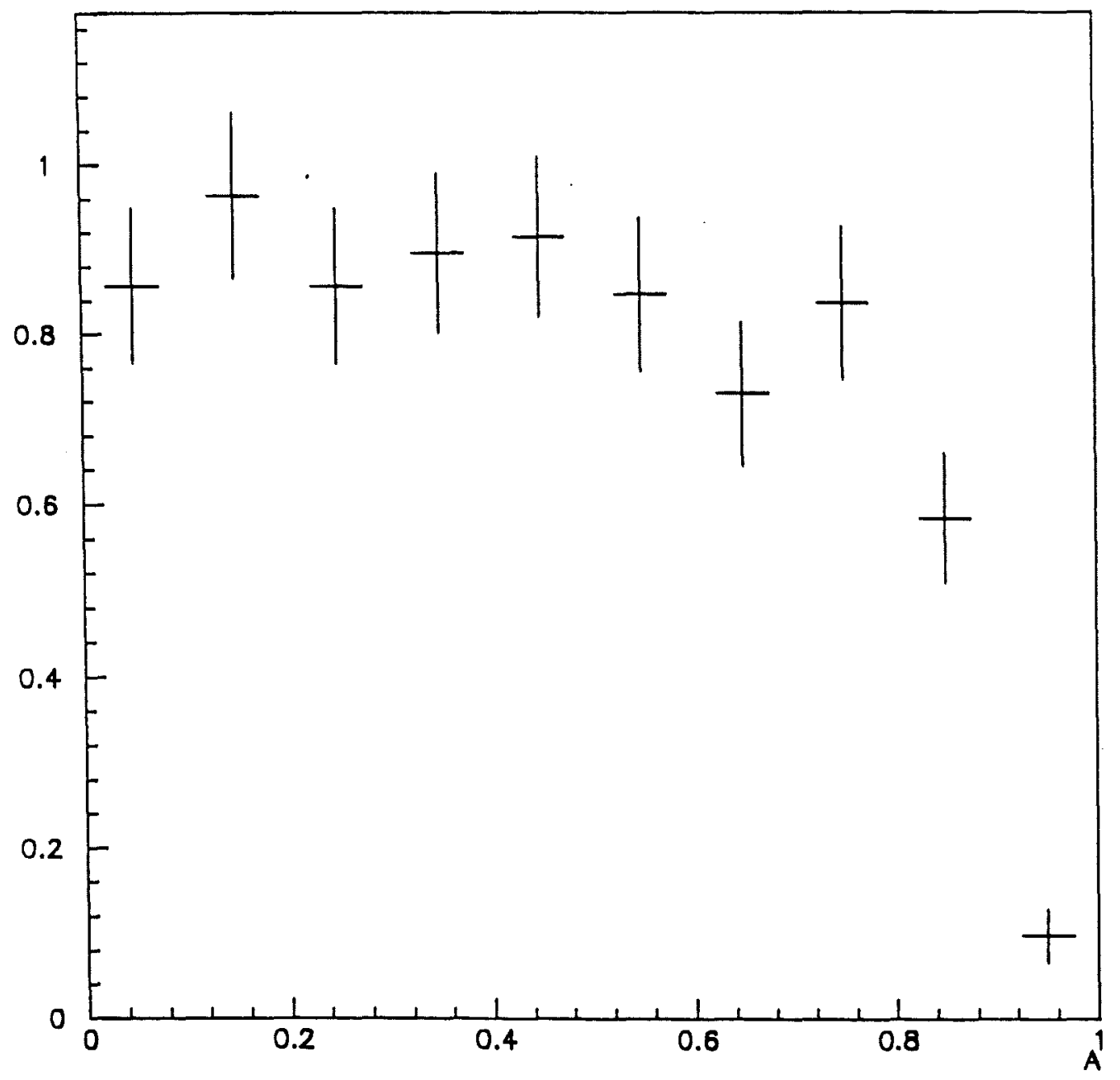


Defining as $\pi^{0}$ 's the photon-photon systems whose mass was between $85-185$ $\mathrm{MeV}$, the $\pi^{0}$ 's reconstruction efficiencies are about $95 \%, 93 \%$ and $88 \%$ for $p_{T}=5,7$ and $10 \mathrm{GeV} / \mathrm{c}$, respectively (integrated over all asymmetry values, and requiring any individual photons to have energies $E_{\gamma}>2 \mathrm{GeV}$ ).

- Multi- $\pi^{0}$ 's events. We have investigated the $\pi^{0}$ reconstruction efficiency at higher $p_{T}$ in the presence of debris from the decays of $\pi^{0}$ 's of lower $p_{T}$. We have generated events with $\pi^{0}$ 's multiplicity of $5 \pm 2$, with a Gaussian distribution (we take the integer part of the generated Gaussian random number), with one high $p_{T} \pi^{0}$ at $p_{T}$ of 5,7 or $10 \mathrm{GeV} / \mathrm{c}$. The remaining $\pi^{0}$ 's were generated assuming the $p_{T}$ distribution $d \sigma / d p_{T}=\exp \left(-1.16 p_{T}\right)\left(p_{T}\right.$ is measured in $G e V / c)$. All the $\pi^{0}$ 's are generated with uniform rapidity within the acceptance of the EMLAC. The exact form assumed for the $p_{T}$ and rapidity distribution is not important for the purpose of this investigation. The $\pi^{0}$ 's are decayed with uniform asymmetry and then reconstructed. The reconstruction efficiencies for this class of events are found to be $87 \%, 86 \%$ and $83 \%$ at 5,7 and $10 \mathrm{GeV} / \mathrm{c} p_{T}$, respectively (integrated over all asymmetry and again with $E_{\gamma}>2 \mathrm{GeV}$ ). The debris from the low $p_{T}$ causes a loss of about $5-8 \%$ of the higher $p_{T} \pi^{0}$ 's. Figure [3.11] shows the two-photon mass distribution for all reconstructed doublets in multi- $\pi^{0}$ events, in which one $\pi^{0}$ has more than $7 \mathrm{GeV} / \mathrm{c} p_{T}$.

Figure [3.12] shows the same data, but requiring that any doublet must have $p_{T}>5 \mathrm{GeV} / \mathrm{c}$. It appears that the low $p_{T}$ background has little effect on the high $p_{T}$ signal.

- ISAJET events. Using the ISAJET program [55] direct photon events of a mean $p_{T}$ of $7 \mathrm{GeV} / \mathrm{c}$ were generated according to the lowest order QCD cross section. The photon reconstruction package was then run on this class of events.

The photon and $\pi^{0}$ reconstruction efficiency for all values of $p_{T}$ (but with $E_{\gamma}>2 \mathrm{GeV}$ ) is $75 \%$ and $60 \%$, respectively. The low detection efficiencies are due to the fact that ISAJET tends to generate many low $p_{T}$ and low energy photons $/ \pi^{0}$ 's; the accepted photon $/ \pi^{0}$ energy distribution appears to be exponential in form, with a mean of about $10 / 20 \mathrm{GeV}$. Of the photons that are lost, about $75 \%$ have energy between $2-5 \mathrm{GeV}$. For $\gamma \gamma$ combinations that have $p_{T}>1.0 \mathrm{GeV} / \mathrm{c}$, the $\pi^{0}$ detection efficiency increases to $72 \%$. 


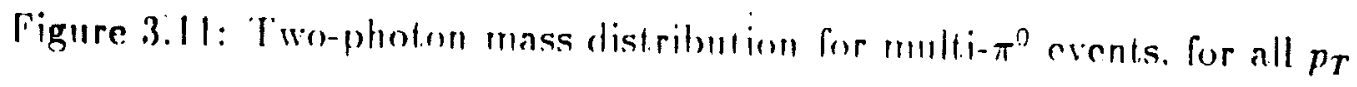

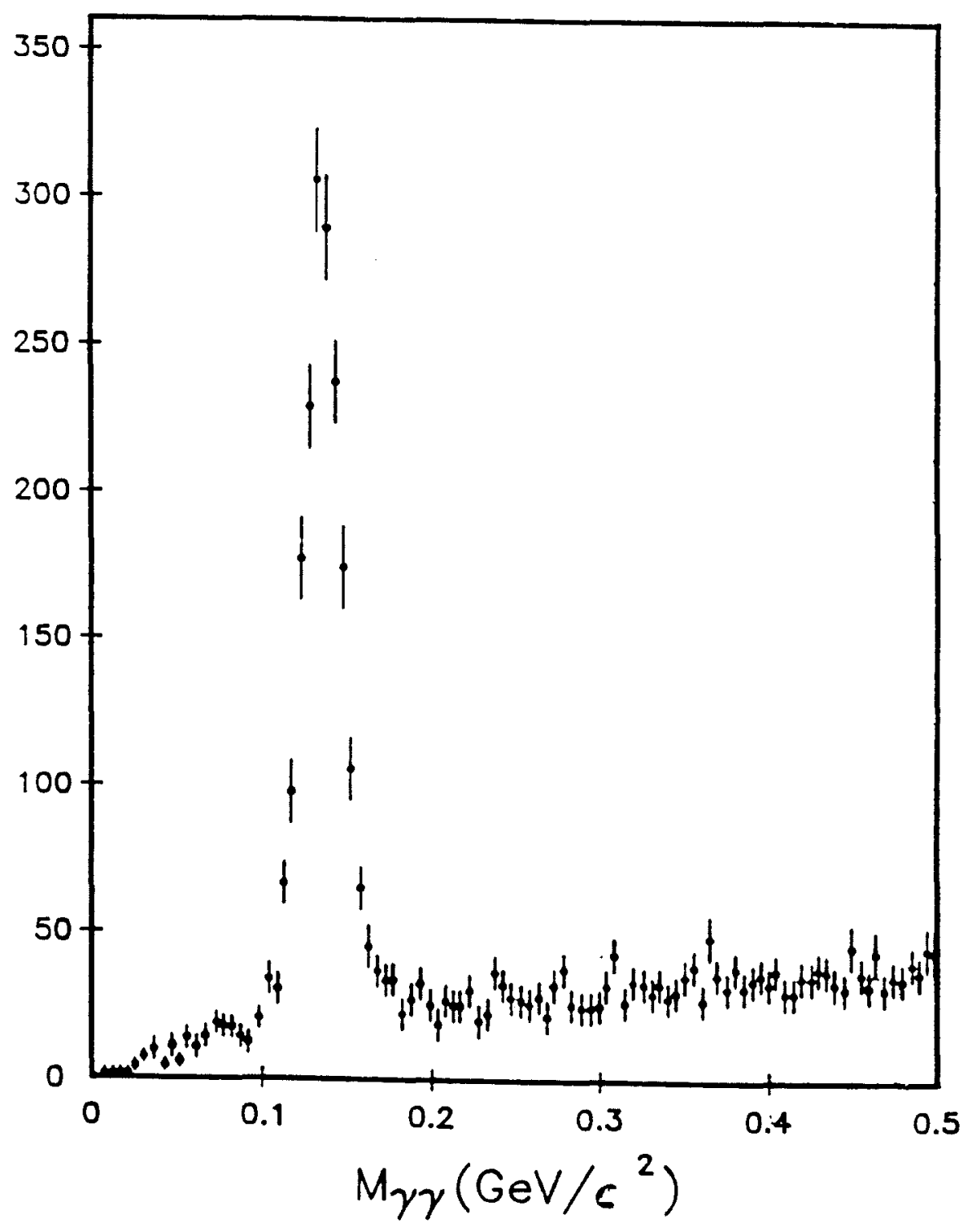


Figure 3.12: 'Two-photon mass distribution for multi- $\pi^{0}$ events, for $p_{T}>5 \mathrm{GeV} / \mathrm{c}$

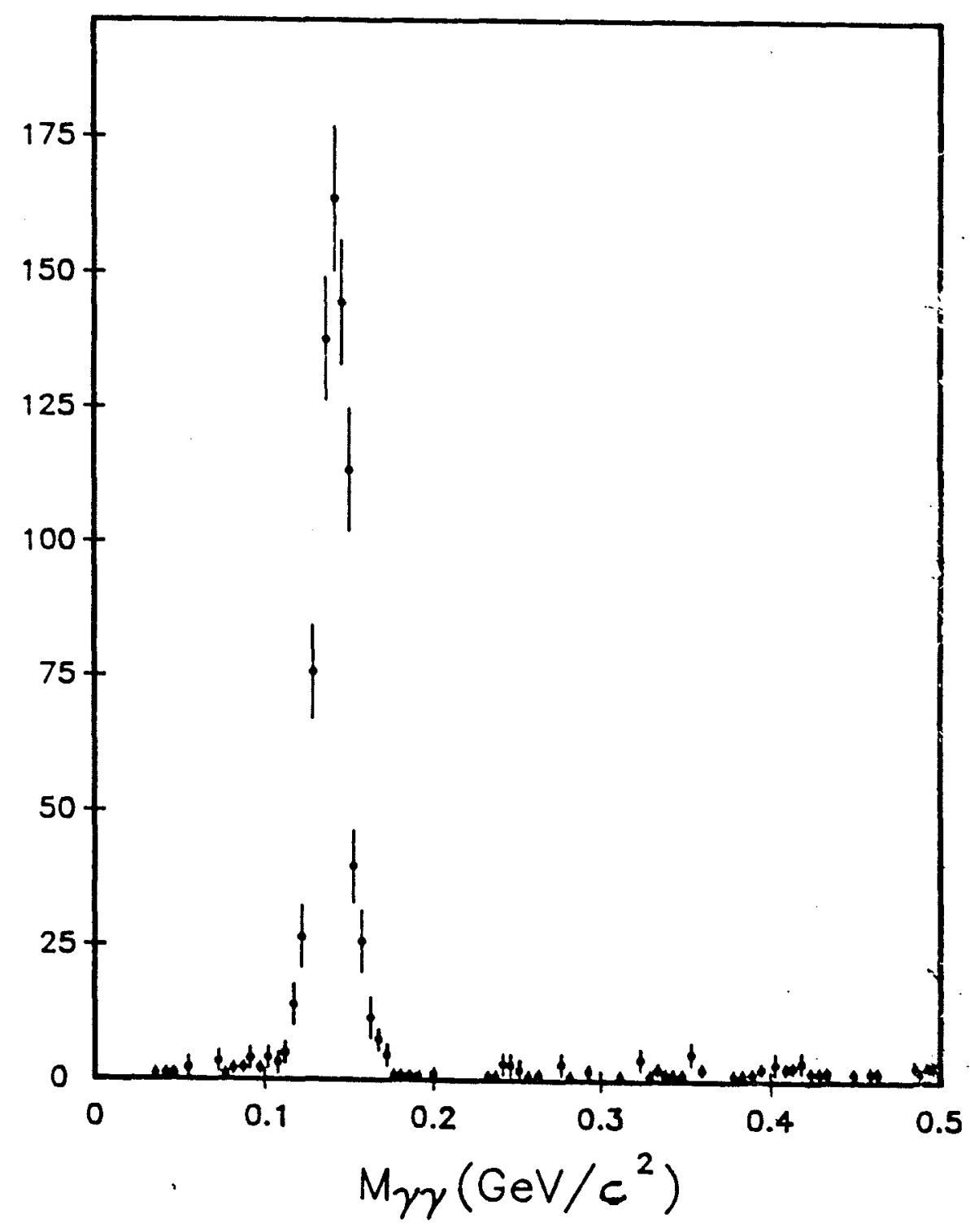




\subsection{Comments on EMREC}

1. The splitting algorithm for overlapping showers is efficient in the $\mathbf{r}$ view, but it is not entirely satisfactory in the $\phi$ views; this is because one needs to know the approximate $r$ position of the shower in order to calculate the fraction of the shower energy expected in each $\phi$ strip. The r position of the shower is estimated from the shower width in the $\phi$ view, but this estimate is subject to rather large uncertainties, especially when dealing with overlapping showers.

2. Below about $140 \mathrm{GeV}$ there are only few coalescing $\pi^{0}$ 's; coalescing starts typically above that energy. The Walker mass method proves to be the only tool that can be used to identify the high $p_{T} \pi^{0}$ 's that coalesce in both views. The Walker mass methods provides the means for tagging $72 \%$ of all $\pi^{0}$ 's that would have been otherwise lost.

3. The loss of $\pi^{0}$ 's below $p_{T} \approx 7 \mathrm{GeV} / \mathrm{c}$ is due to highly asymmetric decays, in which one photon has very low energy; at high $p_{T}$ values $(>7 \mathrm{GeV} / \mathrm{c})$, the loss is due to both highly asymmetric decay and to coalescence. In summary, the results of the last two sections indicate that the LAC, at least as far as design is concerned, is capable of separating $\gamma^{\prime}$ 's from $\pi^{0}$ 's over a large range of energies with excellent efficiency, using the EMREC program.

\subsection{Track reconstruction and vertex determina- tion}

The essential steps in the track finding algorithm are:

1. View track reconstruction. For each of the 2 views in the SSD's $(x, y)$, and for each of the four views in the MWPC's, a straight line is formed for each pair of hits in two seed planes. The straight line is then interpolated/extrapolated to the other two planes; if hits are found within a certain distance, a least square fit is made using the the found hits.

2. Space track reconstruction. Each pair of tracks in any two MWPC views is considered; hits from other views are taken into account only if they fall 
within a certain distance of the position of the projected track; if the resulting $\chi^{2}$ and number of hits are acceptable, a space track is defined. Cuts are made to reject the tracks which share many hits (to avoid multiple reconstruction of the same track).

3. Linking of upstream and downstream tracks. Each downstream space track is extrapolated to the center of the magnet and a best link is found to an upstream track by finding the closest match in the $\mathrm{x}$ and $\mathrm{y}$ views.

To define a vertex in a view of the SSD's at least three 4-hit tracks are required. If three or more 4-hit linked tracks are present in the event, the vertex determination is based on those tracks, otherwise it is based on all the 4-hit tracks, even if they were not linked downstream.

A $\chi^{2}$ is formed for the hypothesis that the tracks come from a common vertex; the position of the vertex is determined by minimizing the $\chi^{2}$ with respect to the vertex coordinates; this minimization is performed with a matrix inversion. Every track with an impact parameter (distance to the vertex) larger than $50 \mu$ is rejected and the equation for the vertex position is solved again, until the average impact parameter becomes less than $20 \mu$, or the worst impact parameter is less than $50 \mu$.

After finding a vertex in each view, if the difference in the $z$ position of the $x$ and $y$ vertices is less than $3 \mathrm{~mm}$, the weighted average of the $z$-coordinate defines the final matched vertex.

The momentum of the tracks is determined from the magnetic field map after the vertex has been found. 


\section{Chapter 4}

\section{DATA ANALYSIS}

\subsection{General considerations}

While previous chapters often dealt with the experiment as a whole, this chapter describes the procedures used for the analysis of the data in this thesis; the corrections and the cuts used to define the relevant signal are discussed in detail. The data mentioned here were taken with a dedicated interaction trigger. We wrote data summary tapes (DST's) which contain the positions and energies of the reconstructed photons, other relevant information concerning the showers in the electromagnetic calorimeter, the charged-track information from the MWPC's and from the SSD's and the data from the discrete logic. In order to save space on the summary tapes, any event with less than two showers in the EMLAC was rejected, since it could not yield a $\pi^{0}$; many events were rejected in this fashion (see Table [4.1] and Sect. 5 of this chapter).

\subsection{Vertex definition}

The vertex finding algorithm described in Sect. [3.12] was used to find the primary vertex of the event. In about $20 \%$ of the cases, a vertex was found in the $y$ view with no matching vertex in the $\mathrm{x}$ view; this was because the $\mathrm{x}$ view was at times affected by hardware problems. Such unmatched vertices were accepted; however, 
Table 4.1: Number of triggers

\begin{tabular}{|l|c|c|}
\hline Events & $\mathrm{Be}$ & $\mathrm{Al}$ \\
\hline Total number of triggers & 53953 & 40675 \\
\hline Events on DST & 14788 & 9249 \\
\hline Events with vertex & 13959 & 8127 \\
\hline
\end{tabular}

a visual inspection of the hits for about 25 such events showed that the vertex determination was, in fact, adequate.

The $z$ distribution for the found vertices is shown in Fig. [4.1]; the target segmentation is evident in the reproduced $z$ distribution; one can also see the last SSD beam station at $z \approx-20 \mathrm{~cm}$, the first downstream SSD station at $z \approx-6 \mathrm{~cm}$, and the second downstream SSD station at $z \approx 0 \mathrm{~cm}$; the other SSD stations are not mapped in the vertex distribution in the figure because, if an interaction occurs there, it does not produce enough hits in the SSD's to yield tracks.

Every event was required to have a vertex in the target region; the effects of that cut are summarized in Table [4.1] for $\mathrm{Al}$ and Be data. The fraction of events which have a vertex in the SSD is compatible with the interaction length of Si.

A sample of events for which no vertex was found was studied in some detail. Out of 30 events in which the vertex program found no vertex, a visual scan of the hits indicated that at most 2 may have had a vertex in the target. The other events were interactions in the magnet, interactions in the SSD's, or were events with no clear interactions.

\subsection{Pedestal and gain corrections}

The pulse height $P_{i}$ for each channel of the EMLAC is given by:

$$
P_{i}=A_{i}+G_{i} N_{i}
$$

where $N_{i}$ is the observed number of counts in the corresponding analog-to-digital converter (ADC), $A_{i}$ is the pedestal and $G_{i}$ is the gain for channel $i$. The constant $G_{i}$ 
Figure 1.1: z distribution of primary inleraction vertices for Be data

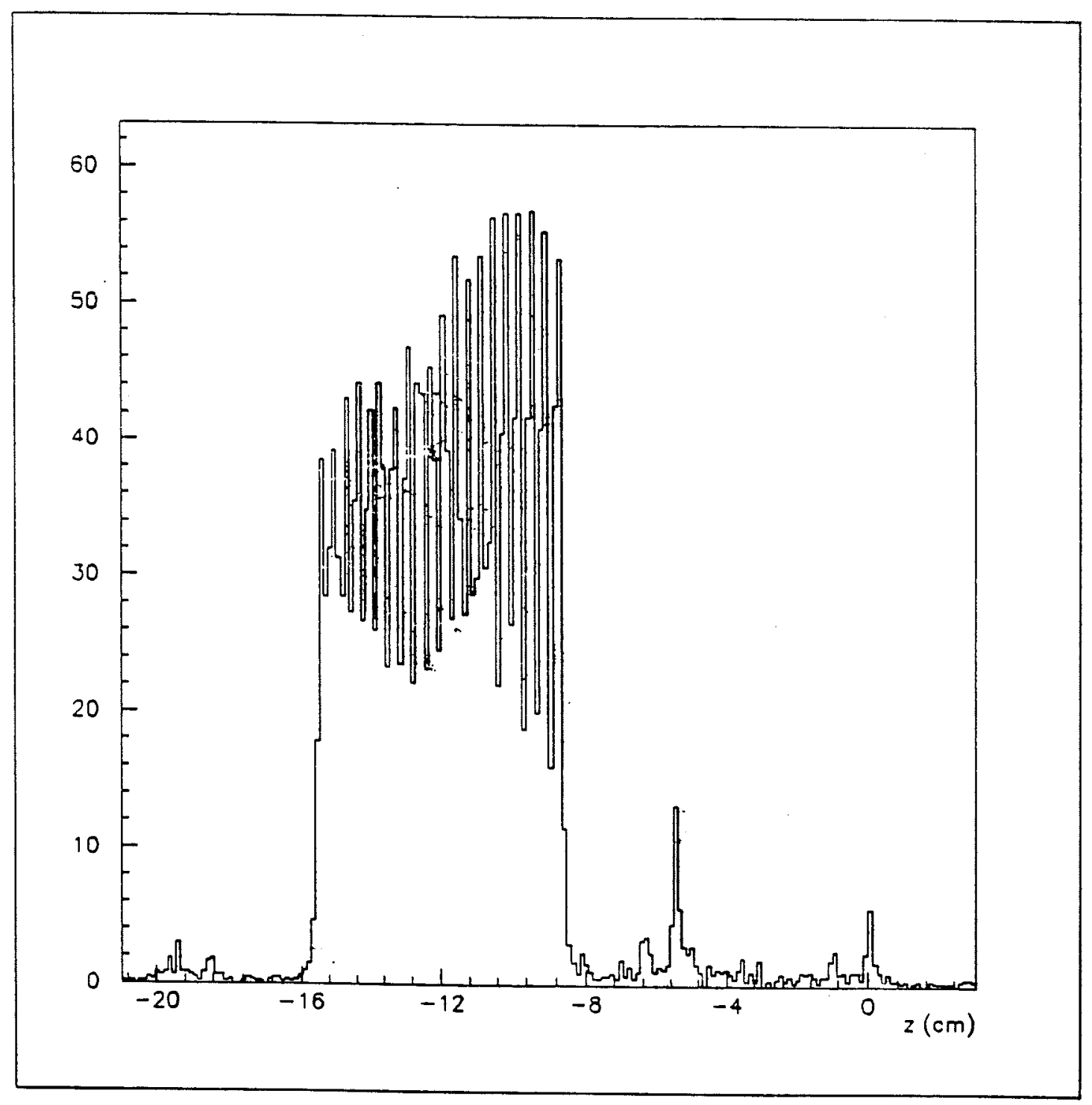


incorporates factors other than the electronics gains $E_{i}$ (e.g. inefficiency of charge collection, etc); at first we assumed the other factors to be constant over the entire detector i.e., $G_{i}=E_{i} k$. From a study of calibration runs using electron beams and from the mass value for high $p_{T} \pi^{0}$ 's, $k$ was initially set to $3.01 \mathrm{MeV} /$ count.

The pedestals and the electronic gains were determined every few hours using a calibration procedure that involved on-board calibrators in the amplifier cards; a data base was created to provide run-dependent corrections. The stability of these quantities was rather good. The electronic gains exhibited good uniformity, e.g., the standard deviation of the electronic gain distribution for all channels in the EMLAC was below $2.5 \%$.

The pedestals from the electronic calibration procedure were compared with pedestals determined with data where every channel of the EMLAC was read out (referred to as non zero-suppressed data). It was found that the pedestal value could be shifted typically by about 20 counts without a discernible reason or pattern; larger deviations occurred occasionally. Figure [4.2] shows the difference between the pedestal determination by the electronic calibration procedure and by an algorithm which fits the tail of the pulse height distribution to determine the pedestal for an empty channel. This difference is an indication of the error in the nominal pedestal value. Since there were not enough non zero-suppressed data to measure the pedestals, we had to rely on the electronics calibration procedure; this is a source of systematic error that will be mentioned in Chapt. 6 .

\subsection{Tail corrections for showers}

The zero suppression circuitry in the EWE (see Sect. [2.7]) was meant to eliminate channels below threshold. The nominal value of the zero suppression threshold $E_{t h}$ is given by:

$$
E_{t h}=50 m V(255 / T)(1 / 65)(1 / 2)(65535 \text { counts } / 2.5 V)=2570 \text { counts } / T
$$

where:

1. $50 \mathrm{mV}$ is the hardware window at the output of the test over threshold (TOT) circuitry in the EWE. 
Figure 4.2: Difrerence between the neminal perdestal value and the value determined from a fit to the tail of the pulse height distribution for the front section of the LAC, $\phi$ view, quadrant 3 for Rum 3055

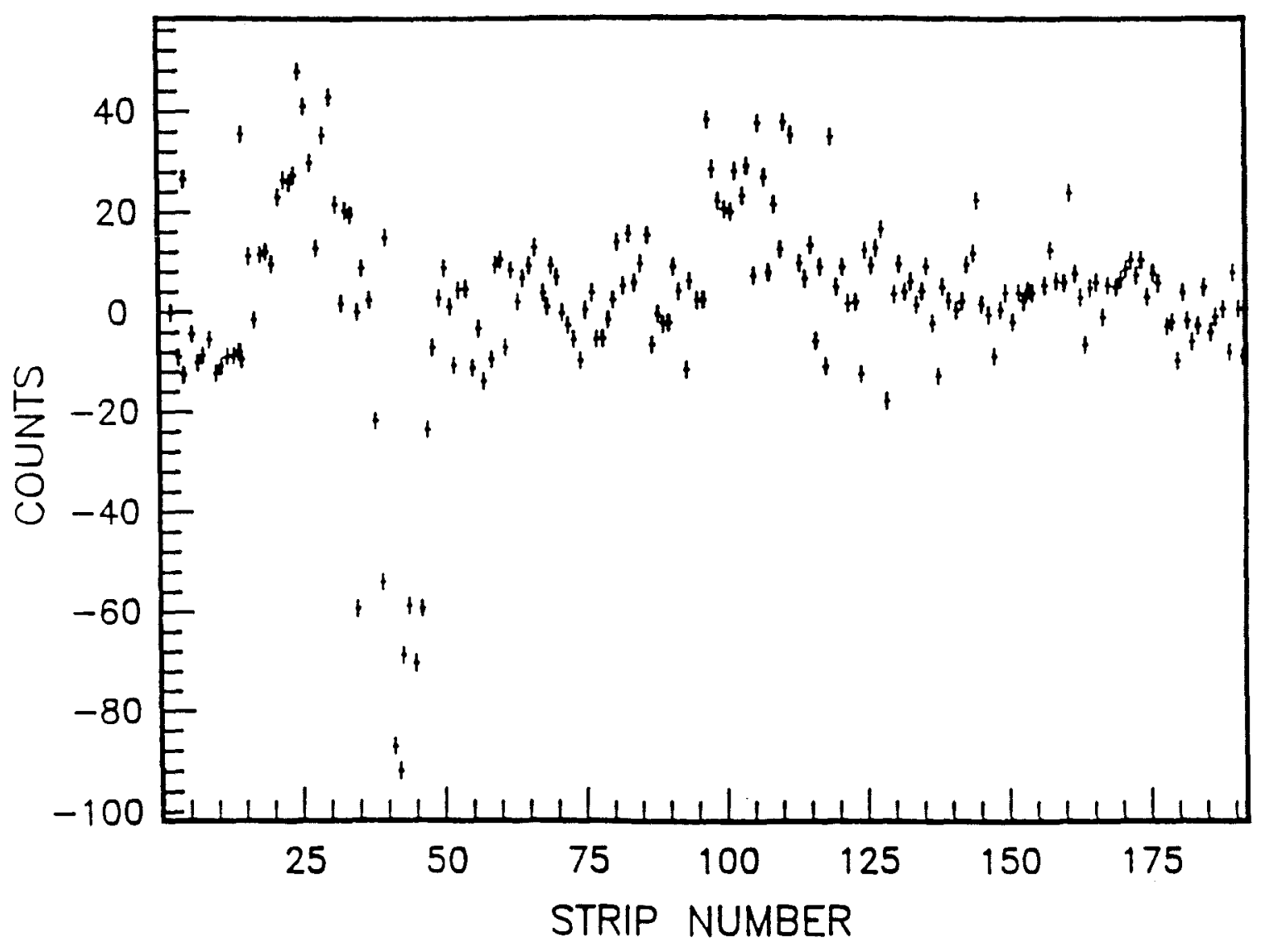


2. (T/255) is a scaling factor applied by the threshold digital-to-analog converter (DAC), where $T$ is the value of the decimal DAC setting for the run ( $T=61$ counts for the data studied here).

3. 65 is the hard-wired gain of the input to the TOT section of the EWE.

4. 2 is the hard-wired gain of the pedestal correction section of the EWE.

5. $2.5 \mathrm{~V} / 65535$ counts is the nominal gain of the ADC that we used.

The EWE circuitry suffered from stability problems that shifted the position of the zero-suppression window, causing more channels to be read out than desired. The largest event size that the on-line system could handle limited the value we could use for the threshold. For the runs studied here, the zero-suppression threshold was approximately $170 \mathrm{MeV}$.

The zero suppression threshold reduced the "tails" of the shower distribution, causing a loss of energy which was very significant for low energy showers. A correction (tail correction) to the shower energy was determined by calculating the integral of the shower shape for strips below threshold (Fig. [4.3]). The tail correction for isolated showers was typically $2.6 \mathrm{GeV}$; it increased very slowly with energy; it also varied as a function of the position in the EMLAC, mainly due to the variation in width of the $\phi$ strips with $\mathrm{r}$.

\subsection{Definition of the $\pi^{0}$ signal}

Showers in the EMLAC were required to be incident within a fiducial boundary in order to make sure that they were contained within the active volume. The centroids of reconstructed showers were required to be at least $3 \mathrm{~cm}$ away from the edge of the calorimeter.

Monte Carlo studies indicated that showers with energy less than about 3.5 $\mathrm{GeV}$ did not produce a signal in the EMLAC; therefore, any shower with less than $3.5 \mathrm{GeV}$ was rejected to protect against electronic noise and reconstruction errors.

Additional cuts were applied to reduce the hadronic background in the EMLAC; e.g., all showers with less than $1.5 \mathrm{GeV}$ in the front section of the EMLAC 
Figure 4.3: Calculated energy below threshold for a $5 \mathrm{GeV}$ shower at $r=52 \mathrm{~cm}$ versus threshold for zero suppression (the straight line is drawn to guide the eye)

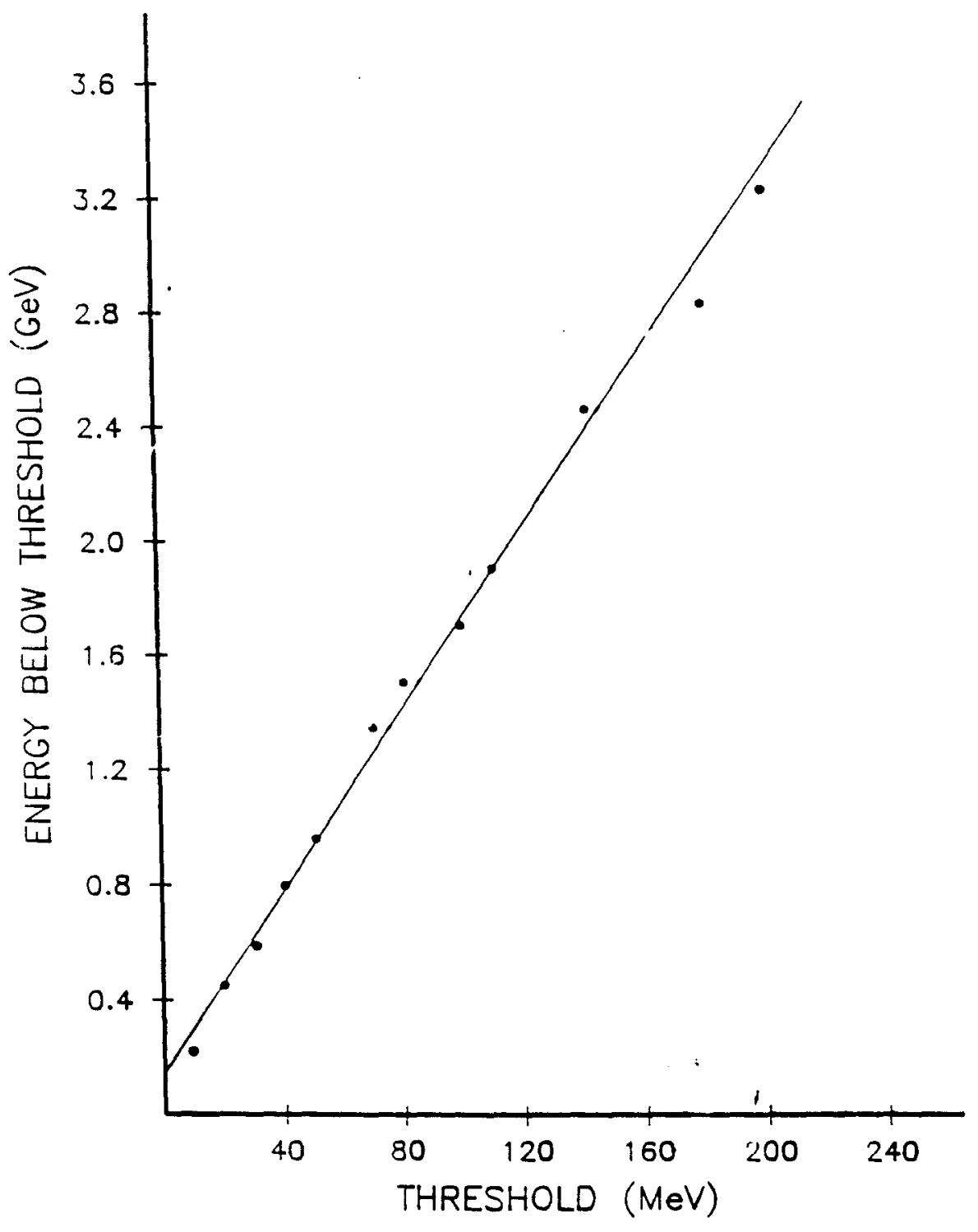


were discarded. Any shower with a charged track pointing to it within $2 \mathrm{~cm}$ was also rejected. The hadron calorimeter was not used for hadron rejection, since it did not have sufficient sensitivity for rejecting low-energy hadrons which contribute to our background.

The $\pi^{0}$ signal is identified in the $\gamma \gamma$ mode by calculating the invariant mass of all $\gamma \gamma$ pairs in each quadrant of the EMLAC; the branching ratio for this mode is $98.85 \pm 0.05 \%$. The relative angle between the photons is calculated from the positions of the photons and the distance of the EMLAC from the target; since the lifetime of a $\pi^{0}$ is $10^{-16} \mathrm{sec}$, even the most relativistic ones decay within the target.

We fitted the mass distribution (including all $\gamma \gamma$ combinations in each quadrant) with a Gaussian term which represents the $\pi^{0}$ peak and a simple term to describe the background and wrong combinations:

$$
A e^{\frac{-\left(m-m_{0}\right)^{2}}{2 \sigma^{2}}}+C m^{\alpha} e^{-m \beta}
$$

The mean of the Gaussian distribution $m_{0}$ is the fitted $\pi^{0}$ mass and the standard deviation $\sigma$ measures the width or resolution of the $\pi^{0}$ mass peak. The $\pi^{0}$ mass distribution was also studied separately in each quadrant (see Table [4.2]), which combines the $\mathrm{Al}$ data with the $\mathrm{Be}$ data). It was also noticed that the value of the $\pi^{0}$ mass peak decreased as a function of the radial distance from the center of the detector; the largest variation (almost $5 \%$ ) occured in the inner region of the detector. Since this behaviour was not observed in the Monte Carlo models, it was attributed to non-uniformity in the detector rather than to the reconstruction program. The energy scale was first adjusted so that the position of the peak in each quadrant was at the right value for the $\pi^{0}$ mass (quadrant correction), then another correction was applied to eliminate the r-dependence of the $\pi^{0}$ mass. Although the reasons for the quadrant dependence or the $r$-dependence of the $\pi^{0}$ mass is not yet fully understood, we believe that the our correction procedure improves the uniformity of detector response. The results of the fits after the energy rescaling are given in table [4.3]; the $\chi^{2}$ refers to the fit to the invariant mass distribution, which includes both the terms for the $\pi^{0}$ and the background. The corrections described above improved the width of the observed $\pi^{0}$ signal by about $2 \mathrm{M} \mathrm{eV} / \mathrm{c}^{2}$.

A $\pi^{0}$ was defined as a photon pair with a reconstructed mass in the range (135 \pm 45$) ~ M e V / c^{2}$. In order to extract the $\pi^{0}$ signal, we had to substract the effect of the background under the $\pi^{0}$ peak. We defined sidebands between $50-90 \mathrm{MeV} / \mathrm{c}^{2}$ and $190-230 \mathrm{MeV} / \mathrm{c}^{2}$. The number of events in the sidebands was weighted so that 
Table 4.2: Dependence of the $\pi^{0}$ mass on the EMLAC quadrant

\begin{tabular}{|l|c|c|}
\hline Quadrant & $m_{0}\left(\mathrm{MeV} / \mathrm{c}^{2}\right)$ & $\sigma\left(\mathrm{MeV} / \mathrm{c}^{2}\right)$ \\
\hline Quadrant 1 & $139 \pm 0.8$ & $14.1 \pm 0.8$ \\
\hline Quadrant 2 & $136 \pm 0.7$ & $14.2 \pm 0.6$ \\
\hline Quadrant 3 & $150 \pm 1.0$ & $17.7 \pm 1.0$ \\
\hline Quadrant 4 & $140 \pm 0.7$ & $15.7 \pm 0.7$ \\
\hline
\end{tabular}

Table 4.3: Fitted values for the $\pi^{0}$ mass and width

\begin{tabular}{|l|c|c|}
\hline Parameter & $\mathrm{Be}$ & $\mathrm{Al}$ \\
\hline$A$ & $361 \pm 16$ & $196 \pm 12$ \\
\hline$m_{0}\left(\mathrm{MeV} / \mathrm{c}^{2}\right)$ & $134.9 \pm 0.6$ & $133.6 \pm 0.7$ \\
\hline$\sigma\left(\mathrm{MeV} / \mathrm{c}^{2}\right)$ & $14.1 \pm 0.5$ & $12.4 \pm 0.7$ \\
\hline$\chi^{2} / 44$ degrees of freedom & 38.3 & 34.0 \\
\hline
\end{tabular}


the weighted number was equal to the integral of the background under the $\pi^{0}$.

The histograms for Be data in Fig. [4.4] and for Al data in Fig. [4.5] show the invariant mass distributions with the fit to the $\pi^{0}$ signal and background. As discussed earlier in this section, the showers had to satisfy the following requirements to be entered in the invariant mass distribution histogram:

1. The total shower energy had to be larger than $3.5 \mathrm{GeV}$;

2. The energy deposited in the front section of the calorimeter had to be larger than $1.5 \mathrm{GeV}$;

3. The vertex for the corresponding event had to be within the target region;

4. No charged tracks had to point to the shower within $2 \mathrm{~cm}$.

After the fitting procedure was established, it was also used to study the effects of any of the cuts on the yields. A separate fit to the $\gamma \gamma$ invariant mass distribution was done after the application of each cut.

The cumulative effects of the cuts are shown in Table [4.4] for Be data and in Table [4.5] for Al data; each row indicates the effects of the relative cut applied in conjunction with the previous cuts. The column labeled 'Background' gives the integral in the $\pi^{0}$ mass region of the function representing the best fit to the background. The column labeled ' $\pi^{0}$ candidates' is the signal, namely the difference between the number of entries in the $\pi^{0}$ region and the integral of the background function in that region. The number of ' $\pi^{0}$ candidates' is affected by the statistical fluctuactions of the background. The requirement that the event must have a vertex in the target eliminates any $\pi^{0}$ 's produced in interactions occurring elsewhere; the loss is larger for the Al target because that target has a smaller interaction length than the Be target (see Table [2.1]). The cuts appear to improve the signal over background ratio with only a small loss in signal.

\subsection{Background subtraction}

We expected that the characteristics of the added events from the sidebands were similar to those of the background events in the $\pi^{0}$ region; for instance, the asymme- 
Figure 4.f: $\gamma \gamma$ invariant mass distribution for Br data with the fit. (solid line) to the $\pi^{0}$ signal and background

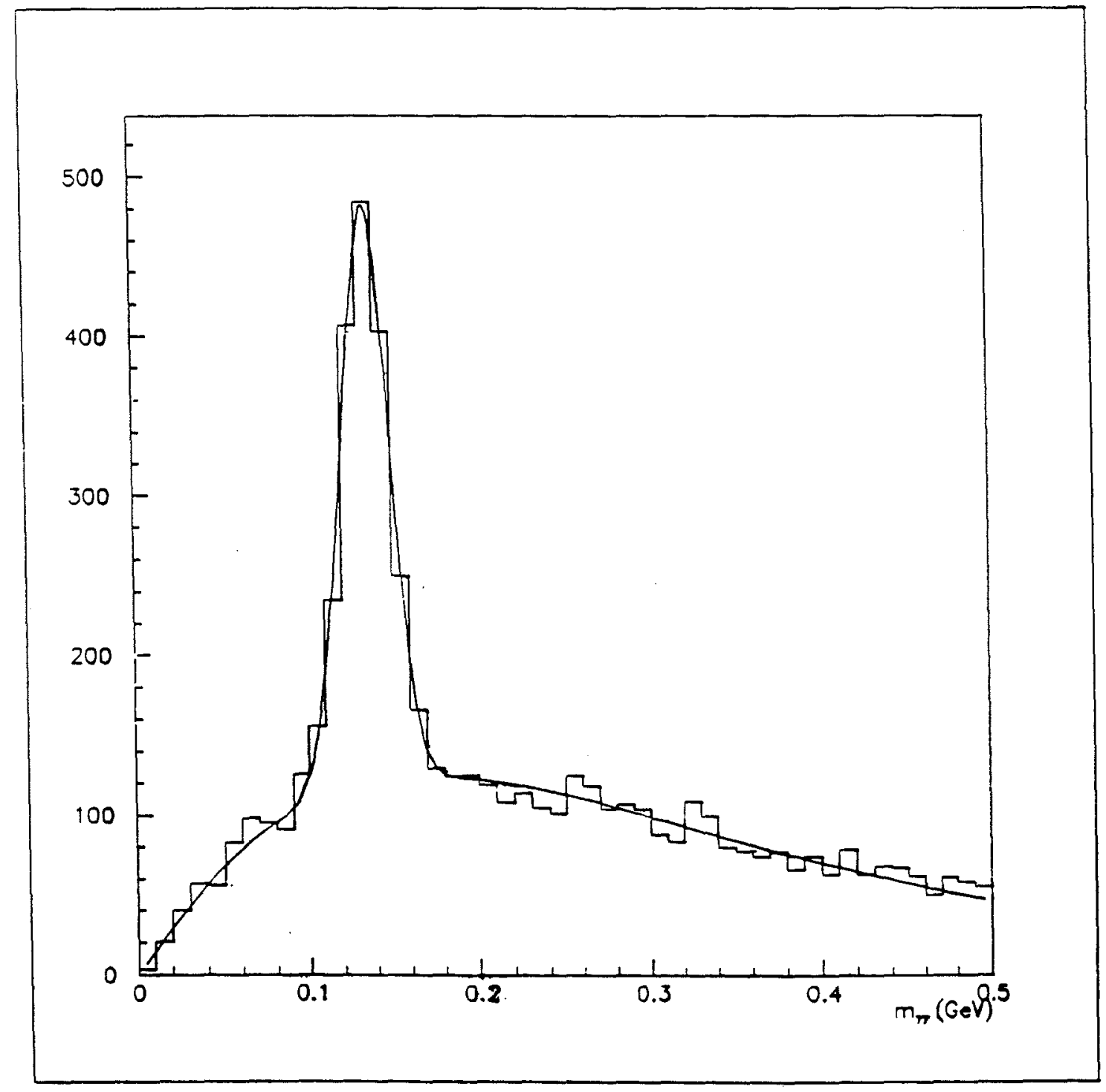


Figure 4.5: $\gamma \gamma$ invariant mass distribution for . 11 dala with the fit (solid line) to the $\pi^{0}$ signal and background

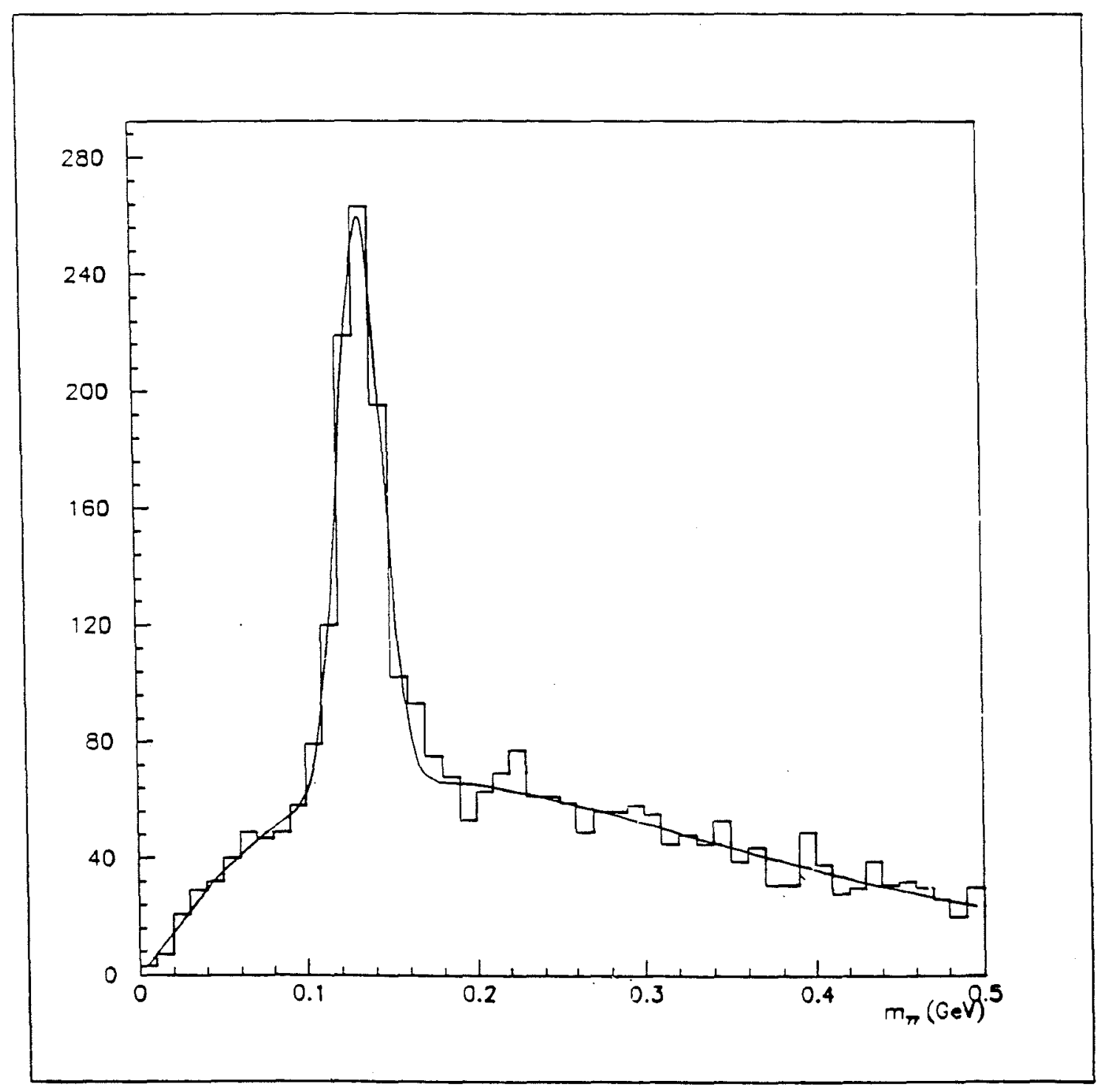


Table 4.4: Cuts for Al data

\begin{tabular}{|l|c|c|}
\hline Cuts & $\pi^{0}$ candidates & Background \\
\hline Energy cut $\left(E_{\gamma}>3.5 \mathrm{GeV}\right)$ & 814 & 1693 \\
\hline + Front energy cut $\left(E_{\gamma, f}>1.5 \mathrm{GeV}\right)$ & 816 & 1085 \\
\hline + Vertex in the target & 573 & 779 \\
\hline + Track cut & 586 & 589 \\
\hline
\end{tabular}

Table 4.5: Cuts for Be data

\begin{tabular}{|l|c|c|}
\hline Cuts & $\pi^{0}$ candidates & Background \\
\hline Energy cut $\left(E_{\gamma}>3.5 \mathrm{GeV}\right)$ & 1615 & 2484 \\
\hline + Front energy cut $\left(E_{\gamma, f}>1.5 \mathrm{GeV}\right)$ & 1495 & 1704 \\
\hline + Vertex in the target & 1287 & 1504 \\
\hline + Track cut & 1312 & 1090 \\
\hline
\end{tabular}

try distribution (for a definition of asymmetry, see Sect. [3.10]) in the left sideband is steeper than the asymmetry distribution in the peak region; however, the asymmetry distribution in the right sideband is less steep than in the peak region, so that it is not unreasonable to assume that the combined asymmetry distribution of the sidebands should provide a good description of the background under the $\pi^{0}$ peak.

In order to calculate any quantity of scientific interest, we used the following procedure:

- the events in the peak region and in the sidebands were weighted by the Monte Carlo correction (see Chapt. 5) which is a function of $p_{T}$ and rapidity;

- the distribution for the quantity of interests was evaluated separately in the peak and sideband regions;

- the sideband distribution was then subtracted from the peak distribution. 


\subsection{Determination of the incident beam}

In order to normalize the cross section, we needed to determine the live triggerable beam, which is the total incident beam for intervals in which we took data, i. e. when the experiment was "live". The live triggerable beam was calculated using the following formula:

"live triggerable beam" = LIVE_BM.BHB $C_{1} \bullet C_{2} \bullet C_{3}$

where LIVE_BM.BHB is the number of beam particles during the time the computer could take data, $C_{1}$ is a correction for interactions too close in time to the one of interest, $C_{2}$ is a correction for trigger dead time (computer-live but trigger-dead) and $C_{3}$ is a correction for the effects of the SCRKILL and veto wall (see Sect. [2.8]). The expressions for the above corrections are:

\section{1. $C_{1}=$ LIVE_CLINT/LIVE_INT}

2. $C_{2}=($ PRETRIGGER_OR+NO_PRETRIGGER)/LIVE_INT1

3. $C_{3}=\left[1-G A T E D_{-} I N T 1\left(S C R K I L L+V E T O_{-} W A L L\right)\right] / G A T E D_{-} I N T 1$

The definition for all these quantities was given in Sect. [2.8].

The livetime is determined as:

LIVETIME="live triggerable beam"/BEAM.BHB

The livetime varied between $69 \%$ and $31 \%$ in different runs. $C_{1}$ was typically $0.995, C_{2}$ was typically 0.99 ; therefore these corrections were negligible; $C_{3}$ was typically 0.83 . The typical value of the livetime corresponded to a readout time of $17 \mathrm{~ms} /$ event, which is compatible with the values measured using the computer busy signals.

The interaction definition (see Sect. [2.8]), which required at least one interaction counter to fire, was very liberal. If at least two counters were required, then about half of the events were rejected; if two counters were required for events with at least two showers in a quadrant of the EMLAC, then only $\approx 2.5 \%$ of such events were rejected. The two counter requirement also improved the uniformity between counting rates in different counters, as shown in Table [4.6]. The interaction rate measured by taking the ratio LIVE_INT1/LIVE_BEAM was about twice the expected rate; by requiring at least two counters to fire, the interaction rate became compatible with the value expected from the interaction cross section. 
Table 4.6: Interaction counters for Run 2375 (Al target)

\begin{tabular}{|l|c|c|c|c|}
\hline Counter & SE1 & SW1 & SE2 & SW2 \\
\hline number of hits & 326830 & 210080 & 379390 & 478620 \\
\hline $\begin{array}{l}\text { number of hits } \\
\text { in events with 2 counters }\end{array}$ & 28400 & 23880 & 28170 & 28100 \\
\hline
\end{tabular}

The interaction rates, dead times and total amount of beam were studied in detail in each run presented here; counting rates were in agreement between different runs; the scalers showed good stability for varying beam intensities. Significant problems were not expected, since the system was designed to perform reliably at far larger interaction rates (up to $1 \mathrm{MHz}$; the typical interaction rate in these data is $\approx 1 \mathrm{~Hz}$ ).

Prescaled interaction triggers were also included in the standard run. The interaction rate was determined in a typical run and found to be compatible within errors with the interaction rate observed in the dedicated interaction triggers. It was not possible to carry out this test to better than about $10 \%$ accuracy because there was no standard run containing prescaled interactions with exactly the same target as used in the dedicated interaction runs. 


\section{Chapter 5}

\section{MONTE CARLO CORRECTIONS}

\subsection{General organization}

In order to measure inclusive cross sections it is essential to take into account the geometric acceptance, the reconstruction efficiency and the effects of the cuts, by applying appropriate corrections to the data. These corrections have been calculated using the E706 GEANT Monte Carlo program (see Sect. [3.2]).

\section{$5.2 \quad \pi^{0}$ generation and decay}

$\pi^{0}$ 's were assumed to be produced in the target; for any given $\pi^{0}$ the four-momenta of the two decay photons were then calculated. Because $\pi^{0}$ 's are spin zero particles, they decay isotropically in their rest frame; the photon four-momenta obtained in the $\pi^{0}$ rest frame were boosted to the laboratory frame and the positions of the two photons at the EMLAC then calculated. The corresponding showers were generated using tabulated lateral and longitudinal shower profiles, as described in Chapt. 2. These shower profiles were determined using a full GEANT simulation of the shower development.

From a study of the characteristics of interaction triggers, we found that the average number of peaks in a "group" (Table [5.1]) is very close to one and the typ- 
Table 5.1: Number of peaks per "group" for Run 2379 (Be)

\begin{tabular}{|l|c|}
\hline number of "groups" with I peak & 19742 \\
\hline number of "groups" with 2 peaks & 1129 \\
\hline number of "groups" with 3 peaks & 79 \\
\hline number of "groups" with 4 peaks & 12 \\
\hline number of "groups" with more than 4 peaks & 0 \\
\hline
\end{tabular}

ical shower multiplicity in each correlation region (see Sect. [2.4] for the definitions of "group" and correlation region) does not exceed two showers. This relatively low multiplicity may be explained by the energy cuts applied to the showers; namely, since the number of photons increases with decreasing energy cut-off, an energy cut has the effect of reducing the multiplicity. Consequently, Monte Carlo events with only one $\pi^{0}$ provide an adequate model for the low energy events studied in this thesis.

\subsection{Calculation of the Monte Carlo corrections}

The phase space was divided into $18 p_{T}$ bins of the same size, from 0.6 to $3.6 \mathrm{GeV} / \mathrm{c}$ and into 12 rapidity $(y)$ bins of equal size, from -1 to 1 (for a definition of these quantities, see Chapt. 1). In this chapter we always calculate the rapidity in the center of mass frame; the corresponding value of the rapidity in the laboratory frame can be obtained by adding 3.52 to that of the center of mass rapidity.

We generated $1500 \pi^{0}$ events in each $\left(y, p_{T}\right)$ cell; the events were then reconstructed and subjected to the same cuts that we applied to real data. The ratio of the number of reconstructed $\pi^{0}$ 's to the number of generated $\pi^{0}$ 's provided the Monte Carlo weight correcting each $\left(y, p_{T}\right)$ cell (Fig. [5.2]); it includes the geometrical acceptance, the reconstruction efficiency and the effects of the analysis cuts. The Monte Carlo correction table was determined by taking the inverse of the weights; the correction for each event was then calculated through a linear interpolation between the values in the acceptance table. 
Figure 5.1: Monte Carlo weights for $\pi^{3}$ production as a function of $p_{r}$ and center-of-mass rapidity y for cerents from the interaction trigger. The absolute scale is indicaled by the weight of 1 in the lower right corner.

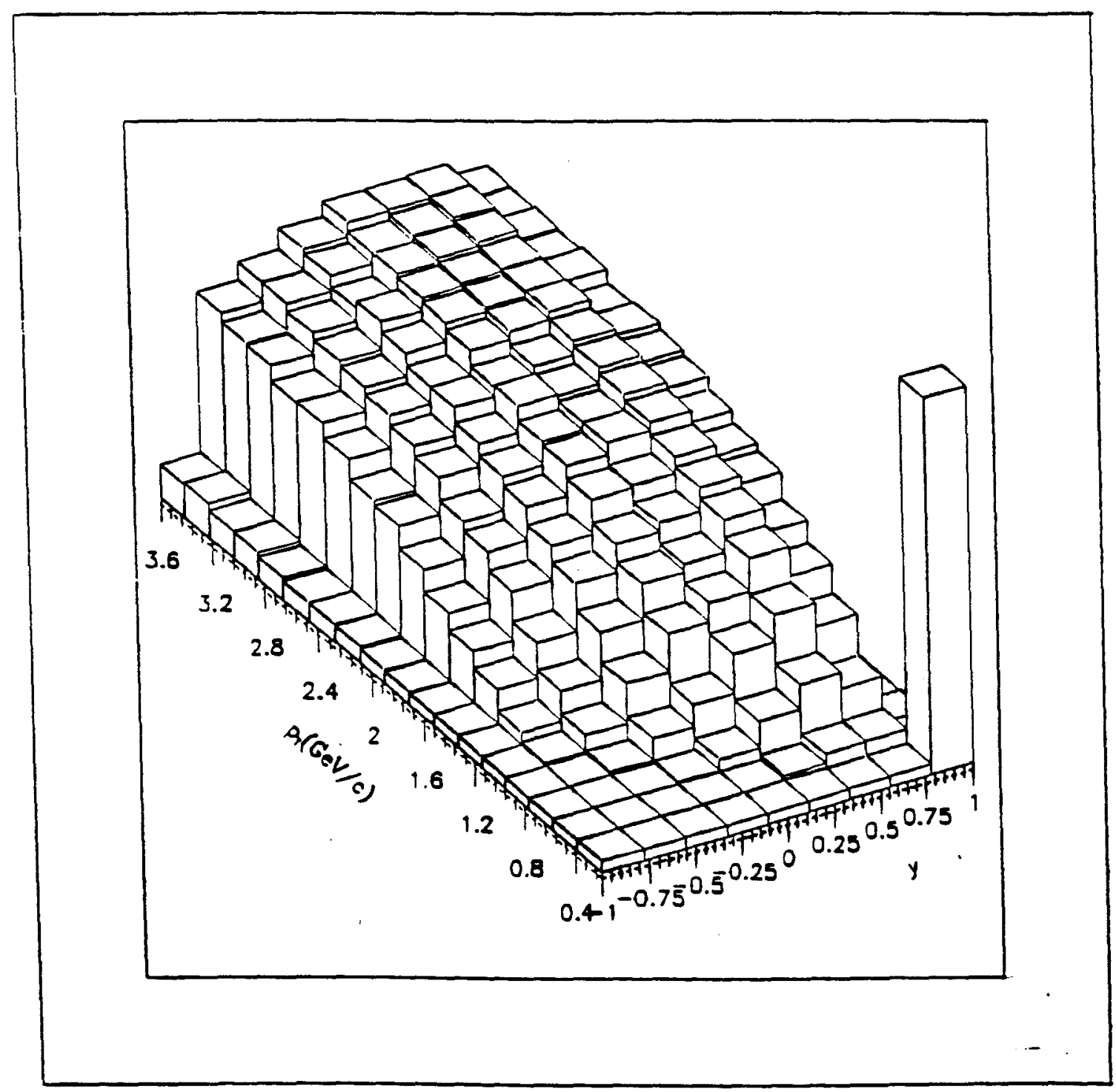




\subsection{Comparison of the Monte Carlo model with the data}

It is essential to make sure that the Monte Carlo package used to determine the Monte Carlo corrections is able reproduce the relevant characteristics of the data.

We generated a sample of $33,000 \pi^{0}$ 's according to the observed $p_{T}$ and $y$ distributions in the real data. We could not produce a double differential distribution in $p_{T}$ and $y$ from the real data because of the limitations in our statistics. The distribution however was sampled using the following procedure:

1. The four-momentum of each $\gamma \gamma$ combination in the $\pi^{0}$ region was determined.

2. The Monte Carlo correction for the corresponding y and $p_{T}$ was calculated.

3. $\mathrm{N} \pi^{0}$ 's with the same $y, p_{T}$ and with a uniform $\phi$ distribution were generated. $\mathrm{N}$ was chosen to be proportional to the Monte Carlo correction for the corresponding $\gamma \gamma$ combination.

The generated events were reconstructed and analysed using the same cuts as applied to the real data. Since the statistics for each target are limited, for this study data from all targets were combined. We compared the asymmetry distribution (for a definition of asymmetry, see Sect. [3.10]) for these Monte Carlo events with the asymmetry distribution of the $\pi^{0}$ 's in the real data and we found them to agree well within errors (Fig. [5.3]); this result does not appear to be sensitive to small changes in the fit to the background.

Because the $\pi^{0}$ is a spin 0 particle, the event distribution in asymmetry should in principle be independent of asymmetry. The reason the measured distribution decreases rapidly with increasing asymmetry is that the typical $\pi^{0}$ energy is not much larger than the energy $E_{\min }$ below which showers are not reconstructed $\left(E_{\min } \approx 3.5\right.$ $\mathrm{GeV})$. For any given $\pi^{0}$ energy $E$ the asymmetry $A(E)$ is constant up to the maximum asymmetry $A_{\max }=1-2 E_{\min } / E$. The experimental asymmetry distribution is, of course, the convolution of the distributions $A(E)$ with the rapidly falling $\pi^{0}$ energy spectrum, and consequently drops rapidly with increasing asymmetry.

We also compared the $\gamma \gamma$ mass distribution obtained using the Monte Carlo (Fig [5.4]) with the same distribution from the Be data. The position of the peak, 
Figure 5.2: Asymmetry distribution for $\pi^{\prime \prime}$ production from the Monte Carlo predietion for Be data; the circles represent. the Jlonte ('arlo prediction (to eliminate statistical fluctuctions in the Monte ('arlo curre. a smoothing algorithm was applied)

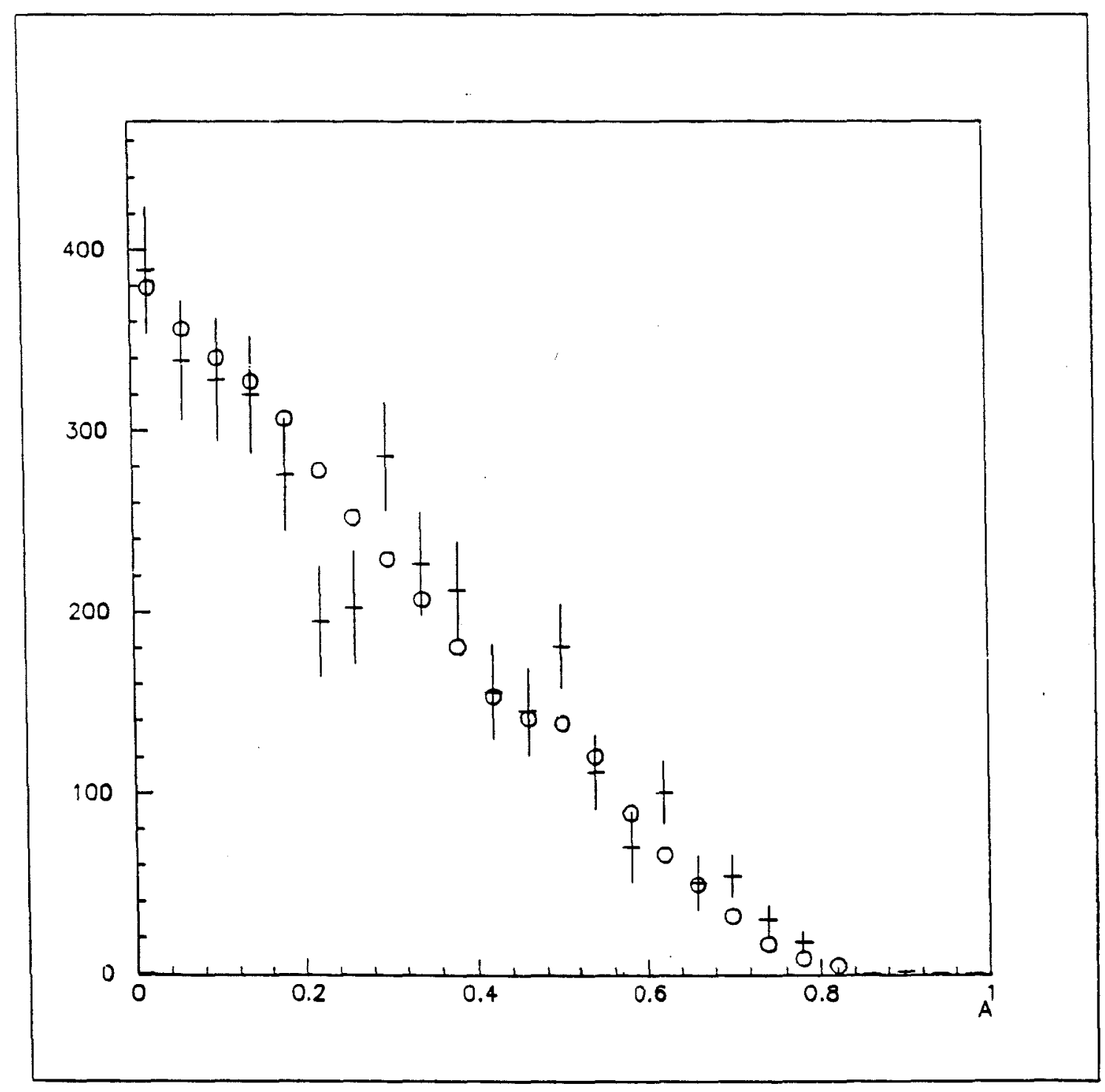


which corresponds to the $\pi^{0}$ mass, agrees in the two plots. On the other hand the width of the Monte Carlo distribution is considerably narrower $\left(\sigma \approx 11 \mathrm{M} \mathrm{eV} / \mathrm{c}^{2}\right.$ for the Monte Carlo, $\sigma \approx 14 \mathrm{MIeV} / \mathrm{c}^{2}$ for the data). This discrepancy can be explained by the fact that the Monte Carlo does not include all sources of uncertainty. In particular, the errors in the pedestal determination and in the centering of the zero suppression window could not be included in the Monte Carlo, since those errors do not as yet appear to follow a discernible pattern. This subject will be discussed again in the next chapter, because we believe this is one of the largest sources of systematic error in our measurement. 


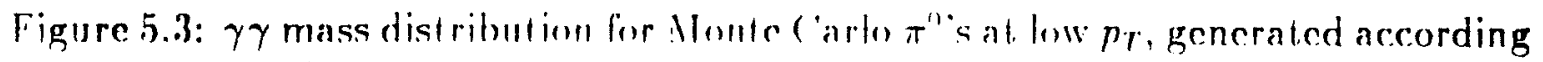
to the spectra observed in the real data.

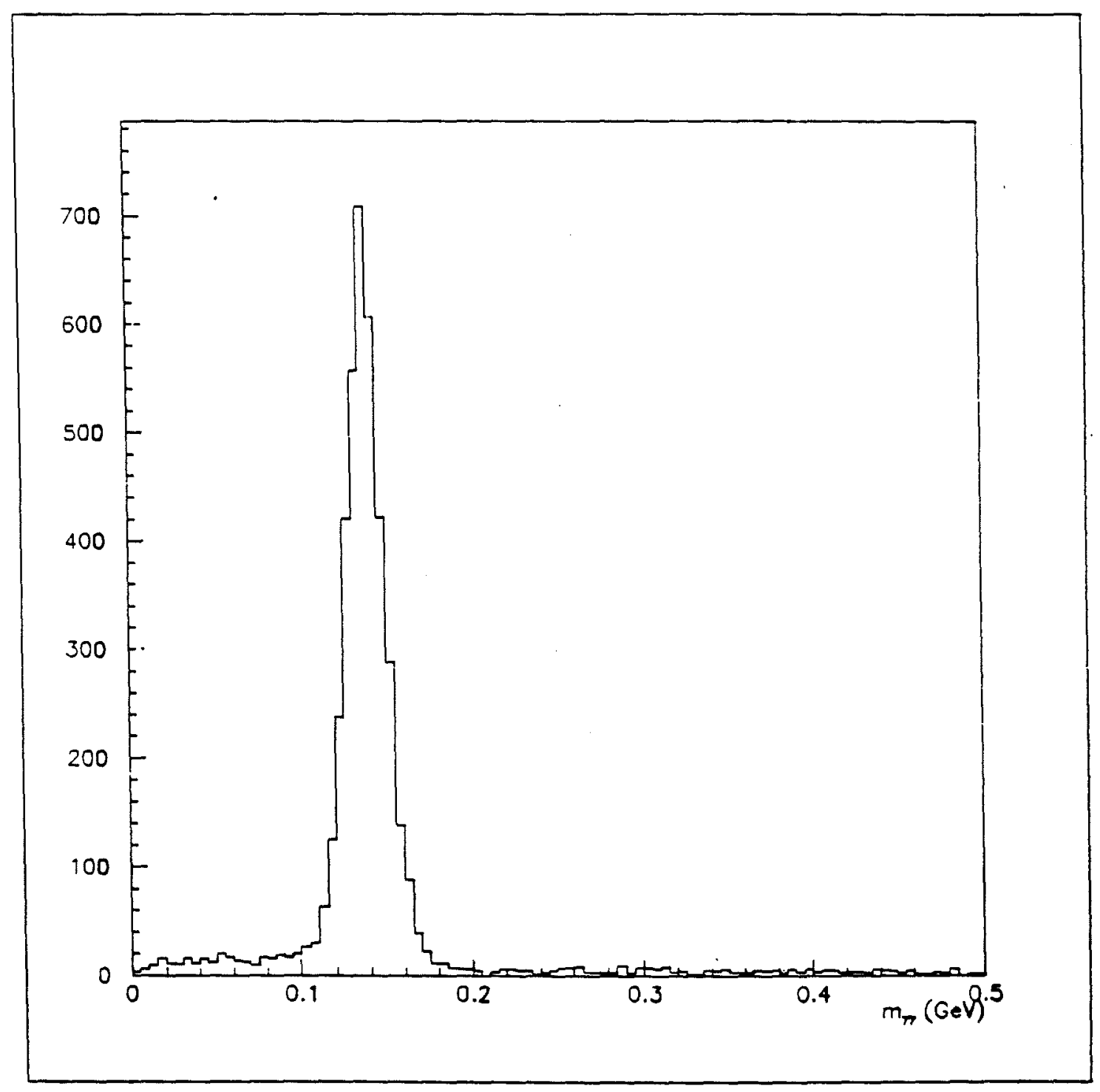




\section{Chapter 6}

\section{RESULTS AND CONCLUSIONS}

\subsection{General considerations}

In this chapter the results of this work are presented. Section 2 deals with the normalization of the inclusive cross section, Section 3 presents our results for the cross sections, Section 4 deals with the atomic dependence of the cross sections, Section 5 provides a discussion of the sources of systematic error in our measurements and Section 6 presents the implications of our results and our conclusions.

\subsection{Cross section normalization}

The invariant cross section per nucleus can be written as

$$
E \frac{d \sigma}{d^{3} p}=\frac{d \sigma}{d y p_{T} d p_{T} d \phi}=\frac{A}{\rho l N_{0}} \frac{Y}{F} \frac{\left(1+l / 2 \lambda_{a b_{0}}\right)}{\Delta y \Delta \phi p_{T} \Delta p_{T}}
$$

where $A, \rho, \lambda_{a b}$ and $l$ are the atomic weight, density, absorbtion length and length of the target respectively, (the values for these quantities were given in Table [2.1]); $N_{0}$ is Avogadro's number, $\mathrm{Y}$ is the yield of events multiplied by the Monte Carlo correction for acceptance (see Chapt. 5), $\mathrm{F}$ is the total number of beam particles, and $\Delta y \Delta \phi_{p_{T}} \Delta p_{T}$ is a volume element in phase space. We included a factor which 
is a thin target correction for absorption of the incoming beam inside the target and assumes that interactions occur on average in the middle of the target. The yield of events was corrected for the fraction of photons which converted into $e^{+} e^{-}$ pairs in the target, as calculated from the target radiation length. The correction for the branching ratio was included in the Monte Carlo correction.

\subsection{Inclusive cross sections for $\pi^{0}$ production}

The differential cross section per nucleus for $\pi^{0}$ production (averaged over azimuth) presented in this section can be written as:

$$
\frac{1}{\pi \Delta y} \frac{d \sigma}{d p_{T}^{2}}=\frac{1}{\pi \Delta y} \int_{y_{1}}^{y_{2}} \frac{d \sigma\left(y, p_{T}\right)}{d p_{T}^{2} d y} d y
$$

where $y_{1}$ and $y_{2}$ are the limits of the rapidity interval taken into consideration and $\Delta_{y}=y_{2}-y_{1}$. The quantity defined in Eq. (1) is displayed in Tables 6.1 and 6.2 and Figs. 6.1-6.4, 6.7 and 6.8.

The cross section was measured as a function of $p_{T}$ in the two following center of mass rapidity $\left(y_{c m}\right)$ intervals for both Be and Al targets:

1. central region (defined by $-0.25<y_{c m}<0.25$ ); the corresponding results are shown in Table [6.1], and in Fig. [6.1] and Fig. [6.3] for the Be and Al target respectively;

2. forward region (defined by $0.25<y_{c m}<0.75$ ); the corresponding results are shown in Table [6.2], and in Fig. [6.2] and Fig. [6.4] for the Be and Al target respectively. We were able to extend our measurement to lower $p_{T}$ in the forward region because we had better acceptance there (see Fig. [5.1]).

The errors shown in the figures are given by the square root of the sums of the squares of the Monte Carlo corrections (see Chapt. 5) in each $p_{T}$ bin; since the Monte Carlo correction was parametrized as a function of $p_{T}$ and $y_{\mathrm{cm}}$, the errors in each $p_{T}$ bin include contributions due to the variations of the weights as a function of rapidity.

The implications of our measurement of the cross sections described above will be given in Sect. [6.6], where the results of fits to the cross sections are also discussed and values are given for the integrated cross sections. 
Table 6.1: $\frac{1}{\pi \Delta y} \frac{d \sigma}{d p_{T}^{2}}\left(m b /(G e V / c)^{2}\right)$ (per nucleus) for $\pi^{0}$ production in collisions at $530 \mathrm{GeV},-0.25<y_{\mathrm{cm}}<0.25$

\begin{tabular}{|l|c|c|}
\hline$p_{T}(\mathrm{GeV} / \mathrm{c})$ & $\pi^{-} \mathrm{Be}$ & $\pi^{-} \mathrm{Al}$ \\
\hline $0.7-0.9$ & $12.3 \pm 2.6$ & $24.0 \pm 13.3$ \\
\hline $0.9-1.1$ & $3.54 \pm 0.57$ & $10.7 \pm 2.4$ \\
\hline $1.1-1.3$ & $1.46 \pm 0.22$ & $1.40 \pm 0.92$ \\
\hline $1.3-1.5$ & $0.36 \pm 0.10$ & $0.90 \pm 0.35$ \\
\hline $1.5-1.8$ & $0.145 \pm 0.044$ & $0.44 \pm 0.16$ \\
\hline $1.8-2.1$ & $0.057 \pm 0.019$ & $0.271 \pm 0.090$ \\
\hline
\end{tabular}

Table 6.2: $\frac{1}{\pi \Delta y} \frac{d \sigma}{d p_{T}^{2}}\left(\mathrm{mb} /(\mathrm{GeV} / \mathrm{c})^{2}\right)$ (per nucleus) for $\pi^{0}$ production in collisions at $530 \mathrm{GeV}, 0.25<y_{\mathrm{cm}}<0.75$

\begin{tabular}{|l|c|c|}
\hline$p_{T}(\mathrm{GeV} / \mathrm{c})$ & $\pi^{-} \mathrm{Be}$ & $\pi^{-} \mathrm{Al}$ \\
\hline $0.6-0.7$ & $22.2 \pm 3.3$ & $54.2 \pm 12.5$ \\
\hline $0.7-0.8$ & $13.4 \pm 1.9$ & $20.3 \pm 7.4$ \\
\hline $0.8-0.9$ & $4.40 \pm 1.0$ & $24.0 \pm 4.9$ \\
\hline $0.9-1.0$ & $4.29 \pm 0.72$ & $8.7 \pm 2.9$ \\
\hline $1.0-1.2$ & $1.29 \pm 0.26$ & $5.1 \pm 1.1$ \\
\hline $1.2-1.4$ & $0.52 \pm 0.13$ & $2.67 \pm 0.58$ \\
\hline $1.4-1.8$ & $0.232 \pm 0.038$ & $0.67 \pm 0.16$ \\
\hline $1.8-2.2$ & $0.067 \pm 0.018$ & $0.137 \pm 0.061$ \\
\hline
\end{tabular}


Figure 6.1: $\frac{1}{\pi \Delta y} \frac{d \sigma}{d r_{T}^{2}}\left(\mathrm{mb} /(G \mathrm{cl} / \mathrm{c})^{2}\right)$ (per nucleus) for $\pi^{0}$ production in $\pi^{-}$Be collisions at $530 \mathrm{GeV},-0.25<y_{\mathrm{cm}}<0.25$

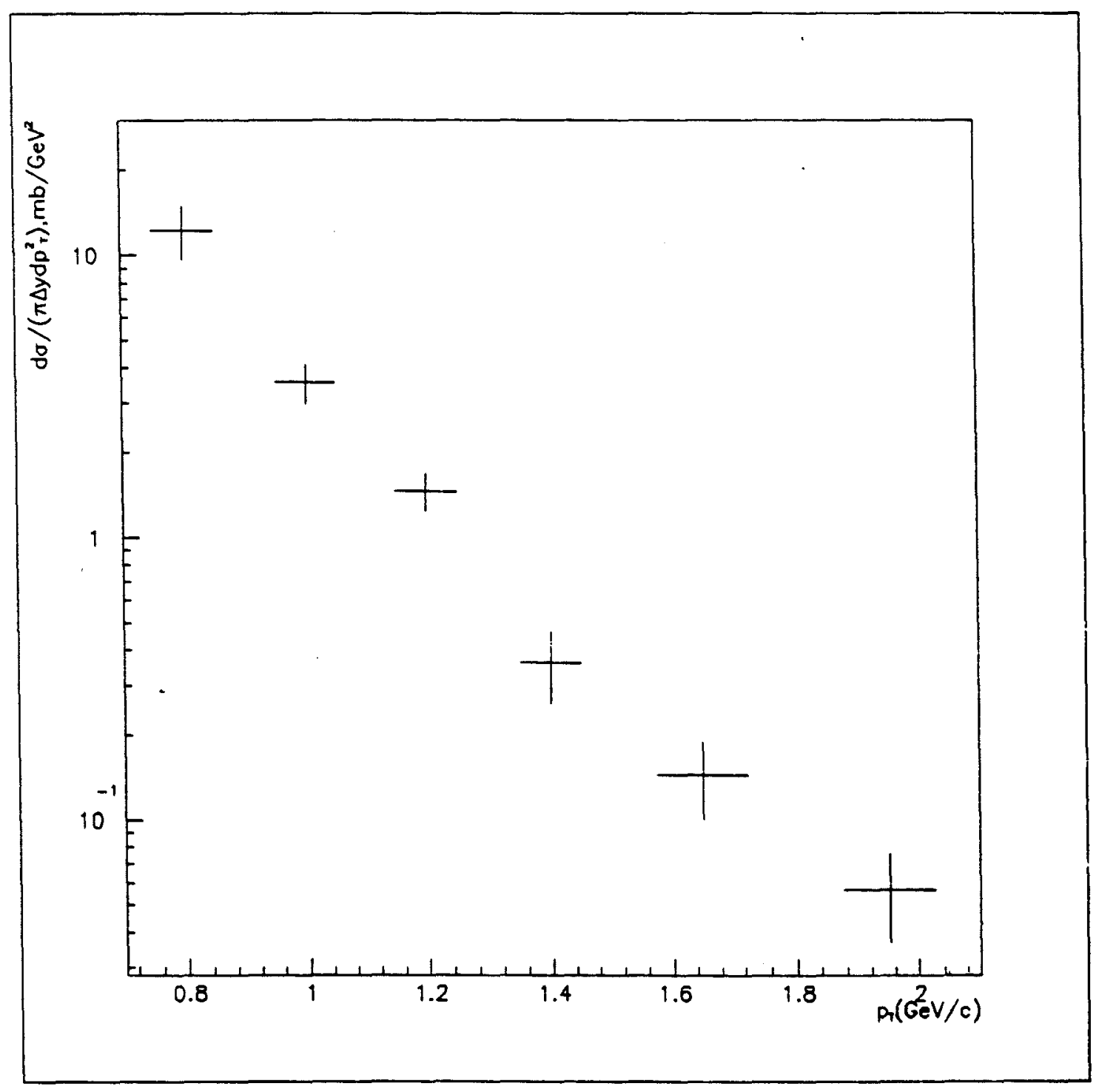


Figure 6.2: $\frac{1}{\pi \Delta y} \frac{d \sigma}{d p_{T}^{2}}\left(\mathrm{mb} /\left((\mathrm{ccl} / \mathrm{r})^{2}\right)\right.$ (per nucleus) for $\pi^{0}$ production in $\pi^{-}$Be collisions at $530 \mathrm{GcV}, 0.25<y_{\mathrm{cm}}<0.75$

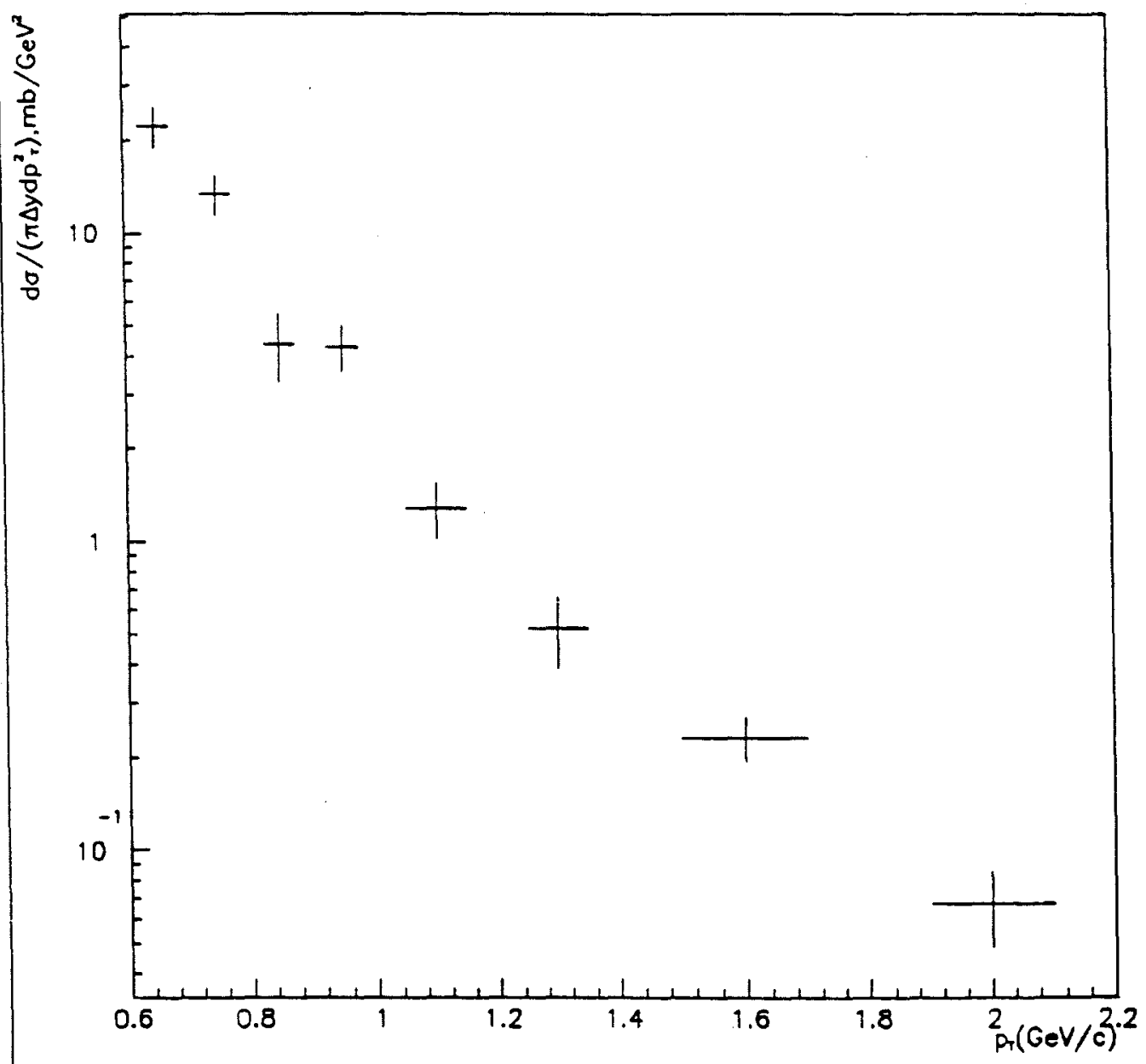


Figure 6.3: $\frac{1}{\pi \Delta y} \frac{d \sigma}{d p_{T}^{2}}\left(\mathrm{mb} /\left((\mathrm{Gcl} / \mathrm{c})^{2}\right)\right.$ (per nucleus) for $\pi^{0}$ production in $\pi^{-} A l$ collisions at $530 \mathrm{GeV},-0.25<y_{\mathrm{cm}}<0.25$

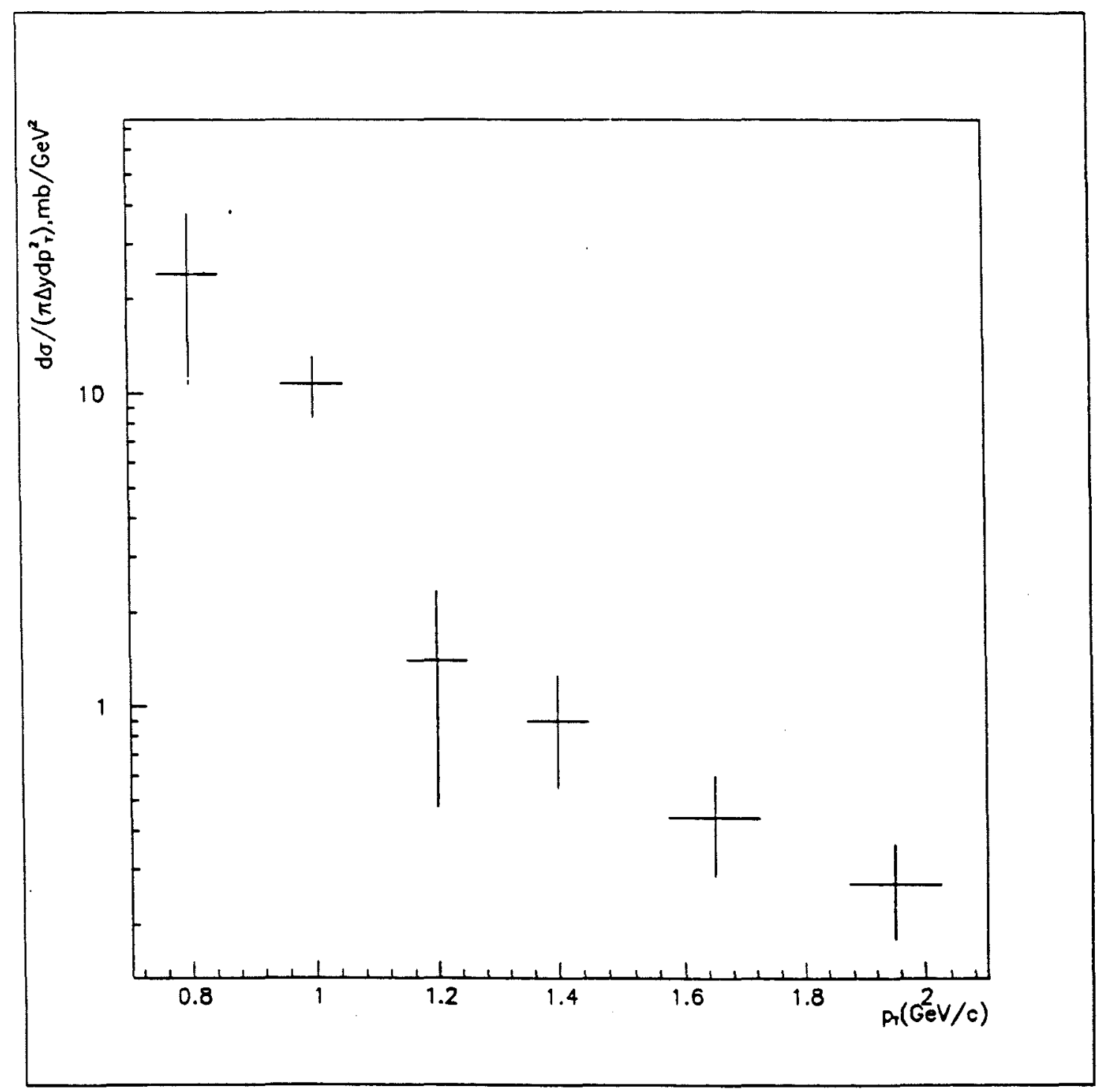


Figure 6.4: $\frac{1}{\pi \Delta y} \frac{d \sigma}{d p_{T}^{2}}\left(\mathrm{mb} /(G e \mathrm{l} / \mathrm{r})^{2}\right)$ (per nucleus) for $\pi^{0}$ production in $\pi^{-} A l$ collisions at $530 \mathrm{GeV}, 0.25<y_{\mathrm{cm}}<0.75$

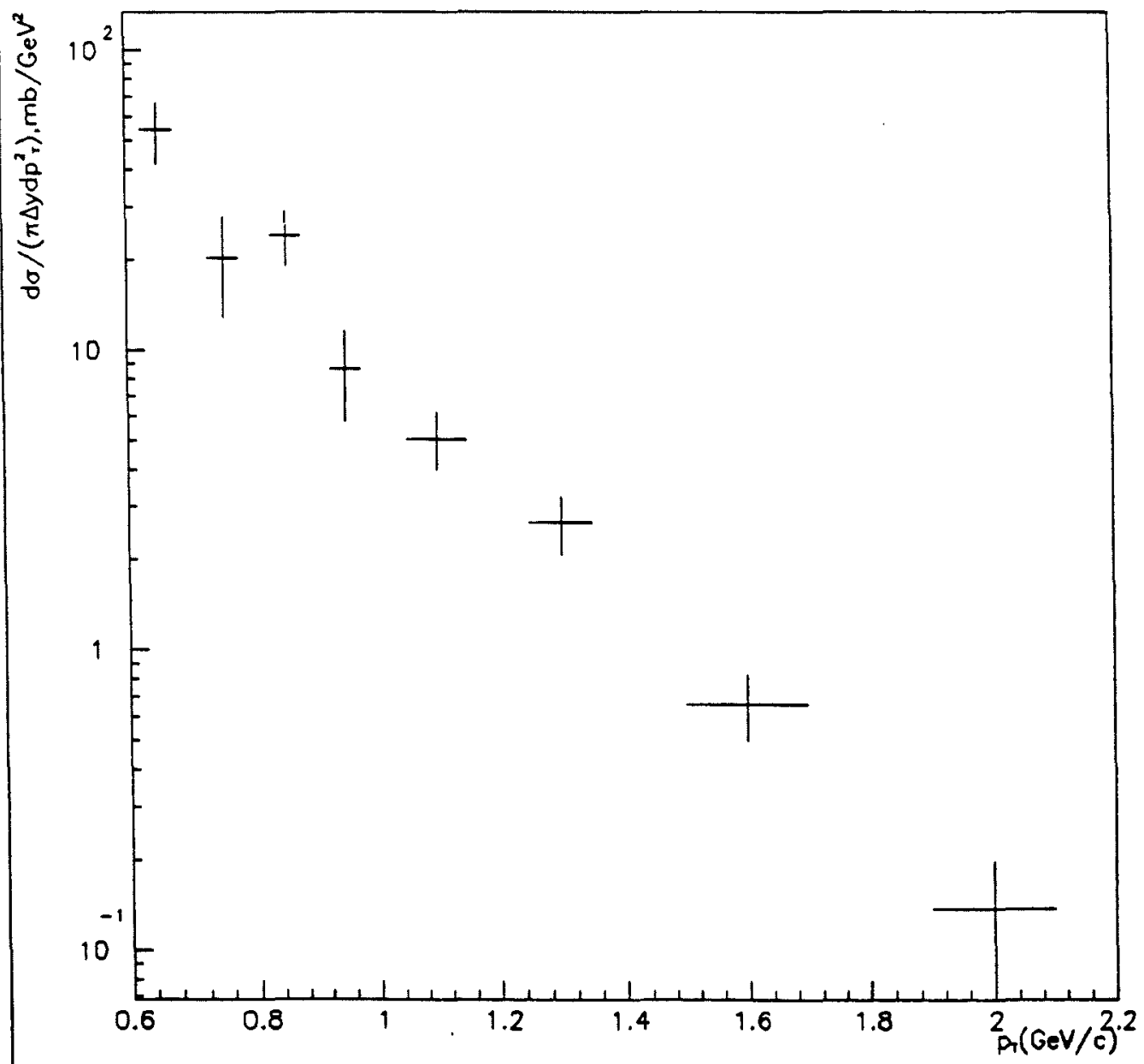




\subsection{Atomic dependence of inclusive hadronic cross sections}

The atomic dependence of the cross sections was determined in the same rapidity interval used to measure the inclusive cross sections presented in the previous section. It was parametrized in terms of the power $\alpha$ (see Sect [1.6]) as a function of $p_{T}$. The results are shown in:

1. Figure [6.5] and Table [6.3] for the central region $\left(-0.25<y_{c m}<0.25\right)$

2. Figure [6.6] and Table [6.4] for the forward region $\left(0.25<y_{c m}<0.75\right)$.

The atomic dependence of the cross section is usually less affected by systematic errors than the absolute cross section because because most systematic errors that may affect the determination of the latter cancel out when ratios are taken. In particular any overall normalization errors disappear if they are the same in both data samples; systematic errors in the calculation of the acceptance also vanish if the rapidity dependence of the production mechanism is the same for both targets in the region of interest. The latter assumption was validated in our study by establishing that the values of the power $\alpha\left(p_{T}\right)$ with and without acceptance corrections are compatible. The results presented here were obtained without applying acceptance corrections, which also results in a smaller statistical error, since in this case there is no contribution from the variations of the event weights in each $p_{T}$ bin.

The results for the atomic dependence of the cross sections will be discussed further in Sect. [6.6].

\subsection{Sources of systematic error}

In this section the sources of systematic errors for our measurement will be discussed. Since in general the distribution of the systematic errors is not known, in the following we quote the total range of systematic uncertainty, not the variance as it was done for the statistical errors. The principal source of systematic error in the measurement of the inclusive cross sections is the uncertainty in the $p_{T}$ scale, which 
Figure 6.6: The value of the power $\alpha$ in . $1^{\prime \prime}$ for $\pi^{\prime \prime}$ production in collisions at 530 $\mathrm{GeV}, 0.25<y_{\mathrm{cm}}<0.75$

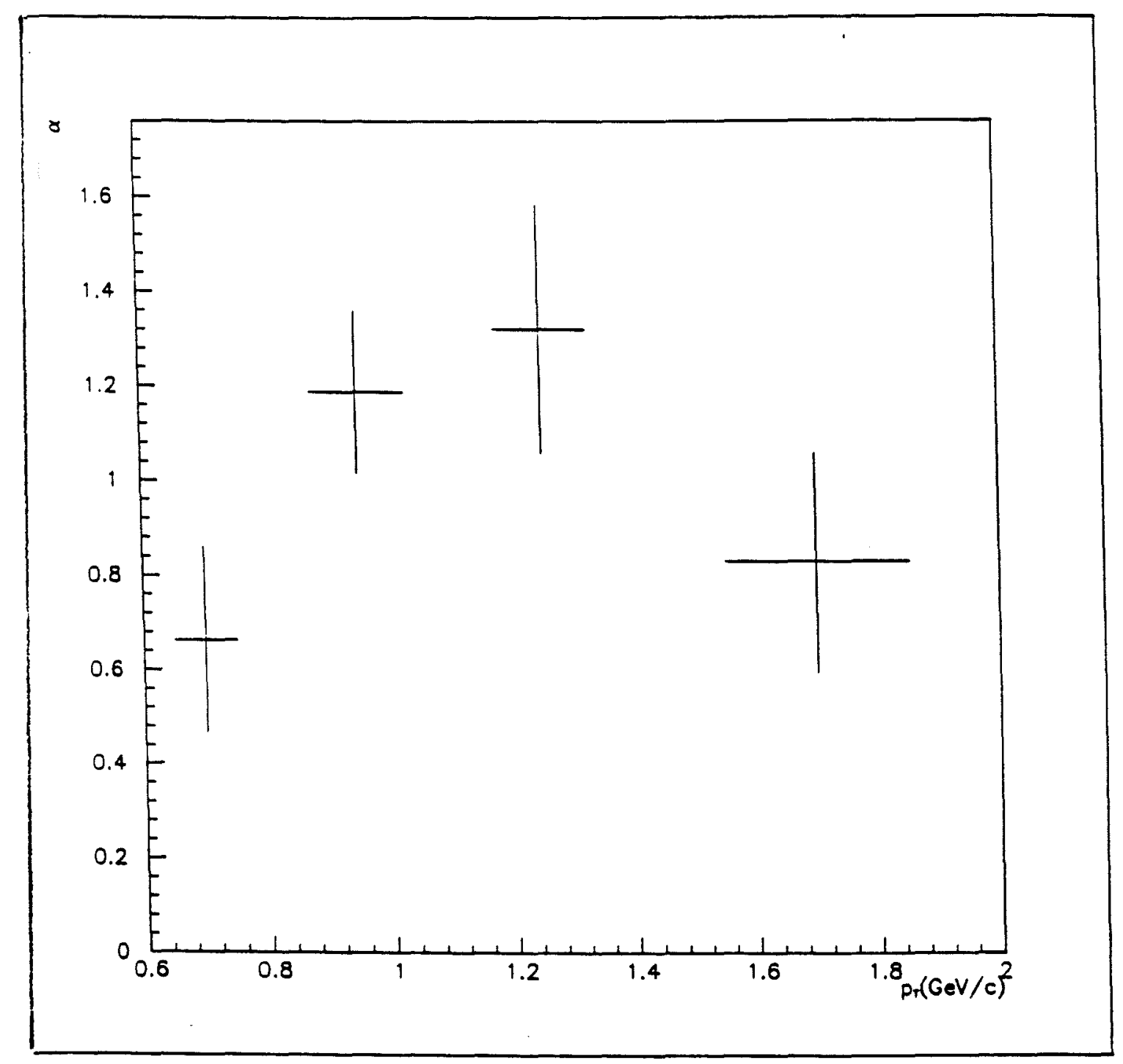


Table 6.3: The value of the power $\alpha$ in $A^{\alpha}$ for $\pi^{0}$ production in collisions at 530 $\mathrm{GeV},-0.25<y_{\mathrm{cm}}<0.25$

\begin{tabular}{|l|c|}
\hline$p_{T}(\mathrm{GeV} / \mathrm{c})$ & $\alpha$ \\
\hline $0.6-1.0$ & $0.89 \pm 0.21$ \\
\hline $1.0-1.5$ & $0.70 \pm 0.25$ \\
\hline $1.5-2.0$ & $1.25 \pm 0.33$ \\
\hline
\end{tabular}

Table 6.4: The value of the power $\alpha$ in $A^{\alpha}$ for $\pi^{0}$ production in collisions at 530 $\mathrm{GeV}, 0.25<y_{\mathrm{cm}}<0.75$

\begin{tabular}{|l|c|}
\hline$p_{T}(\mathrm{GeV} / \mathrm{c})$ & $\alpha$ \\
\hline $0.6-0.8$ & $0.66 \pm 0.20$ \\
\hline $0.8-1.1$ & $1.19 \pm 0.17$ \\
\hline $1.1-1.4$ & $1.32 \pm 0.26$ \\
\hline $1.4-2$. & $0.83 \pm 0.25$ \\
\hline
\end{tabular}


is caused mainly by uncertainty in the tail correction to the energy measurement. A small shift in $p_{T}$ causes a large uncertainty in the absolute normalization because of the steep fall-off of the $p_{T}$ spectrum. The $p_{T}$ of a particle, with high energy $\mathrm{E}$, at an angle $\theta$ with respect to the beam axis, is essentially $E \sin (\theta)$. The uncertainty in the $p_{T}$ scale can be written as:

$$
\left(\Delta p_{T} / p_{T}\right)^{2}=(\Delta E / E)^{2}+(\Delta \sin \theta / \sin \theta)^{2}
$$

Since the position resolution in the EMLAC is better than $1 \mathrm{~mm}$, the contribution due to the second term in the above equation is negligible.

The inclusive cross sections can be parametrized using the following function (see Sect. [1.5])

$$
d \sigma / d p_{T}^{2}=\frac{C}{\left(p_{T}^{2}+\delta^{2}\right)^{n}}
$$

For the purpose of these considerations we assume $\delta=0.64(\mathrm{GeV} / \mathrm{c})$ and $n=4$ (the detailed fit of our data to expression (6.2) is given in the next section). An error $\Delta\left(p_{T}\right)$ will cause a relative error in the cross section:

$$
\frac{\Delta\left(d \sigma / d p_{T}^{2}\right)}{d \sigma / d p_{T}^{2}}=\frac{2 n p_{T}}{p_{T}^{2}+\delta^{2}} \Delta\left(p_{T}\right)
$$

It is usually difficult to estimate the uncertainty in the energy scale. E706 will determine that uncertainty mainly from a comparision of the positions of the $\pi^{0}$ and of the $\eta^{0}$ in the $\gamma \gamma$ mass spectrum at large $p_{T}$. The main source of uncertainty at large $p_{T}$ is the error in the determination of the gain corrections. Our measurement is at low $p_{T}$ and our main source of uncertainty comes from additive effects (uncertainties in the pedestal subtraction and in the tail correction).

The systematic error in our energy scale was estimated by studying the variation of the $\pi^{0}$ mas in the phase space available for our measurement. This was first done using the sample of Monte Carlo events described in Sect [5.4]. The position of the $\pi^{0}$ peak varied by about $3 \mathrm{MeV} / \mathrm{c}^{2}$ in the $p_{T}$ range between 0.7 and $2.0 \mathrm{GeV} / \mathrm{c}$; it varied by not more than $4 \mathrm{MeV} / \mathrm{c}^{2}$ in the energy range of $12-32 \mathrm{GeV}$.

A similar study was also performed on the Be data; unfortunately the statistics did not permit an investigation of more than 3 regions in energy or $p_{T}$. The typical 
variations were not more than 5 or $6 \mathrm{MeV} / \mathrm{c}^{2}$. From the above considerations our estimate for the systematic variation of the $p_{T}$ scale over our $p_{T}$ range is $\approx 5 \%$, which gives a systematic error in the inclusive cross section of about $31 \%$ at $p_{T}=0.6$ $\mathrm{GeV} / \mathrm{c}$ and of $18 \%$ at $p_{T}=2 \mathrm{GeV} / \mathrm{c}$.

The systematic error due to the background subtraction was estimated by fixing the shape of the term representing the background (see Sect [4.5]) and calculating the variation in the normalization constant corresponding to a change of 1 in the $\chi^{2}$ for the fit. The errors obtained that way were $2.5 \%$ for $\Lambda \mathrm{l}$ and $1.8 \%$ for $\mathrm{Al}$; it may be noted however that this method may underestimate the error, because it assumes that the shape of the background is fixed.

An upper limit to the overall normalization error coming from the determination of the incident beam can be inferred from the considerations given in Sect. [4.7] and it is about $10 \%$.

A preliminary version of EMIREC was used in the analysis for this thesis. Since then, the program has been improved substantially for the purpose of reconstructing high energy showers; the splitting algorithm in particular has undergone extensive changes. Since the typical photon separation for the low $p_{T} \pi^{0}$ 's studied here is of the order of $10 \mathrm{~cm}$ in the FMI $\Lambda \mathrm{C}$, the two showers are well separated and a sophisticated splitting algorithm docs not improve the reconstruction. The reconst,ruction algorithm used was suitable for the data, and limited primarily by the correction for tails of showers, as indicaled above.

\subsection{Conclusions}

The measurement of $\pi^{0}$ production at low transverse momentum presented in this thesis is the first determination for nuclear targets at energies above $200 \mathrm{GeV}$. It is indeed far more difficult from the experimental point of view to measure the yicld of neutral pions than the yicld of charged pions. Since E706 was designed for the study of high $p_{T}$ photons, the acceptance for low $p_{T} \pi^{0}$ was rather restricted, especially given the fact that the $L A C$ is not sensitive to very low energy showers. We managed nevertheless to mcasure the inclusive yield of $\pi^{0}$ 's and our results can be used for testing models of particle production and in gauging backgrounds for other experiments at high energy. Although our determination is limited by 
Table 6.5: The value of the power $\alpha$ in $A^{\alpha}$ for $\pi^{0}$ production in collisions at 530 $\mathrm{GeV}$ as a function of $p_{T},-0.25<y_{\mathrm{cm}}<0.75$

\begin{tabular}{|l|c|}
\hline$p_{T}(\mathrm{GeV} / \mathrm{c})$ & $\alpha$ \\
\hline $0.6-1.0$ & $0.86 \pm 0.12$ \\
\hline $1.0-2.1$ & $1.02 \pm 0.13$ \\
\hline
\end{tabular}

statistics, it is not affected by uncertainties associated with large backgrounds; as a matter of fact the ratio of the signal to the background is at the least two times more favorable than for the NA27 data described in Sect. [1.5].

We will first discuss the implications of our measurement of the atomic dependence of the $\pi^{0}$ cross section, then we will draw our conclusions from the measurement of the inclusive cross sections.

The average value of $\alpha$ in the phase space of $p_{T}$ and rapidity available for our measurement was determined to be $\alpha=0.904 \pm 0.097$. This value is larger by about two standard deviations than the value of $\alpha$ expected for the total inclastic cross section (see Sect [1.6]) and is quite close to the valuc $\alpha=1$ obtained if nuclcons behave as free particles. 'To investigate the $p_{T}$ dependence of $\alpha$, the data were divided into two $p_{T}$ regions, using the entire rapidity range, as shown in Table [6.5]. The values of $\alpha$ are consistent with an increase with $p_{T}$ and show that the hard scaltering regime is reached at the higher end of the $p_{T}$ scale. Although errors are large, this is in agreernent with previous measurements on charged pions [25].

The dependence of $\alpha$ on rapidity was also investigated by comparing the value of $\alpha$ in the central and forward rapidity region, integrating over the entire $p_{T}$ range $\left(0.7 \mathrm{GeV} / \mathrm{c}<p_{T}<2.1 \mathrm{GeV} / \mathrm{c}\right)$. Our results are presented in Table [6.6] and within errors do not exhibit any dependence on rapidity.

We determined the integrals of the inclusive difrerential cross section per nucleus given in Eq. (6.1) over the entire $p_{T}$ range of our data:

$$
\sigma=\int_{r_{T 1}}^{p_{T 2}} \frac{d p_{T}^{2}}{2} \int_{y_{1}}^{y_{2}} d y \int_{0}^{2 \pi} d \phi \frac{d \sigma\left(y, p_{T}^{2}\right)}{\pi d p_{T}^{2} d y}
$$

$\left(p_{T 1}, p_{T 2}, y_{1}\right.$ and $y_{2}$ are the lower and upper limits of the $p_{T}$ and $y$ ranges taken 
Table 6.6: The value of the power $\alpha$ in $A^{\alpha}$ for $\pi^{0}$ production in collisions at 530 $\mathrm{GeV}$ as a function of center of mass rapidity, $0.7 \mathrm{GeV} / \mathrm{c}<p_{T}<2.1 \mathrm{GeV} / \mathrm{c}$

\begin{tabular}{|l|c|}
\hline rapidity & $\alpha$ \\
\hline$-0.25<y_{c m}<0.25$ & $0.87 \pm 0.17$ \\
\hline$+0.25<y_{c m}<0.75$ & $0.92 \pm 0.12$ \\
\hline
\end{tabular}

Table 6.7: Integraled inclusive cross scctions (mbarns) per nucleus for $\pi^{0}$ production in $\pi^{-} B e$ and $\pi^{-} A l$ interactions at $530 \mathrm{GeV}$

\begin{tabular}{|l|c|c|c|}
\hline rapidity range & $p_{T}(\mathrm{GcV} / \mathrm{c})$ range & $\pi^{-} B e$ & $\pi^{-} A l$ \\
\hline$-0.25<y_{\mathrm{cm}}<0.25$ & $0.7<p_{T}<2.1$ & $10.2 \pm 1.4$ & $21.7 \pm 6.9$ \\
\hline$+0.25<y_{\mathrm{cm}}<0.75$ & $0.7<p_{T}<2.1$ & $7.5 \pm 0.6$ & $21.1 \pm 2.5$ \\
\hline$-0.25<y_{\mathrm{cm}}<0.75$ & $0.7<p_{T}<2.1$ & $17.7 \pm 1.5$ & $42.8 \pm 7.2$ \\
\hline$-0.25<y_{\mathrm{cm}}<0.75$ & $0.6<p_{T}<2.1$ & $27.2 \pm 2.5$ & $66.3 \pm 10.7$ \\
\hline$+0.25<y_{\mathrm{cm}}<0.75$ & $0.6<p_{T}<2.1$ & $12.1 \pm 0.9$ & $32.0 \pm 3.5$ \\
\hline
\end{tabular}

into consideration) The results are given in Table [6.7]. It is interesting to note the rapidity dependence of the cross sections: the values of the cross sections in the forward and in the central regions are comparable, unlike in the case of incident protons, where the cross sections is much larger in the central region [10]. This can be qualitatively understood by taking into account the quark structure [56] of the projectile and the target. Since in the center of mass reference frame a $\pi^{-}$and nucleon have equal mornentum, but the incident $\pi^{-}$consists of two valence quarks, whereas the struck nucleon consists of three valence quarks, the average fraction of momentum carried by the quarks forming the $\pi^{-}$is larger. The cross section for particle production is therefore peaked at positive rapidities.

Our data for the inclusive cross section were fitted to the same form (6.2), used by experiment NA27 at CERN (sec Scct. [1.5], where the NA27 results are presented in detail). After a preliminary fit, the normalization parameters (to which 
Table 6.8: Values of the shape parameters in the fits to the inclusive cross sections for $\pi^{0}$ production in collisions at $530 \mathrm{GeV},-0.25<y_{\mathrm{cm}}<0.75$

\begin{tabular}{|l|c|c|c|}
\hline Target & $\delta(G e V / c)$ & $n$ & $\chi^{2}$ per degree of frcedom \\
\hline $\mathrm{Be}$ & $0.63 \pm 0.15$ & $4.22 \pm 0.12$ & $16 / 6$ \\
\hline $\mathrm{Al}$ & $0.69 \pm 0.27$ & $4.09 \pm 0.16$ & $2.2 / 6$ \\
\hline
\end{tabular}

the fit is not very sensitive) were determined. $\Lambda$ final fit was performed keeping the normalization parameters fixed in order to determine the shape parameters with a smaller statistical error. The central and forward rapidity regions were combined in the fits; separate fits were also carried out and agreed with the combined fit, thus indicaling that the $p_{T}$ dependence of the cross sections in both rapidity regions is the same within errors. The results of the fits are presented in Table [6.8], Fig. [6.7] and Fig [6.8]. The functional form that was chosen represents the data satisfactorily (the relatively high $\chi^{2}$ value obtained for the fit to the Be cross section corresponds to fuctuations about the fit of only tivo data points)

'The hypothesis of scaling introduced in Sect. [1.1], was checked by comparing our measurement of the cross section with the N 127 results. If only the $p_{T}$ dependence of the cross section is taken into account, the values of the shape parameters $n$ and $\delta$ given in Table [6.8] are in substantial agreenent with the values derived by NA27 $(\delta=0.64 \pm 0.02(\mathrm{GoV} / \mathrm{c}$ and $n=4.03 \pm 0.07)$, thus showing the validity of the scaling hypothesis for the $p_{T}$ dependence. The agrcement of the normalizations was also verified by scaling the $\mathrm{N} \wedge 27$ results on hydrogen to our targets, by using the average value of $\alpha$ given at the beginning of this section. At small $p_{T}$ our cross sections agree within errors with the NA27 results. Our results are not sensitive to small deviations from the $A^{\alpha}$ parametrization, which have been observed in $\pi^{+} / \pi^{-}$ production on hydrogen targets [25]. There is no evidence of scaling violations of the invariant cross section belween our cnergy and the energy of the NA27 data, once the $\Lambda$-dependence of the data is taken into account.

The constant $\delta$ is essential for the description of the inclusive cross section distribution up to $p_{T}$ values comparable with $\delta$, whereas in the high $p_{T}$ limit the shape of the distribution is determined by the $\left(\frac{1}{p_{T}^{2}}\right)^{n}$ term. The differential cross 
Figure 6.7: Fit to $\frac{1}{\pi \Delta y} \frac{d \sigma}{d p_{T}^{2}}$ (per nucleus) (mb/( $\left.\left.\mathrm{GeV} / \mathrm{c}\right)^{2}\right)$ for $\pi^{0}$ production in $\pi^{-} B e$ collisions at $530 \mathrm{GeV},-0.25<y_{\mathrm{cm}}<0.75$ (the dashed lines represent the values of the fit)

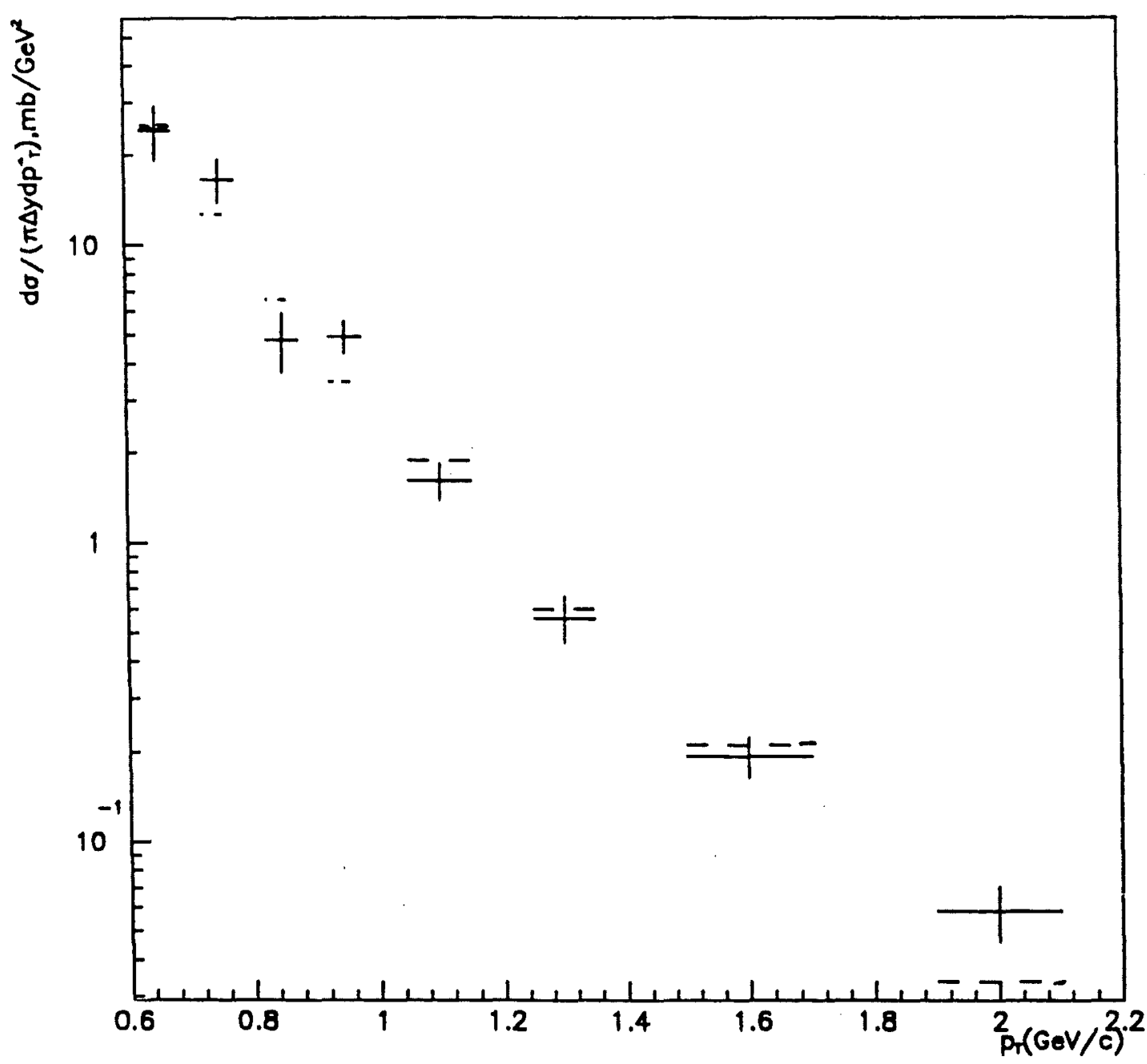


Figure 6.8: Fit to $\frac{1}{\pi \Delta y} \frac{d \sigma}{d p_{T}^{2}}$ (per nucleus) $\left(\mathrm{mb} /(G e V / c)^{2}\right)$ for $\pi^{0}$ production in $\pi^{-} A l$ collisions at $530 \mathrm{GeV},-0.25<y_{c m}<0.75$ (the dashed lines represent the values of the fit)

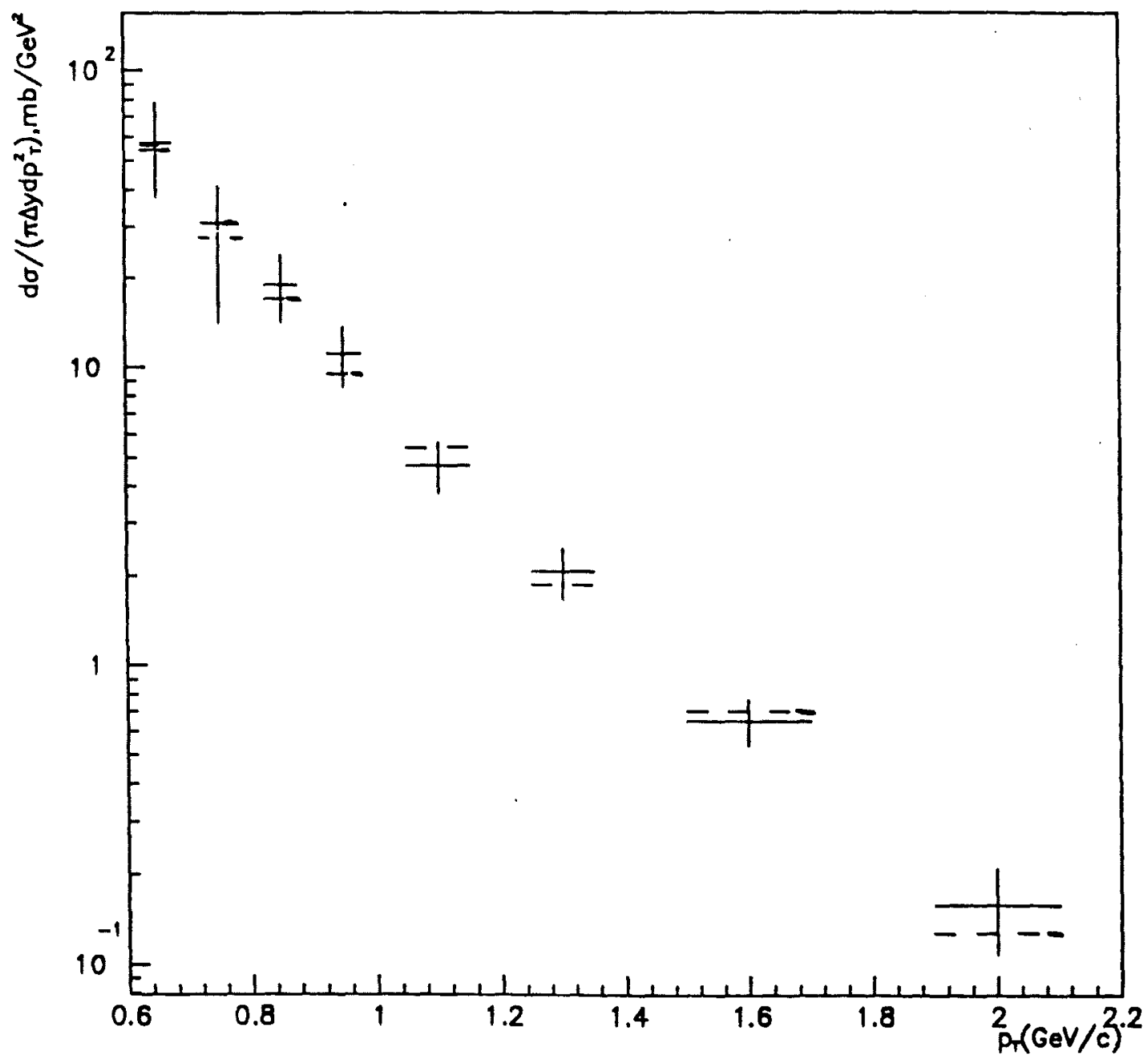


sections at the higher end of the $p_{T}$ scale show a clear departure from exponential behavior, which is commonly used to parametrize the data in the very soft region. In fact, the highest $p_{T}$ data point in $\mathrm{Be}$, which contributes to the large $\chi^{2}$ in Table [6.8], may be indicative of the onset of scale violations at largest $p_{T}$. Our value for $n$ is in agreement with the prediction of the Constituent Interchange Model (CIM) $[57,58,59]$, which gives a $2 n=8$, assuming that the interacting objects are $q \bar{q}$ bound systems (pions). In perturbative QCD (see Sect. [1.2]) hadrons behave as composite particles, made of quasi-free point-like constituents (partons). Parton-parton scattering enhances dramatically the inclusive particle yields at high $p_{T}$ [60]. If all masses are neglected and partons are considered entirely free (naive parton model), the elementary parton cross section is scale-free and it exhibits a $p_{T}^{-4}$ dependence at high $p_{T}[61]$. Our value of $n$ can be interpreted as an indication that at the higher end of our $p_{T}$ scale the production process is influenced only by the onset of perturbative QCD and parton-parton scattering is still obscured by other processes whose relevance should decrease at truly asymptotic energies and $p_{T}$ values. 


\section{Bibliography}

[1] H. Yukawa, Proc. Math. Soc. Japan 17 (1935) 48

[2] Perturbative Quantum Chromodynamics, Physics Reports Reprint Book Series Vol. 5 , ed. M. Jacob, North Holland (1982)

[3] K. Fialkowski and W. Kittel, Rep. Prog. Phys. 46 (1983) 1283

[4] W. Ochs, Nucl. Phys. B 118 (1977) 397

[5] B. Anderson, et al., Phys. Lett. 71B (1977) 337

[6] H. Boggild and T. Ferbel, in Annual Review of Nuclear Science (Palo Alto Series), (1974) 451. In section 1.3 and 1.4 we follow the exposition provided in this reference.

[7] A. A. Logunov et al., Phys. Lett. B25 (1967) 611

[8] R. P. Feynman, Phys. Rev. Lett 23 (1969) 1415

[9] J. Benecke et al., Phys. Rev. D5 (1969) 2159

[10] I. V. Ajinenko et al., Z. Phys. C35 (1987) 7

[11] M. Aguilar-Benitez et al., Z. Phys. C34 (1987) 419

[12] J. Bartke et al., Acta Phys. Pol. B5 (1984) 689

[13] I. V. Ajinenko et al., Nucl. Phys. B162 (1980) 61

[14] M. Barth et al., Z. Phys. C22 (1984) 23 
[15] J. Guy et al., Nucl. Phys. B155 (1979) 320

[16] J. Elliot et al., Nucl. Phys. B133 (1978) 1

[17] K. Boeckman et al., Nucl. Phys. B119 (1977) 253

[18] N. N. Biswas et al., Phys. Rev. D10 (1974) 3579

[19] I. V. Ajinenko et al, Sov. J. Nucl. Phys 31 (1980) 62

[20] M. Alston-Garnjost et al, Phys. Rev. Lett. 35 (1975) 142

[21] A. V. Barnes et al, Nucl. Phys. B145 (1978) 45

[22] R. G. Kennet et al, Nucl. Phys. B177 (1981) 1

[23] B. Durand and J. Krebs, Phys. Rev. D21 (1980) 3137

[24] R. J. Glauber, in Lectures in Theoretical Physics, Vol. 1, ed. W. E. Brittin and L. E. Dunam, Inter-Science, New York, (1959)

[25] D. Antreasyan et al., Phys. Rev. D 19 (1979) 764

[26] J. W Cronin et al., Phys. Rev. D 11 (1975) 3105

[27] L. Kluberg et al., Phys. Rev. Lett. 38 (1977) 670

[28] K. Gottfried, Phys. Rev. Lett. 32 (1974) 957

[29] G. Farrar, Phys. Lett. 56B (1975) 185

[30] J. Pumplin and E. Yen, Phys. Rev. D11 (1975) 1812

[31] A. Krzywicki et al., Phys. Lett. 85B (1979) 407

[32] A. Capella and J. Tran Thanh Van, Phys. Lett. 93B (1980) 146

[33] W. Baker, E706 Note 65 (1983), unpublished

[34] S. Mani, Ph. D thesis, University of Pittsburg (1986), unpublished

[35] E. Engels et al.,NIM A253 (1987) 523 
[36] E. Engels, et al., XXIV International Conference on Iigh Energy Physics, Munich, Federal Republic of Germany, August 4-10, 1988

[37] C. Bromberg, E706 Note 094 (1984), unpublished

[38] F. Lobkowicz et al., NIM, 4235 (1985) 332. In Section 2.5 we follow the exposition provided in this reference.

[39] J. Huston, et al., XXIV International Conference on High Energy Physics, Munich, Federal Republic of Germany, August 4-10,1988

[40] M. Glaubman, E706 Note 88 (1984), unpublished

[41] C. Brassard, NIM 162 (1979) 29

[42] W. Willis and V. Radeka, NIM 162 (1974) 221

[43] T.F. Droedge, K.J. Turner and T.K. Ohska, Fermilab Note PIN 52H (1982), unpublished

[44] G. Drake et al, IEEE Nuclear Science Symposium, San Francisco, California (1985)

[45] K. J. Turner et al., Fermilab Note PIN 7 ZA (1983), unpublished

[46] T.F. Droedge, F. Lobkowicz and F. Fukushima, IEEE Trans. Nucl. Sci. NS-25 (1978). This reference describes a system conceptually identical to the LAC amplifier.

[47] E. Prebys, Qualifying Brief, University of Rochester (1986), unpublished

[48] P. Lukens, K. Ruddick E706 Note 141 (1985), unpublished

[49] Fermilab VAX Online Note PN 252.2 (1986), unpublished, and references therein.

[50] H. J. Klein and J. Zoll, PATCHY REFERENCE MANUAL, CERN PROGRAM LIBRARY, CERN (1983), unpublished

[51] R. Brun, M. Goossens and J. Zoll, ZEBRA, CERN PROGRAM LIBRARY Q100, CERN (1985), unpublished 
[52] W. Nelson, II. Hirayama and D. Rogers, SLAC-Report-265 (1985), unpublished

[53] R. Brun, F. Bruyant and $\Lambda$. McPherson, GEANT3, CERN DATA HANDLING DIVISION, DD/US/86 (1986), unpublished, and references therein.

[54] R. L. Walker, CIT Internal Report 59 (1971), unpublished

[55] F. Paige and P. Protopopescu, in Snowmass Supercollider Workshop, R. Donaldson ed., Snowmass (1982)

[56] K. Gottried and V. Weisskopf, in Concepts of Particle Physics, Oxford University Press (1986)

[57] R. Blankenbecker and S.J. Brodsky, Phys. Rev. D10 (1974) 2973

[58] R. Blankenbecker and S.J. Brodsky, Phys. Rev. D12 (1974) 3469

[59] R. Blankenbecker, S.J. Brodsky and D. Sivers, Phys. Rep. 23C (1976) 1

[60] G. Altarelli, Phys. Rep. 81 (1982) 1

[61] S. M. Berman, J. D. Bjorken and J. Kogut, Phys. Rev. D4 (1971) 3388 